2017-01

\title{
Guidelines for Family-Centered Care in the Neonatal, Pediatric, and Adult ICU.
}

\author{
Davidson, JE
}

http://hdl.handle.net/10026.1/13043

10.1097/CCM.0000000000002169

Crit Care Med

All content in PEARL is protected by copyright law. Author manuscripts are made available in accordance with publisher policies. Please cite only the published version using the details provided on the item record or document. In the absence of an open licence (e.g. Creative Commons), permissions for further reuse of content should be sought from the publisher or author. 
Davidson J. et al 2017. Guidelines for Family-Centered Care in the Neonatal, Pediatric and Adult Intensive Care Unit Critical Care Medicine. 45(8):1352-1358, AUG 2017

Author copy. Accepted for publication $7^{\text {th }}$ September 2016.

DOI: $10.1097 / C C M .0000000000002523$

Title: Guidelines for Family-Centered Care in the Neonatal, Pediatric and Adult Intensive Care Unit

\section{Corresponding Author:}

Judy E. Davidson DNP RN FCCM FAAN, jdavidson@ucsd.edu

\section{Authors: (No authors have conflicts to report)}

Judy E. Davidson DNP RN FCCM FAAN, University of California San Diego Health, San Diego, California.

Rebecca A. Aslakson MD PhD FAAHPM, Department of Anesthesiology and Critical Care Medicine; Department of Oncology and the Palliative Care Program in the Sidney Kimmel Comprehensive Cancer Center at Johns Hopkins; Department of Health, Behavior, and Society in the Johns Hopkins Bloomberg School of Public Health; The Johns Hopkins Hospital, Baltimore, Maryland.

Ann C. Long MD MS, Cambia Palliative Care Center of Excellence, Division of Pulmonary and Critical Care Medicine, Harborview Medical Center, University of Washington, Seattle, Washington.

Kathleen A. Puntillo PhD RN FAAN FCCM, Department of Physiological Nursing, University of California, San Francisco.

Erin K. Kross MD Cambia Palliative Care Center of Excellence, Division of Pulmonary and Critical Care Medicine, Harborview Medical Center, University of Washington, Seattle, Washington.

Joanna Hart MD MS Division of Pulmonary, Allergy, and Critical Care Medicine, Fostering Improvement in End-of-Life Decision Science Program, Center for Clinical Epidemiology and Biostatistics, Perelman School of Medicine, University of Pennsylvania, Philadelphia, Pennsylvania.

Christopher E. Cox MD MPH, Division of Pulmonary and Critical Care Medicine, Program to Support People and Enhance Recovery, Duke University, Durham, North Carolina.

Hannah Wunsch MD MSc, Department of Critical Care Medicine Sunnybrook Health Sciences Centre; Department of Anesthesia and Interdepartmental Division of Critical Care Medicine, University of Toronto, Ontario, Canada 
Mary A. Wickline MLIS, M.Ed. University of California, San Diego, California.

Mark E. Nunnally MD FCCM University of Chicago; Chicago, Illinois; Director Adult Critical Care Services, New York University NY.

Giora Netzer MD MSCE Division of Pulmonary and Critical Care Medicine, University of Maryland School of Medicine, Baltimore, MD.

Nancy Kentish-Barnes PhD Saint-Louis University Hospital, Paris, France.

Charles L. Sprung MD MCCM JD Hadassah Hebrew University Medical Center, Jerusalem, Israel.

Christiane Hartog MD Jena University Hospital, Department of Anaesthesiology and Intensive Care Medicine, Jena, Germany.

Professor Maureen Coombs PhD RN, Graduate School of Nursing Midwifery and Health, Victoria University of Wellington; Capital and Coast District Health Board, Wellington, New Zealand.

Rik T. Gerritsen MD FCCM Center of Intensive Care, Medisch Centrum, Leeuwarden, The Netherlands.

Ramona O. Hopkins PhD, Brigham Young University, Provo, Utah; Pulmonary and Critical Care Medicine, Intermountain Medical Center, Murray Utah.

Linda S. Franck PhD RN FRCPCH FAAN Department of Family Health Care Nursing, University of California, San Francisco.

Yoanna Skrobik MD FRCP(c), MSc McGill University, Montreal, Canada.

Alexander A. Kon MD FCCM, Naval Medical Center San Diego and University of California San Diego, San Diego, CA

Elizabeth A. Scruth PhD RN CCRN CNS FCCM Quality and Regulatory Services, Kaiser Permanente, Oakland CA.

Maurene A. Harvey MPH MCCM Critical Care Educator and Consultant, Lake Tahoe, Nevada.

Mithya Lewis-Newby MD MPH Seattle Childrens Hospital, Seattle, Washington.

Douglas B. White MD MAS, Program on Ethics and Decision Making in Critical Illness; Department of Critical Care Medicine, University of Pittsburgh medical Center, Pittsburgh, Pennsylvania.

Sandra M. Swoboda MS RN FCCM Johns Hopkins University Schools of Medicine and Nursing, Baltimore, Maryland.

Colin R. Cooke MD MS, University of Michigan Hospitals, North Campus Research Complex, Ann Arbor, Michigan.

Mitchell M. Levy MD MCCM FCCM, The Warren Alpert School at Brown University, Providence, Rhode Island.

Elie Azoulay MD PhD, Saint-Louis University Hospital, Paris, France. 
J. Randall Curtis MD MPH Cambia Palliative Care Center of Excellence, Harborview Medical Center, University of Washington, Seattle, Washington.

\section{Acknowledgements:}

Sarah A. Kraus MPH Society of Critical Care Medicine, Mt. Prospect, Illinois

Patricia G. Graham MS RN CCRN University of California, San Diego, San Diego, California

Scot Halpern MD, University of Pennsylvania School of Medicine, Division of Pulmonary and Critical Care Medicine, Philadelphia, Pennsylvania

Miachael Quasney MD, University of Michigan, Ann Arbor, Michigan

Kathleen M. Kelly MD FACS FCCM, Janssen Research and Development, Morristown, New Jersey

Dan R. Thompson MD MA MCCM FACP, Alden March Bioethics Institute, Albany, New York

David Y. Hwang MD, Assistant Professor of Neurology; Division of Neurocritical Care and Emergency Neurology; Yale School of Medicine; New Haven, Connecticut

Heather M. Bullard PharmD, BCCCP, Clinical Pharmacy Specialist, Cardiothoracic Surgery; Department of Pharmacy; The University of Chicago medicine; Chicago, Illinois

LeeAnn Christie MSN RN Dell Children's Medical Center of Central Trexas; Austin, Texas

Meg Frizzola DO, Division of Critical Care Medicine; Medical Director, Pediatric Intensive Care Unit; Assistant Professor of Pediatrics; Sidney Kimmel Medical College; Thomas Jefferson University, Philadelphia, Pennsylvania

Serena A. Harris PharmD, BCPS, BCCCP; Department of Pharmacy; Eskenazi health; Indianapolis, Indiana

Mathhew E. Lissauer MD, Surgical Critical Care; Rutgers-Robert Wood Johnson Medical School; New Brunswick, New Jersey

Appreciation is sent to the patients and family members who volunteered their time to validate the PICO questions and outcomes of interest.

Keywords:

"Family"[Mesh]

"Patient-Centered Care"[Mesh]

"Intensive Care"[Mesh]

"Intensive Care, Neonatal"[Mesh]

"Critical Care"[Mesh]

"Critical Care Nursing"[Mesh]

Family-centered care

Family Nursing 


\begin{abstract}
Objective: To provide clinicians with evidence-based strategies to optimize support of the family of critically ill patients in the ICU.

Methods: We used the Council of Medical Specialty Societies principles for the development of clinical guidelines as the framework for guideline development. We assembled an international multidisciplinary team of 29 members with expertise in guideline development, evidence analysis and family-centered care to revise the 2007 Clinical Practice Guidelines for Support of the Family in the Patient-Centered Intensive Care Unit. We conducted a scoping review of qualitative research that explored family-centered care in the ICU. Thematic analyses were conducted to support Population, Intervention, Comparison, Outcome (PICO) question development. Patients and families validated the importance of interventions and outcomes. We then conducted a systematic review using the Grading of Recommendations, Assessment, Development and Evaluations (GRADE) methodology to make recommendations for practice. Recommendations were subjected to electronic voting with pre-established voting thresholds. No industry funding was associated with guideline development.
\end{abstract}

Results: The scoping review yielded 683 qualitative studies; 228 were used for thematic analysis and PICO question development. The systematic review search yielded 4158 reports after de-duplication and 76 additional studies were added from alerts and hand searches; 238 studies met inclusion criteria. We made 23 recommendations from moderate, low and very low level of evidence on the topics of: communication with family members, family presence, family support, consultations and ICU team members, and operational and environmental issues. We provide recommendations for future research and work-tools to support translation of the recommendations into practice.

Conclusions: These guidelines identify the evidence base for best practices for family-centered care in the ICU. All recommendations were weak, highlighting the relative nascency of this field of research and the importance of future research to identify the most effective interventions to improve this important aspect of ICU care. 


\section{Introduction}

There is increasing evidence of the significant impact that critical illness has on family members of the critically ill. Stressful decision making often falls to family members because most patients in the intensive care unit (ICU) are too ill to participate in decision-making (1). Furthermore, family members bear a significant burden of caregiving to the more than $50 \%$ of critical illness survivors who have post-discharge disability $(2,3)$. Approximately one-quarter to half of family members of critically ill children or adults experience psychological symptoms, including acute stress, post-traumatic stress, generalized anxiety, and depression both during and after the critical illness of a loved one (4-6). The sum total of family exposure to critical illness may result in what has been termed "Post-Intensive Care Syndrome-Family" (PICS-F) (3, 5). There is increasing awareness of the importance of improving outcomes for family caregivers and that support for family caregivers can also improve patient outcomes $(6,7)$.

Structured interventions and approaches to support family members of critically ill patients are needed both to mitigate the impact of the crisis of critical illness and to prepare family members for decision-making and caregiving demands. Family-centered care recognizes the central importance of the family to a patient's recovery and describes the responsibilities of the healthcare team to provide support for families of seriously ill patients. The objective of these guidelines is to provide clinicians with evidence-based strategies to optimize support of the family of critically ill patients in the ICU.

The original clinical practice guidelines for support of the family in the patient-centered ICU from the American College of Critical Care Medicine were published in 2007 (8). These prior guidelines have been one of the most widely cited documents on family-centered care and stimulated research on the role of families in ICU care. However, the prior guidelines were developed using what is now an outdated evidence analysis. The new guidelines described in this work are not an update of the prior guidelines, but instead are the result of a completely new and more rigorous analysis. These new guidelines represent the current state of international science in family-centered care and family support for family members of critically ill patients across the lifespan. We report here the process for the systematic review of the literature, grading of the evidence, consultation with patients and families, and development of consensus among international experts on the final recommendations herein.

\section{Methods}

These guidelines were developed using the Council of Medical Specialty Societies (CMSS) Principles for the Development of Specialty Society Clinical Guidelines framework (9). The guideline writing group was composed of international experts in the fields of neonatal, pediatric and adult critical and intensive care medicine and family-centered care. The goal was to create a document to optimize family-centered care for the global community of ICU clinicians who care for patients and family members of all age groups.

A strict conflict of interest process was followed according to SCCM procedures. A conflict of interest form was completed at the start of the process and yearly. Members were asked to report new conflicts at each meeting. Of the writing members, 19 out of 21 were chosen for their expertise on the topic of family-centered care. This was considered essential to product development and not considered a conflict. Authors did not review their own papers during evidence analysis and Grading of Recommendations, Assessment, Development and Evaluations (GRADE) scoring or write summaries of their own work. Authors with conflicts were asked to abstain from voting. By group consensus, authors were permitted to vote on the recommendations made partially as a result of their scientific contributions. In no circumstance 
did these votes make a difference in whether or not the recommendation was accepted

(Electronic Supplement Table 3: Voting Results). No authors had influence over the acceptance of the document. None reported the potential for financial gain that could cause bias. There was no industry involvement in the development of these Guidelines."

A scoping review was undertaken of all qualitative research that explored familycentered care in the ICU from the perspective of patients, families and clinicians. Thematic analyses of results were then used to develop clinical questions regarding best methods of providing family-centered care. In evidence-based practice the Population, Intervention, Comparator, Outcome (PICO) format of constructing a clinical question was used to focus the search. These questions were then used to locate results from quantitative studies testing interventions in specific areas of family-centered care in the ICU to improve outcomes of interest. These studies were reviewed and analyzed using the GRADE methodology to make recommendations for clinical practice.

\section{Search Methodology}

The perspectives of patients and family members were incorporated in guideline development in two ways: through literature review and direct consultation. We conducted a scoping review of the qualitative literature relevant to neonatal, pediatric and adult patient and family perspectives using PubMed, CINAHL, Web of Science, and PsycINFO. The purpose of this review was to identify issues of importance to patients and families to generate PICO questions. We conducted a broad general search of Web of Science on the topic "familycentered" OR "family-centred" to determine when family-centered care began to be commonly addressed in ICU studies. A citation report showed that the number of publications exceeded 100 per year beginning in 1994 [Electronic supplement Figure 1]. We used this as a basis to determine the year to begin our literature search (1994- ). Qualitative filtering of English language texts was adapted from McMaster's University Health Information Research Unit filters to identify non-experimental research $(10,11)$. We performed a PubMed MEDLINE search using this logic statement [see appendix 1: Complete search strategies]:

("Intensive Care Units"[Mesh] OR "Critical Care"[Mesh] OR "Critical Care

Nursing"[Mesh] OR "intensive care" OR "critical care")

AND

("family centered" OR "family centred")

AND

(interview*[Title/Abstract] OR interviews[MeSH:noexp] OR experience*[Text Word] OR qualitative[Title/Abstract])

Separate searches focused on family versus clinician perspectives and retrieved a total of 683 studies. Duplicates, single case studies, narrative reviews of the literature, and off-topic abstracts were eliminated, resulting in 228 final studies for the scoping review. These abstracts were then sorted into RefWorks ${ }^{\circledR}$ groups based on primary perspectives: studies describing patient/family perspectives $(n=133)$, or studies describing clinician perspectives $(n=118)$. Some studies overlapped both categories and evaluated family-centered care from multiple perspectives of clinicians, patients and family members $(n=23)$. Systematic, Cochrane, and narrative reviews were included $(n=12)$.

The guidelines writing group reviewed the abstracts for relevant findings. A master spreadsheet of study results was constructed to perform a thematic analysis from the results of each abstract. The themes were then clustered further into patient/family and clinician domains, which were then used to develop PICO questions (Electronic Supplement Table 1).

Former ICU patients and family members were recruited from the University of Maryland (UOM) School of Medicine and the University of California San Diego Health System (UCSD), 
as well as through patient advocacy organizations, including the ARDS Foundation, Survivors of Sepsis, and Project Help. Participants were also recruited by word of mouth by writing group members. This recruitment was done in parallel with an ongoing research study at the UOM (Institutional Review Board (IRB) HP-0058018), and UCSD (IRB 140458).

Patient and family participants $(n=27)$ were consulted at 3 time points during the guidelines preparation: 1 ) development of the definition of family and family-centered care; 2 ) creation of the domains to be considered for development of PICO questions; and 3) ranking of the importance of outcomes within the PICO questions. At each time point, interviews were scheduled by telephone or email at the preference of the participant. The scripts were developed by one committee member (JD) and validated for clarity by at least two guidelines writing group members. A committee member (GN or $P G)$ sent the script over email and read the script and associated questions during phone interviews.

\section{Definitions of "family" and "family-centered care"}

To ensure consensus and consistency in use of key concepts used in guidelines development, we identified previous published definitions of "family" and "family-centered care" from guidelines and key documents on family-centered care, and then conducted an online survey of the entire guidelines writing group to select definitions of family and family-centered. Full details of this process are described in Online Appendix A. We intentionally did not search patient or person-centered care because the focus of these Guidelines is on support of the family. However, we support the concept of person and patient-centered care. The proposed definitions were then reviewed and approved by a group of seven former ICU patients and family members. These final definitions for "family" and "family-centered care" were unanimously deemed appropriate and acceptable by the participating former ICU patients and family members. Our definitions are as follows:

Family is defined by the patient or, in the case of minors or those without decision making capacity, by their surrogates. In this context, the family may be related or unrelated to the patient. They are individuals who provide support and with whom the patient has a significant relationship.

Family-centered care is an approach to health care that is respectful of and responsive to individual families' needs and values.

\section{Defining the Relevant Outcomes and Prioritizing the Outcomes}

The guidelines writing group developed a list of domains for family-centered care outcomes through a review of the qualitative literature identifying domains important to patients and family members (12-23). We then had group members rate the relative importance of each outcome on a scale of extremely important (10) to not at all important (0). In addition, a sample of survivors and family members also rated the importance of these outcomes on the same scale producing similar results and supporting the rating results. These outcomes and their importance scores are shown in Electronic Supplement Table 2.

Following the rating of potential outcomes to be used in the PICO questions, patients and family members were again surveyed. The 24 outcomes were rated using a 0-10 numeric rating scale and then ranked according to their mean rating. Nine patients and family members participated. In general, patients and family members scored all outcomes higher than the writing group, with less discrimination between most and least important. No new outcomes were identified in the open comments section. Of interest, patients and family members scored clinician outcomes (e.g. clinician retention and clinical teaching time) higher than the guidelines 
writing group. The exercise provides some validation that the outcomes of interest to clinicians were also important to patients and families.

\section{Search Strategy for Systematic Review}

This review was in support of clinical practice guidelines for the SCCM which had no role in the review of evidence selected. In accordance with Preferred Reporting Items for Systematic Reviews and Meta- Analyses (PRISMA) guidelines, our systematic review protocol was registered with the International Prospective Register of Systematic Reviews (PROSPERO) on June 14, 2015 and was last updated December 8, 2015 (registration number CRD42015023445).

PubMed, CINAHL, and EMBASE databases were searched for quantitative studies in the area of family-centered care in critical care. We narrowed literature to English language studies with a publication date during or after 1994. The PubMed search strategy was:

((family-centered[Text Word] OR family-centred[Text Word] OR "Family/psychology"[Mesh:noexp] OR Professional-Family[TW] OR ("PatientCentered Care"[Mesh] AND ("Family"[Mesh] OR Family [TW] OR spouse[TW] OR significant-other [TW] OR parent[TW] OR child[TW] OR children [TW] OR sibling [TW] OR friend[TW]))) AND ("Intensive Care"[Mesh] OR "Intensive Care, Neonatal"[Mesh] OR "Intensive Care Units"[Mesh] OR "Critical Care Nursing"[Mesh] OR "Critical Care"[Mesh] OR critical care [TW] OR intensive Care [TW] OR Burn Unit[TW] OR Coronary Care[TW] OR Respiratory Care[TW] OR "ICU"[TW] OR "PICU"[TW] OR "NICU"[TW] NOT "Recovery Room"[Mesh]))

See Appendix $\mathrm{C}$ for search strategies in all databases. The searches were performed in December 2014. After searches in all databases, records were de-duplicated using "near match" in RefWorks ${ }^{\circledR}$. Investigators examined reference lists from previous systematic reviews for studies on family-centered care interventions $(12,14,15,17,18,21-24)$. Investigators searched the RefWorks ${ }^{\circledR}$ account for intervention terms using "All References" and the "Anywhere" field and sorted records into folders for each PICO question. Alerts were created and monitored by the librarian, and records uploaded when applicable until June 8, 2015. The Cochrane Central Register of Controlled Trials (CENTRAL) was searched for relevant registered trials; 154 records of trials were retrieved, of which 11 were in an ICU setting. An analysis of the predominant source titles was performed by the librarian using Web of Science for a date range of January 1994 through June 2015. We contacted known experts to inquire about unpublished studies that should be noted. We also searched Open Grey $<$ http://www.opengrey.eu/> and the New York Academy of Medicine's GreyLit.org for grey literature.

Eligible studies for this systematic review included randomized trials, and observational studies of family-centered care in an ICU setting that addressed the domains of interest. For interventions lacking experimental or observational studies, qualitative literature that helped to answer the PICO question was accepted but considered to be very low quality of evidence. We excluded studies where outcomes were not focused on the family or that were not conducted in an ICU environment (exception made a-priori: family presence at resuscitation in the emergency and pre-hospital setting). Proceedings that were not on topic or not original research or systematic reviews of original research were excluded.

Our December 2014 searches identified 4158 reports after de-duplication. Seventy-six additional studies were subsequently included from alerts and hand searches, and 236 studies were included in the final analyses. We used the GRADE tool to assess the level of evidence 
from included studies. Electronic Supplement Figure 2 describes the PRISMA Flow Diagram of study identification and inclusion.

\section{GRADE Process for Grading the Evidence}

GRADE assigns the strength of a recommendation based on the priority of a problem; balance of benefits and harms; certainty of the evidence of effect; values and preferences; equity; acceptability; and feasibility (25). The strength of the recommendation should summarize the evidence in a way that can be interpreted by individual clinicians dealing with specific problems under local conditions. Embedded in this summary is the understanding that the variables leading to the decision may vary under these same local conditions. For these reasons, a weak recommendation implies that clinicians consider the recommended action, but allows them leeway to make a decision based on the specific conditions they face. Strong recommendations imply greater applicability of the action across a spectrum of clinical situations.

Two or three investigators examined and summarized the literature relevant to each PICO question. To avoid intellectual conflict, guidelines writing group members who had authored a study of interest did not perform the GRADE analysis of their own scientific work. Standard GRADE methodology was used (see Appendix C). As with previous guidelines,(26, 27) a GRADE grid was used to help reach decisions on recommendations when consensus was not achieved or conflict arose (28). Each reviewing team was asked to consider whether publication bias might affect the findings. In addition, we searched for studies with federal funding for which there were no publications. Although publication bias can be difficult to assess, we did not find evidence for it.

The proposed recommendations were then put to a vote of the entire guidelines writing group. At least $50 \%$ of members had to vote in favor of, and no more than $20 \%$ against, each recommendation. In addition, a strong recommendation required at least $70 \%$ of those voting to vote in favor of the recommendation. Otherwise the recommendation qualified as weak. Strong recommendations use the language "we recommend," whereas weak recommendations use the language "we suggest" according to GRADE standards. All recommendations were based upon the published evidence and not from consensus statements. Voting results are posted in electronic supplement Table 3. A GRADE methodologist served as co-author on this project, attended all meetings, and provided oversight to the GRADE process to assure that recommendations were based upon appropriate evidence.

\section{Results: PICO Questions and Recommendations}

In the section to follow, the evidence summary and recommendations for each PICO question are presented. GRADE worksheets and voting summaries are archived with the SCCM. For all of the PICO questions, evidence varied significantly in study design and outcome measures, such that it was difficult to combine results statistically. For this reason, the nature of the individual studies is described in the narrative after each question.

\section{Executive Summary of Recommendations:}

All recommendations in these guidelines are made from moderate, low, or very low quality evidence and constitute weak recommendations per GRADE methodology (29). When outcomes are listed at the conclusion of a recommendation, it is because these outcomes have been tested (whereas others may not have been). We note the lack of research addressing the use of multiple simultaneous interventions. Although it seems likely that some combination of 
the interventions may improve outcomes, there are currently no data on the additive or synergistic effects of combined interventions. Clinicians and institutions will need to make a judgment about which intervention or combination of interventions are likely to be most successful in specific circumstances. We also note that adverse effects have not been described for most of the interventions and seem unlikely, but are possible. Statements as to adverse effects or risks for each PICO question are not repeated below, unless the intervention's specific adverse effects or risks were described. Recommendations apply to neonatal, pediatric and adult ICUs unless otherwise specified. When specified, it is because the evidence was available in only one age category.

\section{ICU Family-Centered Care Recommendations}

\section{We suggest the following:}

\section{Family Presence in the ICU}

1.1. Family members of critically ill patients be offered open flexible family presence at the bedside that meets their needs while providing support for staff and positive reinforcement to work in partnership with families to improve family satisfaction. (2D)

1.2. Family members of critically ill patients be offered the option of participating in interdisciplinary team rounds to improve satisfaction with communication and increase family engagement. (2C)

1.3. Family members of critically ill patients be offered the option of being present during resuscitation efforts, with a staff member assigned to support the family. (2C)

\section{Family Support}

2.1. Family members of critically ill neonates be offered the option to be taught how to assist with the care of their critically ill neonate to improve parental confidence and competence in their caregiving role and improve parental psychological health during and after the ICU stay. (2B)

2.2. Family education programs be included as part of clinical care as these programs have demonstrated beneficial effects for family members in the ICU by reducing anxiety, depression, post-traumatic stress, and generalized stress while improving family satisfaction with care. (2C)

2.3. Peer-to-peer support be implemented in neonatal ICUs to improve family satisfaction, reduce parental stress, and reduce depression. (2D)

2.4. ICUs provide family with leaflets that give information about the ICU setting to reduce family member anxiety and stress. (2B)

2.5. ICU diaries be implemented in ICUs to reduce family member anxiety, depression, and post-traumatic stress. (2C)

2.6. Validated decision support tools for family members be implemented in the ICU setting when relevant validated tools exist to optimize quality of communication, medical comprehension, and reduce family decisional conflict. (2D)

2.7. Among surrogates of ICU patients who are deemed by a clinician to have a poor prognosis, clinicians use a communication approach, such as the "VALUE" mnemonic (Value family statements, Acknowledge emotions, Listen, Understand the patient as a person, Elicit Questions), during family conferences to facilitate clinician-family communication. 2C) 


\section{Communication with Family Members}

3.1. Routine interdisciplinary family conferences be used in the ICU to improve family satisfaction with communication and trust in clinicians, reduce conflict between clinicians and family members. (2C)

3.2. Healthcare clinicians in the ICU should use structured approaches to communication, such as that included in the "VALUE" mnemonic, when engaging in communication with family members, specifically including active listening, expressions of empathy, and making supportive statements around non-abandonment and decision-making. In addition, we suggest that family members of critically ill patients who are dying be offered a written bereavement brochure to reduce family anxiety, depression and posttraumatic stress and improve family satisfaction with communication.(2C)

3.3. ICU clinicians receive family-centered communication training as one element of critical care training to improve clinician self-efficacy and family satisfaction. (2D)

\section{Use of Specific Consultations and ICU Team Members}

4.1. Proactive palliative care consultation be provided to decrease ICU and hospital length of stay among selected critically ill patients (e.g. advanced dementia, global cerebral ischemia after cardiac arrest, patients with prolonged ICU stay, and patients with subarachnoid hemorrhage requiring mechanical ventilation). (2C)

4.2. Ethics consultation be provided to decrease ICU and hospital length of stay among critically ill patients for whom there is a value-related conflict between clinicians and family. (2C)

4.3. A psychologist's intervention be provided to specifically incorporate a multimodal cognitive behavioral technique (CBT)-based approach to improve outcomes in mothers of pre-term babies admitted to the NICU; furthermore, targeted video and reading materials be provided in the context of psychological support to mothers of pre-term babies admitted to the ICU. (2D)

4.4. Social workers be included within an interdisciplinary team to participate in family meetings in order to improve family satisfaction. (2D)

4.5. Family navigators (care coordinator or communication facilitator) be assigned to families throughout the ICU stay to improve family satisfaction with physician communication, decrease psychological symptoms, and reduce costs of care and length of ICU and hospital stay. (2C)

4.6. Spiritual support from a spiritual advisor or chaplain be offered to families of ICU patients to meet their expressed desire for spiritual care and the accreditation standard requirements. (2D)

\section{Operational and Environmental Issues}

5.1. Protocols be implemented to ensure adequate and standardized use of sedation and analgesia during withdrawal of life support. (2C)

5.2. Nurses be involved in decision-making about goals of care and be trained to provide support for family members as part of an overall program to decrease ICU and hospital length of stay and to improve quality of communication in the ICU. (2D)

5.3. Hospitals implement policies to promote family-centered care in the ICU to improve family experience. (2C)

5.4. Given the evidence of harm related to noise, although in the absence of evidence for specific strategies, ICUs implement noise reduction and environmental hygiene practices and use private rooms to improve patient and family satisfaction. (2D) 
5.7. Family sleep be considered and families are provided a sleep surface to reduce the effects of sleep deprivation. (2D)

A table of the summary of recommendations for future research can be found on Appendix D. Note that this list is not exhaustive in nature.

\section{Family Presence with Patients in the ICU}

\section{PICO Question 1.1}

In the critical care environment, does open family presence at the bedside (also called open visiting) affect family satisfaction?

\section{Evidence Summary:}

The majority of literature examining the effect of open family presence at the bedside is observational or descriptive in nature. Many families value the opportunity to be at the bedside (30-34) and sometimes report the need to safeguard the patient or be vigilantly present (35-37). However, open family presence policies can be challenging to staff and may be perceived to increase workload $(15,38)$ and staff stress $(39)$. Family presence is necessary for family engagement at the bedside, which has been demonstrated, when coupled with an educational program, to improve outcomes (40-42).

There are no randomized trials addressing the effect of open or flexible family presence on family-centered outcomes. Data from existing observational studies addressed family satisfaction with variable ICU accessibility. One study examined open versus restricted family presence and demonstrated no differences in information or comfort for the families (43). Another study exposed families to open family presence with one group also receiving an information booklet (44). When comparing unrestricted vs. open family presence, there was a statistically significant increase in 9 out of 11 measures of knowledge with open family presence. The sample of families $(n=50)$ who received the informational booklet were more knowledgeable about specific facts. A third study conducted a pre and post survey after implementation of flexible family presence. When minimal restrictions were lifted from family presence (during report times) family satisfaction improved without changing staff satisfaction (45).

The quality of the evidence is very low for family presence with patients because there are no randomized trials and the observational studies are of poor quality. Studies are limited by small sample sizes, inadequate reporting of study results, and methodological issues. It is therefore difficult to understand how open family presence affect families. The literature would at least suggest that open family presence are more beneficial for families and may affect staff workload or perception of workload. In the future shifting away from the term visiting, to family presence and/or engagement would better reflect alignment with the family-centered paradigm.

\section{Recommendation:}

Given the value family members place on family presence, their dissatisfaction associated with restricted presence, and the benefit of engagement associated with presence, we suggest that family members of critically ill patients be offered open flexible family presence at the bedside that meets their needs while providing support for staff and positive reinforcement to work in partnership with families. (2D) 
Further research is needed to understand the best ways to implement open flexible visitation and fully understand the impact on family member outcomes and conflict in the ICU.

\section{PICO Question 1.2}

Does family presence during interdisciplinary team rounds improve family psychological symptoms, family trust in clinicians, family satisfaction with and preferences for care or communication, family or clinician conflict, degree of shared decision-making (as a direct result of family participation), and family knowledge?

\section{Evidence Summary:}

The majority of literature examining family presence on rounds is descriptive or observational in nature and focuses on the needs, perceptions and preferences of family members and healthcare providers. Studies have been conducted in the adult (46-48), pediatric $(49,50)$ or neonatal $(51,52)$ ICUs. In general, family members are as satisfied or slightly more satisfied when they participate in rounds and tend to be more in favor of family participation than support the traditional rounds format that excludes family members. Family members who participate in family-centered rounds report slightly or somewhat greater understanding and involvement in decision making and satisfaction with provider communication than those who do not. There was inconsistency in reports of greater anxiety or stress or privacy concerns related to participation in rounds, with some studies reporting an increase and others reporting no difference. No studies examined family or clinician conflict or alterations in the quality of bedside teaching. There is limited description to distinguish between presence and active participation by family members. All studies had significant limitations in the use of non-validated measures and single item questions of different dimensions of family experience.

Randomized trials and observational studies assessing validated family-centered outcomes related to this important topic are lacking. One small single-center randomized crossover trial in the PICU setting compared parent $(n=27)$ and resident $(n=21)$ perspectives after rounds were randomly assigned to occur at the bedside or in the conference room for two consecutive mornings. Parents reported increased satisfaction with bedside rounds: increased understanding of what the doctor said, increased confidentiality and intimacy respected, more questions were answered, enough time was spent with them, their child was more respected, their problem was taken seriously, and increased understanding of tests and treatment plans. Parents preferred bedside case presentation, and $81 \%$ wished that the next case presentation would take place at their child's bedside.

Data from several small observational studies (53-56) suggest that preferences (53), satisfaction(54),(55)(56), involvement in asking questions and in decision making(53), support with decision making(56) are improved. Anxiety could theoretically increase or decrease with family presence on rounds compared with rounds without family presence (56). One study (57) found that family perceptions change over time: compared to later in the ICU stay, on the day of admission families understood the plan less, felt less comfortable asking questions, did not want bad news during rounds were more likely to be concerned about privacy and wanted more information after rounds. However, since all studies reported generally high levels of satisfaction with traditional rounds, most of the described differences were small.

The evidence is of low quality because the single randomized trial is of low quality as are the limited number of observational studies. Studies are limited by small sample sizes, inadequate reporting of study results, and methodological issues (crossover, lack of validated measures). These limited data suggest no immediate harm and there are no data on long-term psychological outcomes. Family members generally express a preference to have the option to be present. In one study (56), residents had slightly less satisfaction with bedside rounds with parents participating compared with parents present but not participating. There is no evidence 
to determine the relative risks, feasibility, burden or cost of family presence and participation during rounds compared to traditional rounds without family presence or participation.

\section{Recommendation:}

We suggest that family members of critically ill patients be offered the option of participating in interdisciplinary team rounds to improve satisfaction with communication and increase family engagement. (2C)

Further research is needed to fully understand the best method of including family members in medical rounds and the impact of this on family outcomes. Further research is also needed to understand potential benefits and burdens and long-term effects on family outcomes

\section{PICO Question 1.3:}

Does family presence during resuscitation affect: family psychological symptoms, caregiver burden, family trust in clinicians, family satisfaction with care, family satisfaction with communication, family or clinician conflict?

\section{Evidence Summary:}

Both family members and clinicians have strong opinions about family presence during resuscitation efforts and several national organizations, including the Emergency Nurses Association (58), the American Heart Association (59), the American Association of CriticalCare Nurses (60), the American Academy of Pediatrics (61), the European Federation of Critical Care Nursing Associations,(62) the European Society of Pediatric and Neonatal Intensive Care (62), and the European Society of Cardiology Council on Cardiovascular Nursing $(62,63)$, have made formal statements in support of family presence during resuscitation. There is a large body of descriptive and qualitative literature that explores the attitudes of family members and clinicians about this practice. However, there are few randomized trials or observational studies that assess family-centered outcomes related to family presence during resuscitation.

Numerous studies examining family member attitudes about family presence during resuscitation suggest a supportive attitude for this practice, and many family members express a desire to be present during the resuscitation of a loved one (64-74). In one study of deaths in the emergency department, family members of decedents reported the belief that their presence would have helped the patient as well as the belief that their presence during the resuscitation would have attenuated their grief (64). An evaluation of resuscitation in a pediatric intensive care unit identified similar findings, with family members who were present for resuscitation reporting the belief that their presence brought comfort to their child and that their presence helped them adjust to the loss of their child (68). Themes from the qualitative literature include the idea of being there for the patient, seeing firsthand what was happening during the resuscitation, and protecting and supporting the patient $(75,76)$.

Support for family presence among clinicians is not consistently positive, and studies suggest few institutions have adopted policies regarding the presence of family members during resuscitation in the intensive care unit or emergency department $(62,77-79)$. Survey data suggest that nurses have a more positive outlook about family member presence during resuscitation compared to physicians $(66,73,80-84)$. In addition, providers with limited experience with family presence are less supportive of this practice than those who have participated in resuscitation efforts that included family members $(73,85,86)$. Concerns about family presence include the possibility of family interference with procedures, impaired staff 
performance, psychological trauma for family members, and litigation that may arise following a resuscitation attempt $(66,67,80,81,83,87-103)$. In a study assessing family-witnessed resuscitation in 162 UK emergency departments, 57 departments reported adverse events associated with family member presence (104). Adverse events included family member distress, interference with resuscitation, distracting the team, and making inappropriate demands on the team. Only one emergency department reported any medico-legal issues related to family presence during resuscitation, and none of the emergency departments that permitted family-witnessed resuscitation had plans to stop this practice based on adverse events (104). Other studies evaluating implementation of family presence programs have not reported interruptions in care related to family member presence $(80,105)$.

To date, two randomized trials have addressed family presence during resuscitation in the emergency department $(106,107)$, but none have addressed this issue in the hospitalbased ICU. Both of these studies have significant limitations. The first trial only included 25 patients undergoing resuscitation at a single center (106). The authors assessed 8 different family member psychological measures and did not identify any significant differences between witnesses of resuscitation and the control group. The other randomized trial evaluating family presence during resuscitation only provided descriptive data, and no quantitative comparisons were made between family members who witnessed resuscitation and those who did not (107).

Although randomized trials in the emergency department and intensive care unit are lacking, research done in the out-of-hospital setting has offered some insights into family presence. In a cluster-randomized trial, family presence was evaluated during resuscitation of cardiac arrests occurring at home. Emergency medical service units ("mobile ICUs") assigned to the intervention asked family members if they wished to be present at the resuscitation (108). Ninety days after resuscitation, enrolled relatives completed the Impact of Event Scale (IES) and the Hospital Anxiety and Depression Scale (HADS) by telephone. Frequency of PTSDrelated symptoms was significantly higher in the control group than in the intervention group and also higher among family members who did not witness CPR compared to those who did. Family-witnessed resuscitation did not affect resuscitation characteristics, patient survival, or the level of emotional stress in the medical team (108). A study following long-term psychological outcomes in these family members found that at 1 year after the event, psychological distress was higher for those family members not offered the option to witness resuscitation: PTSDrelated symptoms, major depressive episode, and complicated grief (109).

Data from existing observational studies $(110,111)$ do not clarify or answer remaining questions about family presence during resuscitation. One study enrolled 50 family members of patients undergoing trauma resuscitation in a single center. They found no significant differences in family member anxiety, satisfaction, or well-being when comparing family members who were present for the resuscitation to those who were not. Another study collected ratings of satisfaction from parents of children in a pediatric ICU and compared ratings of satisfaction between parents present during resuscitation compared to parents not present. Sixty-four parents completed the self-developed survey of parental satisfaction, and no significant differences in satisfaction were found when comparing parents who were present during resuscitation to those who were not.

The role of staff in supporting family members is one important aspect of family presence during resuscitation that has also been left largely unaddressed in randomized trials or observational studies. Many institutions with policies or programs regarding family presence have mandated that when possible a nurse, chaplain, or other trained staff member be available to support family members who choose to witness resuscitation $(80,105,112-114)$. The presence of support personnel was also included in the existing aforementioned randomized trials $(106,107,109)$; however, the relationship between family-centered outcomes and the presence of a dedicated support person has not been well-described. Since most studies 
included an individual designated to provide family support, this fact should be considered in implementation of this intervention.

The quality of evidence is low as existing randomized trials are of low quality or completed outside of the ICU setting. Studies are limited by small sample sizes, inadequate reporting of study results, and methodological issues (crossover, early termination due to lack of experimental concealment).

\section{Recommendation:}

We suggest family members of critically ill patients be offered the option of being present during resuscitation efforts, with a staff member assigned to support the family. (2C)

Future research is needed to better understand the influence of family presence for resuscitation on patient and family outcomes and the best approach for supporting family presence.

\section{Family Support}

\section{PICO Question 2.1:}

Among families of ICU patients, does teaching family members to participate in patient care affect: family satisfaction with care, family self-efficacy, or time to do not resuscitate (DNR) order?

\section{Evidence Summary:}

We identified two randomized trials that addressed teaching families to participate in care in the $\operatorname{NICU}(115,116)$. Both studies evaluated satisfaction with participation and being provided information. One study, including 55 mothers in both the control and treatment group demonstrated that participation and presence in the NICU increased maternal satisfaction (116). The other study, $(n=133)$, demonstrated increased satisfaction with information, written pain control information, pain control information satisfaction, a sense that nurses showed the parent how to look for signs of pain and perform comfort techniques, but did not reduce maternal stress (115). In addition, there is one randomized trial comparing standard care versus basic developmental care and another comparing basic developmental care versus care based on the Newborn Individualized Developmental Care and Assessment Program (NIDCAP) including behavioral observations (117). Both had sample sizes over 100. Parents of the infants born $<32$ weeks gestational age received questionnaires after the first week of admission in the neonatal unit and approximately 2 weeks after the birth of their infant. Results showed no significant difference in parental stress with these interventions.

Data from existing observational studies $(118,119)$ does not clarify or answer questions about family self-efficacy or time to DNR order. One study evaluated opinions of family members and desire to help with care in the ICU and $84 \%$ of families expressed a wish to be engaged in care (118). Another study involving 216 parents aimed to evaluate the impact of a national program designed to promote family-centered care in NICUs and to provide information and comfort to families during the NICU hospitalization of their newborn (119). Results of the study revealed better communication with the national program in place.

The majority of literature examining teaching family members in the ICU to participate in patient care is limited to two randomized trials and several observational studies focusing mainly on the neonatal setting. Maternal and other family participation in providing patient care in the $\mathrm{NICU}$ is strongly correlated with increased maternal satisfaction but does not decrease stress for the mother. 
The quality of evidence is moderate for satisfaction. Existing randomized trials only show benefit for this outcome. Observational studies are limited in number and in the provided evidence. Family members of NICU patients express the desire to assist with care and studies show increased satisfaction among mothers of those patients with this intervention.

\section{Recommendation:}

We suggest that family members of critically ill neonates be offered the option to be taught how to assist with the care of their critically ill neonate to improve parental confidence and competence in their caregiving role and improve parental psychological health during and after the ICU stay. (2B)

Further research concerning the benefits and limitations of family participation for care for children and for adult patients is needed.

\section{PICO Question 2.2}

Amongst family members of ICU patients, do training/education programs for family members affect family psychological symptoms; family stress; family satisfaction; family self-efficacy; and hospital length of stay and costs?

\section{Evidence Summary:}

The majority of literature examining the effect of family training/education programs is observational or descriptive in nature. There are three randomized trials addressing familycentered outcomes (40-42). The types of family training/education programs vary widely in type, duration, and purpose and as such are difficult to compare.

There are 7 studies addressing family psychological symptoms (primarily depression and anxiety) with family training/education programs. There are 3 randomized trials, one is of very low quality and found no effect of the training/educational intervention on family psychological outcome (40), while two randomized trials, one of high and one of moderate quality, found the COPE (Creating Opportunities for Parent Empowerment) educational program reduced family stress and depression $(41,42)$. There are also 3 observational studies $(120$, 121), (122), and a secondary analysis of a randomized trial (123) all of which found reduced anxiety. The observational studies are of very low quality.

Data from 4 studies address family stress with family training/education programs. There are two randomized trials $(40,41)$, a secondary analysis of a randomized trial (123), and one observational study $(124)$. The three studies $(40,41,123)$ used the COPE program. In secondary analysis of the randomized trial (123), maternal perceived stress was reduced by COPE training and maternal anxiety was directly associated with stress. In one randomized trial, the COPE training resulted in a decrease in several measures of stress (40); another study found the COPE training decreased stress at 2 months in but not in fathers (42). The observational study using an NICU education program found it reduced stress in fathers but not in mothers (124). The observational study and secondary analysis are very low quality, whereas the 2 randomized trials have high and moderate quality evidence. The results of the studies are conflicting with the 2 high quality studies showing reduced stress but one only in mothers and the other study having a small effect size.

Data from existing observational studies address family satisfaction with family training or education programs. There are four observational studies with very low quality evidence. One study assessed a family education program, finding the intervention group had a significant increase in family satisfaction with small effects (125). Another study used a family education program and found higher satisfaction in the experimental group with small effects (121). 
Data from one randomized trial and 3 observational studies address family self-efficacy with family training/education programs. The observational studies (126-128) are of low quality. One study used a program to teach parents how to do exercises with their pre-term children and found the parents performed the exercises on most days (93\%) and made few errors (126). A positive parent education program found the family was able to gain perspective on the situation and learn key concepts and find resources (127). A training program with families of children with tracheostomy found high satisfaction with the training (128).

Data from existing observational studies address cost and length of stay with family training or education programs. One observational study reported reduced median hospital length of stay by 24 days after the education program (129). A cost analysis of the COPE training found estimated cost savings of $\$ 4864$ per preterm born child with reduced hospital length of stay by 3.8 days (130). Both studies have very low quality of evidence.

The overall quality of evidence is low as there are predominately observational studies which are of low quality and 3 randomized trials, of which one trial is of low quality and two are of high quality. Studies are limited by small sample sizes, inadequate reporting of study results, and other methodological issues. The literature suggests that family education programs may reduce family psychological symptoms (depression and anxiety) and family stress. These may increase family satisfaction and family self-efficacy and may reduce hospital length of stay and costs.

\section{Recommendation:}

We suggest that family education programs be included as part of clinical care as these programs have demonstrated beneficial effects for family members in the ICU by reducing anxiety, depression, post-traumatic stress and generalized stress, while improving family satisfaction with care. (2C)

Further research is needed to understand the educational/training programs and their impact on family member outcomes and ICU length of stay and costs. In addition, much of the work to date has been done in pediatric populations; this research should be extended to adolescents and adults.

\section{PICO Question 2.3}

Amongst family members of ICU patients, does provision of family support such as family peerto-peer support, "date night", or family respite affect family psychological symptoms like PTSD, and family satisfaction with care?

\section{Evidence Summary}

We found no studies of single interventions of date-night or respite support. There are four studies of a single intervention of peer-to-peer support in the neonatal ICU. Three (131133) are descriptive, qualitative studies of small convenience samples in a single site that describe perceptions of health professionals $(132)$ or mothers $(131,133)$ receiving a peer-topeer counseling about breast feeding infants in the neonatal ICU. The mothers felt supported and satisfied to work with the peer counselors who had experienced themselves the difficulties of providing milk and breastfeeding in the neonatal ICU setting (131) and most ranked the peer support as most important for development of their maternal role in the neonatal ICU (133). The fourth descriptive study documented the utilization of peer-to-peer support provided in three formats: in groups, 1-to-1 at the bedside, and by telephone. Of the 477 parents (301 families) followed over a 2 -year period, $78 \%$ consistently used only one type of peer-support (bedside). A minority of families using the multiple types of support used them frequently. Of the 48 parents 
who completed the survey, $92 \%$ felt that the program met their needs and would recommend the program to another neonatal ICU family (134)

One small study (135) compared peer support for mothers of neonatal ICU infants at one site with a control group of mothers at another similar neonatal ICU site. The mothers at the site with peer support reported less stress at 4, less state anxiety, less depression, and more social support at 16 weeks than the mothers in the control group.

One small retrospective comparison study of peer support as part of a package of interventions to integrate parents into the care of neonatal ICU infants found that $92 \%$ would recommend the program to others (134). The evidence available for this topic is of very low quality.

These interventions, particularly peer-to-peer support, have low cost and low risk, with the suggestion of possible benefit. Qualitative research suggests that families value peer support in neonatal ICU (131-133).

\section{Recommendation:}

We suggest that peer-to-peer support be implemented in neonatal ICUs to improve family satisfaction, reduce parental stress, and reduce depression. (2D)

There is no evidence to evaluate date-night or respite and therefore no recommendation can be made about those topics at this time.

Further research is needed to evaluate the value of peer-to-peer support in all age groups to improve family-centered outcomes. Further research is also needed to evaluate family support options such as date-night or respite.

\section{PICO Question 2.4:}

Do written materials such as pamphlets, education materials, and bereavement materials targeting ICU family members improve psychological distress or communication compared to usual care?

\section{Evidence Summary:}

Ample evidence exists describing the many psychological, financial, and social challenges faced by the family members of ICU patients both during and after the period of ICU care (136-138). Two studies show that written materials can improve family member psychological distress. One randomized trial of 126 patients and their family members compared a bereavement brochure plus a proactive family conference to usual care finding that the intervention reduced PTSD, depression, and anxiety symptoms at 90 days after the patient's death (4). In another randomized trial including 175 family members, a family information leaflet compared to usual care improved family members' comprehension of medical information (139).

The quality of evidence is moderate because the results of these two studies demonstrated large treatment effects. Since studies were relatively small, no dose-response relationship could be tested. In addition, the strongest study (4) included two interventions - a proactive family conference and a bereavement pamphlet - making it impossible to determine the relative effectiveness of each intervention. The benefits seem quite strong and compelling with no associated risk or burden for relatives and can be implemented at relatively low cost.

\section{Recommendation:}

We suggest that ICUs provide family with leaflets that give information about the ICU setting to reduce family member anxiety and stress. (2B) 
Further research is needed to examine the relative benefits of different approaches to providing written materials to family members.

\section{PICO Question 2.5:}

Among family members of ICU patients does an ICU diary program improve/affect psychological symptoms (PTSD, anxiety, or depression)?

\section{Evidence Summary:}

Ample evidence exists describing that post-ICU burden for relatives, especially in the form of anxiety, depression and PTSD, in the months that follow the patient's discharge or death (136-138). During the patient's stay, families have difficulty in understanding and assimilating information, in maintaining a connection with the critically ill patient, and they may also question the quality of care $(140,141)$. All this contributes to the development of post-ICU burden after patient's discharge or death. The ICU diary is a diary that is written for the ICU patients during their time of critical illness. It can be written by relatives, nurses, physicians and others. Two randomized trials show that, in family members, receiving an ICU diary can reduce the risk of post-traumatic stress 3 months (142) and 12 months (143) after the patient's discharge or death. The quality of evidence is low with sample sizes of 36 and 143 respectively. These trials are conducted in single center or included only 2 sites. Across studies, the procedure involved in providing the patient or family with a diary is very different. In one trial (142), the diary was given to the patient by a research nurse or doctor who discussed its content with the patient. In the other trial (144), the diary was handed to the patient as the patient left the hospital with no discussion. Although both trials assess the impact of the diary on post-traumatic stress symptoms, they do not use the same outcome scales. The limits of both trials suggest the need for more research such as a multicenter randomized trial to further assess the effect of a diary on patients' relatives' wellbeing. Qualitative studies show that relatives describe the benefits of ICU diaries (144-146). No harm has been described and relatives mostly describe improved communicative, emotional, and humanizing experiences including improved comprehension of information, connection to the patient, and trust in caregivers. No risks or burdens for relatives have been described (see qualitative literature: (144-146)). There is a potential time investment to hospitals and ICUs for development of diary programs. The overall quality of evidence for ICU diaries is low.

\section{Recommendation:}

We suggest that ICU diaries be implemented in the ICU to reduce family member anxiety, depression, and post-traumatic stress. (2C)

Further research should be developed to assess the effectiveness and cost-effectiveness of a diary on family outcomes.

\section{PICO Question 2.6:}

In the ICU environment, do decision support tools for families or shared decision making itself improve/affect communication, cost or length of stay?

\section{Evidence Summary:}

Surrogate decision makers of ICU patients face significant challenges during hospitalization of their loved ones. Stress, emotion, the complexity of the clinical situation, uncertainty, poor communication quality, and lack of social support all present barriers to good quality decision-making. One published paper describes a small before-after study of a written shared decision support aid designed for surrogate decision makers of patients with chronic 
critical illness (147). The study took place in three academic medical centers. The decision support tool was associated with lower clinician-family discordance about prognosis, better quality of communication, better medical comprehension. Hospital costs were approximately $\$ 68,000$ lower in the intervention group. Quality of evidence is very low due to the small size of the study and the before-after design.

\section{Recommendation:}

We suggest that validated decision support tools for family members be implemented in the ICU setting when relevant validated tools exist to optimize quality of communication, medical comprehension, and reduce family decisional conflict. (2D)

Further research is needed to assess the effect of a decision aid on surrogate decision makers' wellbeing and the quality of decisions, as well as the important components of decision aids for improving outcomes.

\section{PICO Question 2.7:}

In the ICU environment, do clinician support tools targeting family support or primary palliative care such as checklists, worksheets and mnemonics improve family satisfaction, communication or psychological distress compared to usual care?

\section{Evidence Summary:}

Several studies have examined the impact of clinician support tools targeting families on patient-centered outcomes $(75,148,149)$. One observational study examined the impact of implementing a bundle of interventions (ICU Quality Bundle) on family satisfaction. The ICU Quality Bundle was a multi-faceted educational intervention targeting improving communication with family members during their loved one's admission to the ICU, and included a family conference at 72 hours, documentation of surrogate decision maker, and documentation of advanced directives, among other aspects. Eighty-six individuals in the intervention period were compared to 26 in the pre-intervention period. The intervention was associated with nonstatistically significant differences in family satisfaction within 72 hours from admission. Authors propose that non-significance was due in part to poor protocol compliance initiating conferences when indicated (148). Another multicenter randomized trial allocated families of ICU patients to a combined intervention of a bereavement packet and family conference, described above, focused on end-of-life care where clinicians employed the VALUE mnemonic. The intervention significantly reduced symptoms of PTSD, depression, and anxiety among family members at 90 days after the patients' death (4).

The evidence is of low quality. The observationtal study was rated very low due to study design, although it could be very low do the imprecision of effect (148). The single randomized trial provided the most compelling evidence of benefit of the mnemonic VALUE. In addition to use of VALUE during the family conference, the intervention also included a bereavement packet. It is impossible to determine if the effect is attributable to the use of the mnemonic or the bereavement packet or both. The study was upgraded because it identified several large, and clinically meaningful improvements in family outcomes. Benefits seem quite strong and persuasive without known risk or burden for relatives and likely can be administered at very low costs.

\section{Recommendation:}


We suggest that, among surrogates of ICU patients who are deemed by a clinician to have a poor prognosis, clinicians use a communication approach, such as the mnemonic "VALUE", during family conferences to facilitate clinician-family communication. (2C)

Further research is needed to identify the most effective methods to improve communication with family members of critically ill patients.

\section{Evaluation of Interventions Focused on Improving Communication}

PICO Question 3.1: In the ICU setting, do routine interdisciplinary family conferences improve patient or family outcomes, including increasing family satisfaction with communication and trust in clinicians, and reducing conflict between clinicians and family members?

\section{Evidence Summary:}

The effect of routine interdisciplinary family conferences on family satisfaction with care was addressed in one observational study (150). In comparing family members of 106 patients from Neurological and Medical ICUs who did not participate in any formal family meetings to those who did participate, those who did not participate were less likely to feel completely satisfied with the concern and caring shown to them as family members by ICU staff. Four different papers conducted analyses from an observational study of 51 ICU family conferences showing that family satisfaction with communication during family meetings was associated with improved communication techniques ( 2 with mixed qualitative-quantitative designs $(151,152)$ and 2 with quantitative, observational designs $(153,154))$. One study (153) found that an increased proportion of family speech during meetings was significantly associated with increased family satisfaction with physician communication. In another (151), there was a significant association between the number of empathic statements made during family conferences and higher degree of family satisfaction with communication. In the latter study, there was a dose-response gradient such that more empathic statements were associated with greater satisfaction. Furthermore, specific statements were associated with increased family satisfaction, including assurances of non-abandonment; assurances of comfort and not suffering; and support for family's decision to withdraw or not to withdraw life support (152). A third analysis (154) found that higher levels of shared decision making were associated with greater family satisfaction with communication. Findings from these studies provide indirect evidence that interdisciplinary family conferences, when conducted well, are associated with increased family satisfaction with communication.

Quality of communication was also associated with family conferences using medical interpreters in three different observational studies (155-157) during which interpreters were used for non-English-speaking families. Alterations in interpretation (i.e., additions, omissions, substitutions, and editorializations) were present in 55\% of communication exchanges between clinicians and family members (155); three-quarters were judged to have potentially significant consequences on conference goals, and 93\% were considered negative. In addition, nonEnglish-speaking family members received significantly less information and support than English-speaking family members (157). Another study (156) quantified examples of good quality of communication from qualitative content audiotaped in family conferences with Spanish- and English-speaking families. Overall quality of communication was found to be poor in both interpreted and non-interpreted ICU family meetings, but worse with Spanish-speaking patients.

Regarding conflict, in a before-after study (158), use of a proactive interdisciplinary (represented by the various disciplines involved in the patient's care) family conferences 
decreased the rate of family "non-consensus" from 1.7 to 0.09 days per patient. In an observational study, an increased proportion of family speech was also associated with decreased family ratings of conflict with clinicians (153).

ICU length of stay was addressed as an outcome associated with having family conferences in 4 studies, 3 of which were before-after studies (158-160) and 1 of which was a retrospective chart review (161). In the largest and only multi-center study, there was no significant difference in LOS between usual care (with or without a family meeting) and a system-level intervention that included formal family conferences (159). However, a singlecenter study of a similar intervention (158) found a significant decrease in LOS for those who received physician-led conferences during the "intensive communication period." In a 4-year follow-up of this same study (160), the difference in LOS between the intervention groups at each time period was not significant. In a retrospective chart review of PICU patients, (161) families who had a family conference were more likely to be discharged to a "new environment" (e.g., if the patient had been living at home and was discharged to a long-term care facility) versus families who didn't have a family conference though there were likely multiple confounders to this association beyond presence of a family conference. The overall quality of evidence for family conferences is low. There are no data to identify the most effective clinician participants or the most effective timing or frequency of family conferences.

\section{Recommendation:}

We suggest routine interdisciplinary family conferences be used in the ICU to improve family satisfaction with communication and trust in clinicians, reduce conflict between clinicians and family members. (2C)

Further research is needed to identify the most effective and cost-effective ways to implement routine family conferences in the ICU setting.

PICO Question 3.2: Amongst healthcare clinicians in the ICU, do specific communication techniques such as active listening, empathy and empathic statements, provision of supportive comments, language translation or cultural mediation affect family psychological symptoms, family satisfaction with care, communication or decision-making, physician-family conflict, or ICU utilization (length of stay)?

\section{Evidence Summary:}

Numerous observational studies have suggested the need for improved communication with family members $(137,162-167)$. One multicenter observational study analyzed recordings of 51 family conferences and published results in a series of articles (151-153). As stated in section 2.1, these studies showed that higher family satisfaction was associated with several features of high quality communication including higher proportion of family speech, increased empathic statements, and with specific statements of support regarding non-abandonment, symptom control, and support in decision-making. In addition, higher proportion of family speech was associated with fewer family reports of conflict with physicians.

A before-after study of an intervention to train multidisciplinary teams of ICU clinicians in a standardized approach to communicating serious news using the "SPIKES" approach (Set-up, Perception, Invitation, Knowledge, Emotions, Summarize)(168) showed higher family satisfaction with decision-making in the post-intervention period(169). The study did not show significant improvement in overall family satisfaction in the post-intervention period.

Review of the literature identified only one multicenter prospective randomized trial, which explored the impact of specific communication strategies on family-centered outcomes. This study (4) showed that providing relatives of patients who are dying in the ICU with a 
brochure on bereavement and using a proactive communication strategy for family conferences ("VALUE" --Value, Acknowledge, Listen, Understand and Elicit) resulted in longer conferences and more time for family members to talk. The authors found that family members in the intervention group reported fewer symptoms of PTSD, depression and anxiety 3 months after death in the ICU: they demonstrated a $22-27 \%$ absolute reduction in family reported symptoms of PTSD, depression and anxiety) (4). However, in this single study family members experienced very high levels of PTSD, depression, and anxiety in the control group.

The quality of evidence is collectively low. Only one randomized trial assessed the outcome of a specific communication strategy on family outcomes. Its quality of evidence was downgraded to "low quality" due to the high level of symptoms in the control group and the relatively small sample size limiting statistical stability.

\section{Recommendation:}

We suggest healthcare clinicians in the ICU use structured approaches to communication such as that included in the "VALUE" mnemonic when engaging in communication with family members, specifically including active listening, expressions of empathy, and making supportive statements around non-abandonment and decision-making. In addition, we suggest that family members of critically ill patients undergoing withdrawal of life support are offered a written bereavement brochure to reduce family anxiety, depression and post-traumatic stress and improve family satisfaction with communication. (2C)

Further research is needed to identify the most effective ways to improve communication with family members and which components of a communication strategy are most effective.

\section{PICO QUESTION 3.3:}

In the ICU environment, do communication-training programs for clinicians, such as education or simulation improve family psychological symptoms, family-rating of quality of dying, patient or family rated quality of communication, family satisfaction with communication, clinician selfefficacy, or clinician psychological symptoms?

\section{EVIDENCE SUMMARY:}

Ample evidence exists describing the importance that patients and families place on high-quality communication in the $\operatorname{ICU}(26,27,170,171)$. Furthermore, additional evidence demonstrates that poor communication can cause patient and family dissatisfaction (162-165) and even harm in the form of anxiety, depression, symptoms of PTSD and complicated grief $(137,166,167,172)$. Given the importance of high-quality staff-family communication to familycentered outcomes, many investigators have examined communication training programs as an intervention to improve the quality of communication in the ICU setting. The training programs examined in the literature vary widely, with training interventions ranging from purely didactic to simulation-based. Additionally, the training programs intensity differed from a 1-hour workshop to a 12-month didactic series. All studies that examine the effect of training programs on clinician self-efficacy showed a significant improvement in clinician-reported communication skills and comfort with family communication training (169, 173-182). Despite this consistent self-reporting of improved skills and comfort with communication, only one of two studies examining the effect of communication training on staff anxiety demonstrated improvement in staff-reported anxiety with family communication (180), while the other study found no 
improvement (183). Importantly, while clinicians consistently self-report improvement in communication skills and comfort with communication, studies that employed more objective third party measurements of the quality of communication showed mixed results, with at best only modest improvements in a limited number of communication elements. Of note, the studies that demonstrated at least some improvement were of a longer training duration (1 to 12months) $(173,178,182,183)$, in contrast with a 1-hour (176) and 1-day (184) training that showed no improvement. Additionally, one study found that the improvement in quality of communication seen immediately after the training was lost at a 6-month follow-up (184).

Only two studies examined family-reported outcomes. One of these studies found potential improvements in some aspects of family satisfaction with communication (frequency of nursing communication, frequency of physician communication, and honesty of communication after a training program intervention. Due to the very low quality of the evidence, it is difficult to draw conclusions based on this single study (169). The other study showed no significant improvement in patient) or family satisfaction with communication or with patient- or family-rated quality of end-of-life care with high quality data (185). In fact, surprisingly, this same study showed an increase in patient-reported depressive symptoms for those patients assigned to the clinician group who had received the communication training intervention. Therefore, educational interventions have been shown to improve clinician-reported self-efficacy in communication skills and comfort with family communication as well as objectively documented communication skills, but these interventions have not been shown to improve patient- or familyreported outcomes.

The quality of evidence is very low for this PICO question for several reasons. First, although "clinician self-efficacy" was consistently shown to improve with the intervention (often with a large magnitude of effect), the quality of evidence from these studies was generally limited due to pre-post designs with lack of non-intervention controls, relatively small study numbers, and use of non-validated surveys. Second, studies examining other outcomes of interest showed inconsistent family reported results with no clear direction of evidence.

Intuitively, patients and families would benefit from clinicians who are well-trained in family-centered communication. It has been established that the risks and burdens of poor communication include patient and family dissatisfaction, anxiety, depression, PTSD and complicated grief $(137,162-167,172)$. Communication training for ICU clinicians is seemingly a low risk intervention. Costs are unknown and may range from minimal to considerable depending on the nature of the training. At this time, best practices for specific communication training programs are not well established in the literature.

\section{Recommendation:}

Based on existing evidence of patient and family burdens associated with poor communication, as well as improved clinician-reported skills and comfort following communication training, we suggest that ICU clinicians receive family-centered communication training as one element of critical care training. However, no recommendation can be made to suggest the use of any of the specific communication training programs that have been evaluated based on existing evidence. (2D)

Further research is needed to determine if and how communication-training programs can be implemented to improve patient- and family-centered outcomes. Important components of future research include validation of a quality of communication measurement tool for consistency of reported outcomes, increase in the use of family-reported outcomes as the standard for quality 
of family communication; inclusion of data on cost of implementing communication training programs; more long-term follow up studies since skills may dissipate over time; comparison of different communication teaching modalities such as didactic training, role-plays and simulation, and further investigation of possible risks to patients such as increased depression (185).

\section{Evaluations of the Use of Specific Consultations and Team Members}

\section{PICO Question 4.1:}

Among family members of ICU patients does a palliative care consultation impact ICU and hospital utilization?

\section{Evidence Summary:}

There are limited data including five very low to low quality studies that suggest that proactive consultation of palliative care decreases ICU and hospital length-of-stay among certain patients, such as patients with advanced dementia, global cerebral ischemia after cardiac arrest, patients with prolonged ICU stay, and patients with subarachnoid hemorrhage $(\mathrm{SAH})$ requiring mechanical ventilation (186-190). However, one study showed no significant changes in ICU and hospital length of stay (189) and another showed a significant decrease in ICU length-of-stay but no change in hospital length-of-stay (188). Quality of the evidence is low as all studies used a non-randomized, before-after study design, sample sizes were relatively small (under 100 patients in each study arm), and there were some inconsistent results. Although data from different studies varied, studies showed a decrease in ICU and in hospital length of stay. The risks involved in this intervention, and the other interventions in these guidelines, are mostly in terms of costs and specifically the opportunity costs for the resources involved in implementation of the intervention. Increasing palliative care consults may incur a cost saving in ICU and hospital length of stay (191).

\section{Recommendation:}

We suggest proactive palliative care consultation be provided to decrease ICU and hospital length of stay among selected critically ill patients (e.g. advanced dementia, global cerebral ischemia after cardiac arrest, patients with prolonged ICU stay, and patients with subarachnoid hemorrhage requiring mechanical ventilation). (2C)

Further research is needed to better understand the effective and cost-effective components of a palliative care consultation and to identify the patients for whom palliative care consultation is most beneficial.

\section{PICO Question 4.2:}

Among family members of ICU patients does ethics consultation impact family satisfaction or ICU or hospital length of stay?

\section{Evidence Summary:}

There are limited data reporting the effect of ethics consultation on family satisfaction, with only one single-center randomized trial that assessed the impact of ethics consultation on 
family satisfaction showing no significant change (192). There are two trials in which family satisfaction was examined in the intervention arm but not the control arm; family satisfaction appeared high in both these studies $(193,194)$.

There are 4 studies of diverse methodological quality, each testing slightly different types of ethics consultation in different populations for effect on length of stay (192-195). Three of the four studies reported shorter ICU and/or hospital length of stay among patients receiving ethics consultations. The effect appeared strongest in studies in which the trial involved ethics consultation in response to clinician-family conflict, rather than using ethics consultation preventatively to improve communication. An important limitation is the heterogeneity in the nature of the deployed intervention. The highest quality study, which randomized 551 patients, showed only a relatively small effect of ethics consultation on ICU length of stay (194). The studies that showed the greatest effect had the smallest sample size and had the greatest methodological weaknesses. There are financial and opportunity costs of increasing the size and scale of ethics consultation services in hospitals to meet the demand of the large group of ICU patients that would be eligible and no evidence of harm. Increasing ethics consults may incur a cost saving in ICU and hospital length of stay. The overall quality of evidence for ethics consultation is low.

\section{Recommendation:}

We suggest that ethics consultation be provided to decrease ICU and hospital length of stay among critically ill patients for whom there is a value-related conflict between clinicians and family. (2C)

Further research is needed to better understand the effective and cost-effective components of ethics consultations and to identify the patients for whom ethics consultation is most beneficial.

\section{PICO Question 4.3:}

Among family members of ICU patients does a psychologist consult improve/affect family outcomes?

\section{Evidence Summary:}

Access to psychologists for ICU family members appears limited; psychology consultation service provision range from 4\% in the United Kingdom (196) and United States (197) to $29 \%$ in Australia (198) for pediatric ICU patients. In one study of adult ICUs in Europe, $37 \%$ of relatives had access to psychologists (172). Only three publications describing two interventions were considered evidence to assess the effect of psychological consultation. All three studies assess the effect on mothers' symptoms in the context of pre-term neonates admitted to neonatal ICUs. One randomized trial compared 36 mothers supported by a psychologist during the infant's hospitalization to 23 mothers receiving psychologist support and reading and video material dealing with specific subjects related to prematurity, affective mother-child connection, and care of a premature baby. The group receiving additional materials in this study had more favorable anxiety levels at follow up (State-Trait Anxiety Inventory) (199).

Another randomized trial compared 43 mothers undergoing 6 support sessions to 62 mothers whose 6 sessions included targeted specific psychological interventions, namely trauma-focused cognitive behavioral therapy (CBT). Targeted therapeutic sessions included: psychoeducation about PTSD, cognitive restructuring, narrative and muscle relaxation. The first publication (200) describes outcomes at 4-5 weeks after the birth of the pre-term infant compared to the time of the intervention, and the second (201) describes the maintenance of 
improved outcomes at 6 months. The targeted session improved all spheres of evaluated outcomes including Davidson Trauma Scale, Beck Anxiety, and Beck Depression Inventory.

One small observational study has been conducted in trauma patients in the adult ICU. Early psychological support was associated with a nonsignificant reduction in, and depression, significantly reduced PTSD and increased use of psychiatric medications at one year (202).

Quality of the evidence is very low for the study of supplementary informational material; it is a single study (little evidence), the timing of the intervention is not described and effect of intervention is not reported (study design limitation). The study of trauma-focused CBT (201) is low quality evidence (observational study, single center, small numbers). The greatest improvement was found in the mothers that were the most distressed (and thus the most likely to not be functional in their parental role).

Uncertain benefit, low cost and low risk were noted in the study describing the provision of additional informational materials.

\section{Recommendation:}

We suggest a psychologist's intervention be provided to specifically incorporate a multimodal cognitive behavioral technique (CBT)-based approach to improve outcomes in mothers of preterm babies admitted to the NICU. Furthermore, we suggest that targeted video and reading materials be provided in the context of psychological support to mothers of pre-term babies admitted to the ICU. (2D)

Further research testing the impact of psychological support in adult and pediatric populations, and larger studies in the pre-term neonatal population are needed.

\section{PICO Question 4.4:}

Among family members of ICU patients does a social work consultation impact family satisfaction?

\section{Evidence Summary:}

One large randomized trial involving 873 (203) assessed the value of a social worker to provide support for family members; one small observational trial addressed the effect social workers have on family satisfaction (204). The trial showed a non-significant improvement in satisfaction with, satisfaction with the amount of information provided, or satisfaction with involvement in decision making. In the observational study the presence of social workers independently predicted increased family satisfaction. Quality of the evidence is very low as there is only the single randomized trial and a small, very low quality observational study. The cost of utilizing social workers in this way is not known and would depend in part upon whether they are already available in the ICU.

\section{Recommendation:}

We suggest social workers be included within an interdisciplinary team to participate in family meetings in order to improve family satisfaction. (2D)

Further research is necessary to evaluate outcomes associated with social worker interventions.

\section{PICO Question 4.5:}

Among family members of ICU patients does a navigator (care coordinator or communication facilitator) improve family anxiety, depression, post-traumatic stress, family satisfaction, family or clinician conflict or resource utilization? 


\section{Evidence Summary:}

One moderate quality study suggests a navigator may decrease psychological symptoms in family members (205). This randomized trial assessed the effect of communication facilitators, trained to identify and support family members' communication styles and to identify and mediate conflict, who met with family members regularly in the ICU (and 24 hours post discharge) to support communication between the family and the ICU team. This intervention was associated with a reduction in depression severity, among family members at 6 months, albeit with limited follow-up(205). There was no significant reduction in anxiety or PTSD at 3 or 6 months (205). Another study assessed limited (1-2 visit) nurse liaison involvement aimed specifically family anxiety related to ICU-ward transfer; the intervention did not alter state-trait anxiety inventory scores at 3 months (206).

Two publications derived from the same study address physician and nursing communication and satisfaction with that communication by families $(207,208)$. Families and staff were surveyed before and after integrating a family support coordinator (a nurse with ICU experience and psychological/social work training) assigned to families of patients who were expected to require ICU care for 5 days or were at high risk of death; the coordinator's role included identifying information needs, clarifying medical information, assisting in decisionmaking, organizing meetings with relevant team members, and relaying family needs to the team. Family members rated physician communication, social work communication, and respiratory care communication but not nursing communication more favorably after the involvement of a family support coordinator. Perception of family/physician conflict did not change from 'before' to 'after' the intervention of a family support coordinator.

The single randomized trial of communication facilitators in the ICU found a reduction in ICU costs among all patients and decedents. The intervention reduced ICU and hospital length of stay among. (205). In another before and after study, changes of ICU length of stay and ICU costs after the introduction of a family support coordinator did not achieve statistical significance. The analysis was not stratified by patient mortality (207).

The quality of the evidence is low for navigator impact on family psychological symptoms; the single randomized trial appears to improve family outcomes in terms of depression at 6 months, but had substantial loss to follow-up. The two studies addressing satisfaction were before/after design, and suffer from the imprecision of small numbers. The quality of evidence for evaluating resource utilization is moderate, as the data from a single randomized trial showed substantial reductions in ICU and hospital length of stay (among decedents only), and costs of care. The second study showed a similar trend but with no statistical difference in costs. Observational data suggest that timely (i.e. earlier) discussion of prognosis is essential to allow family members to prepare emotionally and logistically for the possibility of a patient's death (5). Potential benefits include satisfaction with physician communication, decreased family depression, and decreased length of stay, at potentially moderate personnel cost if hiring a family support coordinator is required.

\section{Recommendation:}

We suggest that family navigators (care coordinator or communication facilitator) be assigned to families throughout the ICU stay to improve family satisfaction with physician communication, decrease psychological symptoms, and reduce costs of care and length of ICU and hospital stay. (2C)

Further research is needed to examine the most effective and cost effective approach to training and implementing navigators, identify patients and family members most likely to benefit, and describe any potential adverse outcomes associated with navigators interacting with families. 


\section{PICO Question 4.6:}

Among families of ICU patients, does routine consultation of a spiritual care provider improve outcomes?

\section{Evidence Summary:}

Many families express that they value spiritual care $(26,209,210)$ and the availability of a spiritual advisor or clergy (211-213). Spiritual care has been identified as a core element of palliative care (18). The Joint Commission requires that patients receive a spiritual assessment (PC.01.02.01.04), that hospitals provide care that accommodates spiritual end-of-life needs (PC.02.02.13.01) and that patients have a right to spiritual and religious services (RI.01.01.09) (214).

Many families interact with spiritual care providers during a patient's ICU stay (215). In one study, patients on telemetry units were visited by chaplains, and perceived their interventions as helpful (216). No evidence exists comparing the intervention of a spiritual care provider versus standard care for patient and family outcomes. One observational study describes the types of activities spiritual care providers engage in, and found that, for families that interacted with spiritual care providers, increased overall family satisfaction with ICU care was associated with discussions about the patient's wishes for end-of-life care and a greater number of spiritual care activities performed (215). In another observational study in which family members $(n=356)$ were surveyed about spiritual care, family members had greater satisfaction with spiritual care if a spiritual advisor or pastor was involved within 24 hours of the patient's death (217). Provision of spiritual care and satisfaction with the ICU experience were strongly correlated (217). A before-after study described the involvement of a comprehensive support care team, which included a chaplain, and resulted in a decrease in intensity of care, as assessed by the average Therapeutic Intervention Scoring System (TISS) for patients (218).

Collectively the evidence is very low quality as no studies provided a control group and interventions did not consist solely of involvement of a spiritual care provider. There is probable benefit with low risk of harm. Additional costs may be incurred in settings where spiritual care providers are not already available.

\section{Recommendation:}

Given the consistency of expression of family values for availability of spiritual care, the accreditation standard requirements, and the association with increased satisfaction, we suggest that families be offered spiritual support from a spiritual advisor or chaplain. (2D)

Further research testing the impact of a spiritual care provider on patient and family outcomes is needed. The best method for provision of spiritual support has not been studied and warrants further investigation.

\section{Operational and Environmental Issues}

PICO Question 5.1:

In the ICU environment, do protocols for withdrawing life support improve outcomes?

\section{Evidence Summary:}

Only four articles were found that specifically addressed the use of protocols for withdrawal of life support $(149,219-221)$ Of these, three directly addressed withdrawal of life 
support $(149,219,220)$ while one (221) addressed the impact of a protocol on sedation and analgesia use. The first three studies addressed only the documentation of protocol use. No measures of family satisfaction were directly obtained, although one study showed high levels of clinician satisfaction(221) This study evaluated the use of a protocol for sedation and analgesia in a before-after study design showing increased use of sedation and analgesia without hastening. High levels of clinician satisfaction were reported with the protocol, supporting the use of a protocol for managing patient symptoms.

The evidence on protocols for withdrawal of life support is of low quality. Only one study, a before-after design (221), examined outcomes demonstrating that protocols led to increased use of sedation and analgesia without affecting time to death or ICU LOS. Quality of dying and death was not significantly improved. The benefits of a protocol potentially optimizing sedation and analgesia are persuasive. Although family outcomes have not been explicitly examined in existing studies, family burden of poor withdrawal of life support caused us to include this recommendation.

\section{Recommendation:}

We suggest that protocols be implemented to ensure adequate and standardized use of sedation and analgesia during withdrawal of life support. (2C)

Further research is needed to assess the effect of protocols for withdrawing life support on patient- and family-centered outcomes.

\section{PICO Question 5.2:}

Does the inclusion of nurses in ICU communication about decision making about the goals of care improves family-centered outcomes?

\section{Evidence Summary:}

While nurses are ubiquitous and integrally involved in all aspects of ICU care, this question searches whether or not specifically including nurses in communication about decisionmaking about the goals of care improves communication will improve family outcomes. In the ICU, inclusion of nurses in decision making may improve family-centered outcomes such as quality of communication, quality of care, family satisfaction with care, and conflict. The majority of literature examining the inclusion of nurses in ICU decision-making is descriptive or qualitative in nature (174, 222-225). These studies were conducted in adult, pediatric, and neonatal ICUs and focused on the quality of communication, quality of care, and family satisfaction with care.

In general, studies of initiatives to include nurses in decision making situations were preand post-intervention survey designs or descriptions of existing practice. There are no randomized trials on this topic. No standard intervention was used and therefore no specific training or involvement of nurses can be recommended. Nevertheless, the need for efforts to improve quality of communication is evident.

Two studies provide informative evaluations of interventions to increase nursing involvement in decision-making about the goals of ICU care. One single-unit study compared patients exposed to a multi-professional care communication team including clinical nurse specialists (along with unit-wide palliative care training) with patients who received standard practice. A statistically significant decrease in ICU length of stay and hospital length of stay and total costs of care (225) were described with the intervention. A small, single-unit study of 15 patients used a single nurse trained as a family support specialist finding that families and physicians reported improvements in the quality of communication and patient-centeredness (226). 
Quality of evidence is very low due to the limitations of the two relevant studies. While both report empiric evidence, the studies used before-after rather than randomized designs. Both had a relatively small sample of clinicians (e.g. physicians, nurses, therapists) and patients and did not attempt to adjust for confounding or other influences. The first study used unit-wide palliative care education and therefore the impact of each intervention alone (nursing involvement versus education) is unknown (225). The second (226) was conducted in a single unit, included only 15 patients, and relied on a single nurse interventionist. Therefore, it is unknown whether this result would be generalizable. No studies currently exist that examine the impact of such an intervention on family-reported outcomes other than family satisfaction with communication. One study suggested decreased ICU and hospital length of stay. There are limited data to estimate the relative risks, feasibility, burden or costs.

\section{Recommendation:}

We suggest that nurses be involved in decision-making about goals of care and trained to provide support for family members of critically ill patients as part of an overall program to decrease ICU and hospital length of stay and to improve quality of communication in the ICU. (2D) No recommendation can be made regarding decision-making due to lack of supporting evidence.

Further research is needed to examine the potential benefits of nursing involvement in ICU decision-making for family as well as clinician outcomes.

\section{PICO Question 5.3:}

Does a comprehensive "family-centered care" approach to ICU care improve family-centered outcomes during critical illness?

\section{Evidence Summary:}

Some ICUs have instituted procedures and policies meant to improve the unit's entire approach to family-centered critical care. These policies are intended to shift the paradigm of critical care away from paternalistic or hierarchical care and toward an approach that integrates families as care partners in the ICU (227). Limited empiric evidence exists evaluating the effect of the comprehensive programs on outcomes important to families. One study developed and implemented a Family Support System in a single neonatal ICU that consisted of three components: (1) new policies regarding flexible family presence at the beside, unit communication, and team rounding promoting family involvement, (2) identification and referral of parents at high risk of psychosocial distress to social workers, and (3) a post-ICU stay home care visitation program (228). The authors sought to measure the effects on parental hypervigilance and anxiety, using hospital resource utilization as the primary outcome. There were 80 patients included in the control (pre-intervention) arm and 90 patients who received the intervention. Following introduction of the programmatic and policy changes, there was a reduction in hospital readmission days. A second study created and implemented a hospitalwide program intended to emphasize the importance of family members and to change policies to allow families to participate more fully in care (229). This included education of family members on their role, 24-hour family presence, and encouraging families to participate in bedside care. Family members could elect to participate in the program. The authors measured satisfaction with the program, with over $84 \%$ of participants reporting that it made the hospital stay more or much more positive. There was no reported comparison of overall satisfaction with care among those family members who did or did not participate. 
The quality of evidence is very low due to study design limitations and the data is limited to quality improvement project assessments. Both studies have limited generalizability as they were conducted within a single center and among a limited population of patients. One study used a pre- and post-implementation design (228) and the other did not compare the participating family members with a control group (229).

There is potential for burden on staff and family members, and the intervention is potentially costly depending on extent of program components, but there are unlikely to be important risks other than the opportunity costs.

\section{Recommendation:}

We suggest hospitals implement policies to promote family-centered care in the ICU to improve family experience. (2C)

Further research is needed to examine the effect of hospital and ICU policies designed to promote family-centered care. Additionally, future research should identify specific program components that drive improvements in family-centered outcomes.

\section{PICO Question 5.4:}

In the ICU do noise reduction strategies, private rooms or space for family members (beyond single rooms for patients) affect patient/family satisfaction, staff stress or noise?

\section{Evidence Summary:}

Ample evidence exists that noise levels are high in the ICU and negatively affect patient outcomes $(32,230-235)$. However, a paucity of data exists evaluating the effect of noise reduction on family-centered outcomes. Of the literature reviewed, only one study addressed family satisfaction with care associated with noise reduction (236). This randomized trial of cobedding multiple-gestation infants in the neonatal ICU versus bedding single infants alone (infants $n=37$, parents $n=19$ ) resulted in a statistically significant increase in parent affirmations of the questionnaire item "attempts were made to create a quiet environment for my baby" that was one component of a Family Satisfaction Score.

Single family rooms are associated with noise reduction, as well as greater family and patient privacy. In a study comparing family attitudes towards single family rooms compared to an open bay ward neonatal ICU design, families had increased satisfaction in several areas. These were in response to the questions "parent comfortable visiting", "privacy for bonding", and "could control light". Noise levels were 20 decibels higher in the open ward ICU (237).

The majority of literature examining space for family members is descriptive or qualitative in nature and limited to single-center studies. The focus of this work is the needs and impact on the family members and clinical staff satisfaction. Two descriptive survey studies included evaluation of the psychological symptoms of families using previously validated tools (sleep disturbance, Beck anxiety index and fatigue scales). While these described fatigue, lack of sleep, and anxiety among family members, they did not measure the impact of improvements in ICU design or space for family $(238,239)$.

Five additional studies attempt to provide information on ICU design using pre- and postdesign. One study evaluated three physical design layouts of a neonatal ICU using focus groups (240) of parents with surviving infants $(n=5)$ and staff $(n=11)$ of the unit. Designs studied included open space (multiple infants in a single, large room without parent beds), modified room (a curtained space for each infant that included a parent bed) and smaller room designs (a private room with a parent bed for each infant). The focus groups suggested that the smaller 
room design promoted bonding between parent and infant, led to better comprehension of illness among parents, and allowed healthcare providers to meet healthcare needs.

These findings were supported by other studies comparing an open bay and single family room in a neonatal ICU. A pre-post study measured baseline, 6 and 12-month family member and staff reaction to change to a single family room from open bay. Families of long-term stay infants in single family rooms were more likely to report privacy for bonding with the infant and control of lighting; surrogate markers for satisfaction. However, over 30 other surrogate outcomes were evaluated with non-significant results. There was also inconsistency in staff results at one year. For instance, there was a significant improvement in staff willingness to help each other but the work was reported as more physically demanding. Another study used similar methods, surveying staff members $(n=67)$ and family members $(n=85)$ longitudinally prior to and following a move from an open bay neonatal ICU to one with private rooms. Following the move to the single family room, family satisfaction related to design significantly improved, including perception of privacy at 6 months and 1 year and the opportunity to interact with other families (241). Of the clinician outcomes of interest to these guidelines, there was no long-term effect on staff stress.

Another pre-post relocation design study interviewed 103 hospitalized patients and families prior to and after a move to assess satisfaction with the new environment, namely privacy, space, noise, light and atmosphere. Additionally, the investigators used Hospital Consumer Assessment of Healthcare Providers and Systems (HCAHPS) and Press Ganey survey questions specific to environment to explore similar issues. The interviews revealed that the privacy of room, room size, and bathroom were key features that led to improved patient satisfaction. Areas of dissatisfaction included noise, lighting systems, television controls and chairs. The survey data revealed that there were improvements in satisfaction with every aspect of the environment of care following the relocation (231).

Study design and scope substantially limit the application of findings to other settings. There are no randomized trials of the effect of design changes or space for family members on family-centered outcomes. All studies are limited to a single center with small sample sizes and a narrow patient population. Most of this work has been described as quality improvement assessment rather than research.

Given the benefits of ICU noise reduction for patient clinical outcomes (e.g. delirium), noise should be addressed in all ICUs. There is a significant cost associated with building private rooms. However, building new ICUs private rooms are advocated by ACCM/SCCM for a variety of reasons (including infection control), only one of which is family-centered care outcomes (242), so the cost may be justified given the multiple benefits. The data available suggest harm in the form of staff stress that needs to be managed with a change from open bay neonatal ICUs to private rooms. There is no evidence to determine the relative risks, feasibility, burden or justification of cost of additional bedside space for family members. The overall quality of evidence for noise reduction strategies is very low.

\section{Recommendation:}

Given evidence of harm related to noise, although in the absence of evidence for specific strategies, we suggest ICUs implement noise reduction and environmental hygiene practices and use private rooms to improve patient and family satisfaction. (2D) No recommendation can be made for family space. However, it is noted that the SCCM/ACCM Guidelines for ICU design recommend designing new ICUs with family space based upon consensus statement (242). 
Further research is needed evaluating the effect on family-centered outcomes of interventions to reduce noise or improve the ICU environment in the ICU. Further research is also needed regarding the impact on outcomes of private rooms and family space and the effect of private rooms on family-centered outcomes.

PICO Question 5.5:

Do executive (hospital leadership) walk rounds improve family-centered outcomes in the ICU?

\section{Evidence Summary:}

Increased presence of hospital leadership in the form of executive walk rounds in the physical space of the ICU has been considered as a method of improving outcomes important to family members (243-246). Two primary investigations specifically measure the effect of such executive walk rounds on clinician burnout. Both of these articles demonstrated no clear evidence that executive walk rounds increases or decreases clinician burnout in small samples with design limitations preventing clear conclusions $(245,246)$.

Quality of evidence is very low due to limitations in the design of the two studies. One study used a pre/post design that included a small sample of clinicians and did not attempt to adjust for confounding. The executive walk rounds were merely one component of what the authors describe as a "comprehensive unit-based safety plan." Similarly, the second study examines surveys of clinicians in a convenience sample of 44 neonatal ICUs. Participants reported their exposure to executive walk rounds. Executive walk rounds were not prospectively tested nor could the authors account fully for other cultural or policy variations among ICUs. No studies currently exist that examine the impact of such an intervention on family-reported outcomes.

These limits suggest that there is a need for more research that tests executive walk rounds in a randomized trial, ideally on a multi-center scale and testing family-reported outcomes. Treatment effects are not reported in the literature. There is a potential for high burden to hospital leadership.

No recommendation can be made due to lack of supporting evidence.

Further research testing executive walk rounds on family-reported outcomes is needed.

\section{PICO Question 5.6:}

Does consistency in staffing improve family-centered outcomes during critical illness?

\section{Evidence Summary:}

Continuity of care in the ICU is defined as the establishment of a continuous caring relationship between the medical care team and the patient and family members, typically measured by the number of professionals who primarily care for the patient over a given period of time $(247,248)$. Two studies tested the effect of aspects of this continuity of care on family satisfaction with communication or ICU length of stay $(247,249,250)$. One study introduced a care model emphasizing consistency of care for long-term pediatric patients in a single ICU in a pre- and post-intervention design (249). The authors found that the chronic care nursing model resulted in improvements in the opportunity for parents to participate in the plan of care $(28 \% \mathrm{v}$. $100 \%, p=0.019)$ and in the parents' view of the relationship with the ICU staff $(57 \%$ v. $100 \%$, $\mathrm{p}=0.008$ ), based on surveys among 33 parents. An observational study of 292 pediatric ICU patients of a single unit, calculated an index of continuity of nursing care based on the ratio of 
the total number of nurses assigned to the patient over each week. The authors examined the association between this index and ICU length of stay, adjusting for patient characteristics such as severity of illness, and finding that patients with the highest quartile of discontinuity had shorter ICU stays as compared to those with the highest quartile of continuity (Hazard Ratio $=0.12,95 \% \mathrm{Cl}=0.05-0.31$ ). This finding was unexpected and may be due to confounding by unmeasured patient characteristics (247).

Quality of evidence is low due to study design limitations and inconsistency of results. These two studies all have limited generalizability as they were each conducted within a single ICU. The studies also did not show a consistent trend towards or away from improvements in the selected outcomes with increased continuity. There is likely low risk associated with interventions increasing staffing, but it is difficult to assess the overall cost or burden associated with these interventions.

No recommendation can be made due to lack of supporting evidence.

Further research is needed to examine the effect of nurse and physician staffing consistency on family-centered outcomes.

\section{PICO Question 5.7:}

Among family members of ICU patients, does providing a surface for sleep improve family psychological symptoms, quality of life, satisfaction with care, caregiver burden, family or clinician conflict, satisfaction with communication, self-efficacy, trust in clinicians, conferences, quality of communication, or time to DNR?

\section{Evidence Summary:}

Multiple studies find striking levels of sleep-deprivation and sleep-associated morbidity among family members in the ICU. These observational studies utilized both qualitative (251$254)$ and validated, objective measurements $(238,239,255-257)$. The need for access to a location for sleep is a theme expressed by families of the critically ill (252). Indirect evidence informs us that provision of facilities for napping and allowing on-demand naps increases sleep and reduces fatigue among medical staff on call in the hospital $(255-258,258)$. Napping in a bed reduces driving errors (259) and also relieves pain hypersensitivity resulting from sleep deprivation (260).

Families in the ICU suffer significant sleep deprivation and related morbidity. Napping increases daily sleep duration, reduces fatigue, reduces errors, and reduces pain (in non-family, non-ICU populations). Provision of a surface for napping may reduce morbidity among family members, though no studies have been conducted to evaluate the effect of providing a sleep surface on family health. There is a cost to purchasing or building sleep surfaces/space. Given that families desire presence, and there is clear evidence of harm (sleep deprivation) the risk benefit analysis suggests that a sleep surface for families should be provided. The quality of evidence is collectively very low.

\section{Recommendation:}

We suggest that family sleep be considered and families are provided a sleep surface to reduce the effects of sleep deprivation. (2D)

Further research is indicated to determine outcomes related to provision of a family sleep surface, sleep space, napping, and/or light therapy and sleep in the ICU environment. 


\section{Work tools}

Clinicians and healthcare organizations may use these strategies to develop individualized interventions and programs to improve family-centered care in their own ICUs. Tools to enhance translation of the research highlighted in these guidelines into clinical practice are also provided on the Society of Critical Care Medicine (SCCM) website (www.sccm.org) and as an electronic supplement. Work tools supporting these guidelines are also available as electronic supplement Appendix E. Additional work tools are under construction and as available will be posted to the sccm.org website. Finally, a gap analysis tool is being developed by SCCM to support translation of recommendations into practice. Electronic supplement table 6 is also offered as a starting point. Recommendations in Electronic supplement table 6 are summarized in order of ranked importance of outcomes. Organizations and clinicians may prioritize staged implementation based upon the importance of outcomes, perceived barriers and resources at hand.

\section{Limitations}

These guidelines have important limitations that should be acknowledged. First, all 23 recommendations in these guidelines were weak recommendations, reflecting the relatively low quality of evidence regarding interventions to improve family-centered care. The fact that all recommendations were weak highlights the newness of this field of research and the importance of future research to identify the most effective interventions to improve this important aspect of ICU care. Future research is needed that examines the benefits and risks of family presence and participation in ICU settings for patients, families, staff, and clinicians. Quantifying the benefits of partnership with families and the impact on patient outcomes is also needed. Furthermore, research in this area will require additional work to develop, validate, and disseminate rigorous family-centered outcome measures incorporating diverse domains such as satisfaction with care, quality of communication, and quality of decision-making. Second, although we used the qualitative literature to support the generation of topics important to patients and families, and validated topics and importance of outcomes through survivors and families, our pool of patient and family informants was small. Future guidelines writing teams should consider recruiting key patient or family members as co-authors. Furthermore, guidelines writing teams should consider implementing focus groups or key informant interviews serially to validate the progress of the writing team over time. Fourth, although we identify 23 recommendations that we suggest ICUs consider implementing, we found no comparative effectiveness studies that can help identify the most effective interventions and it would be impractical to try to implement all 23 recommendations in a single ICU. Therefore, it will be important that the clinicians and administrators in individual ICUs identify a strategy for staging implementation according to priorities and available resources. Finally, because of the low level of evidence, it is premature to create accreditation or reimbursement standards from the recommendations. However, we offer the suggested recommendations as best known practice given the evidence available today.

\section{Summary}

The primary goal of these guidelines is to identify best practices for family-centered care in the ICU based on existing evidence. These guidelines were developed using a rigorous, objective, and transparent assessment of the relevant published evidence with use of the GRADE methodology. After a systematic review of the literature, the recommendations were developed by taking into consideration not only the quality of the evidence, but also important clinical outcomes and the values and preferences of diverse ICU stakeholders, including patients and family members. The recommendations provide a summary of best practices as identified by existing evidence to form internal policy related to supporting families in the ICU. 
Communication, clinician and family training, family presence, involvement and engagement, provision of consultative resources and environmental and organizational processes are all elements to consider when building an optimal program of family-centered care in the ICU. 
Electronic Table 1: Themes from Qualitative Literature

\begin{tabular}{|l|l|}
\hline Patient Family Themes & Clinician Themes \\
\hline Communication & Communication \\
\hline Presence & Presence \\
\hline Relationship based care & Relationship based care \\
\hline Adaptation/Sensemaking & Adaptation/Sensemaking \\
\hline Operational/Organizational & Operational/Organizational \\
\hline End of life care & End of life care \\
\hline Environment & Environment \\
\hline Individualized care & Individualized care \\
\hline Maintaining Family Integrity & Staff Consequences \\
\hline
\end{tabular}


Electronic Supplement Table 2: Outcomes of interest and importance. 7-10 high, 4-6 moderate, 0-3 low.

\begin{tabular}{|c|c|c|}
\hline OUTCOME & $\begin{array}{l}\text { AVERAGE CLINICIAN RATING } \\
(0-10) \\
\text { (Highest rating most important) } \\
(\mathrm{n}=28)\end{array}$ & $\begin{array}{c}\text { AVERAGE FAMILY RATING } \\
(0-10)(n=7) \\
\text { (Highest rating most important) } \\
(n=7)\end{array}$ \\
\hline $\begin{array}{l}\text { Family Psychological Symptoms (Depression, } \\
\text { Anxiety, PTSD, Prolonged/complicated grief, } \\
\text { Fatigue, PICS) }\end{array}$ & 9.6 & 9.3 \\
\hline Family Quality of Life & 8.2 & 9.1 \\
\hline Family Quality of Dying/Ratings of Dying & 8.2 & 8.4 \\
\hline Caregiver (family) Burden & 8.0 & 8.7 \\
\hline Family Decisional Regret & 7.9 & 8.4 \\
\hline Quality of Communication or Family Conference & 7.7 & 9.3 \\
\hline Family Trust in Clinicians & 7.7 & 9.3 \\
\hline $\begin{array}{l}\text { Family Conferences }(\# / \% \text { receiving conferences, } \\
\text { Time to family conferences }\end{array}$ & 7.6 & 9.0 \\
\hline Family Impact - integrity (Divorce rates, Bonding) & 7.4 & 7.1 \\
\hline Family Satisfaction with Care & 7.3 & 9.4 \\
\hline Family Satisfaction with Communication & 7.3 & 9.1 \\
\hline Family Self-Efficacy & 6.9 & 8.7 \\
\hline Family or Clinician Conflict & 6.70 & 8.0 \\
\hline Clinician Quality of Dying/Rating of Dying & 6.3 & 7.9 \\
\hline $\begin{array}{l}\text { ICU and Hospital Utilization (ICU LOS, ICU costs, } \\
\text { Hospital LOS, Hospital costs, Intensity of care, } \\
\text { TISS) }\end{array}$ & 6.2 & 7.7 \\
\hline Clinician Self-Efficacy & 5.6 & 9.0 \\
\hline $\begin{array}{l}\text { Clinician Psychological Symptoms (Depression, } \\
\text { Anxiety, PTSD, Burnout, Compassion Fatigue, } \\
\text { Moral Distress) }\end{array}$ & 5.5 & 9.0 \\
\hline Time to DNR Order & 5.2 & 6.6 \\
\hline Clinician Job Satisfaction & 5.0 & 8.7 \\
\hline Quality of Teaching & 5.0 & 9.1 \\
\hline Clinician Retention or Intent to Leave Job & 4.6 & 7.6 \\
\hline
\end{tabular}




\begin{tabular}{|l|c|c|}
\hline & & \\
\hline Clinician Time & 4.3 & 7.7 \\
\hline & & 8.7 \\
\hline Adherence to Policy/Protocols & 4.1 & 7.1 \\
\hline Clinician fear of litigation & & 2.8 \\
\hline
\end{tabular}




\section{Electronic Supplement Table 3: Voting Results}

\begin{tabular}{|c|c|c|c|}
\hline \multicolumn{4}{|c|}{ Family Centered-Care Guideline Voting Summary } \\
\hline \multicolumn{4}{|l|}{ Family Presence } \\
\hline \multicolumn{4}{|c|}{ Question 1.1 In the critical care environment, does open flexible visiting hours affect family satisfaction? } \\
\hline $\begin{array}{l}\text { Quality of } \\
\text { Evidence }\end{array}$ & Very Low & & \\
\hline Recommendation & \multicolumn{3}{|c|}{$\begin{array}{l}\text { We suggest that, given the value family members place on presence, dissatisfaction } \\
\text { associated with restricted presence and benefit of engagement associated with presence, } \\
\text { We suggest that family members of critically ill patients be offered open flexible visiting that } \\
\text { meets their needs while supporting staff through the stress imposed by family presence }\end{array}$} \\
\hline $\begin{array}{l}\text { Strength of } \\
\text { Recommendation }\end{array}$ & \multicolumn{3}{|c|}{ Weak Recommendation } \\
\hline Task Force Voting & Agree & Disagree & Abstain \\
\hline Votes & 24 & 1 & 2 \\
\hline Percentages & $88 \%$ & & \\
\hline
\end{tabular}

Question 1.2 In the critical care or emergency department environment, does family presence during interdisciplinary team rounds affect: Family psychological symptoms, Family trust in clinician, Family satisfaction with and preferences for care, Family satisfaction with and preferences for communication, Family or clinician conflict, Quality of teaching, Family participation in rounds, Degree of shared decision-making (as a direct result of family participation), Family knowledge?

\begin{tabular}{|c|c|c|c|}
\hline $\begin{array}{l}\text { Quality of } \\
\text { Evidence }\end{array}$ & $\begin{array}{l}\text { Low } \\
\text { Quality }\end{array}$ & & \\
\hline Recommendation & \multicolumn{3}{|c|}{$\begin{array}{l}\text { We suggest that family members of critically ill patients be offered the option of participating } \\
\text { in interdisciplinary team rounds, but that further research is needed to understand potential } \\
\text { benefits and burdens and long-term effects on family outcomes. }\end{array}$} \\
\hline $\begin{array}{l}\text { Strength of } \\
\text { Recommendation }\end{array}$ & \multicolumn{3}{|c|}{ Weak Recommendation } \\
\hline Task Force Voting & Agree & Disagree & Abstain \\
\hline Votes & 26 & & 1 \\
\hline Percentages & $96 \%$ & & \\
\hline \multicolumn{4}{|c|}{$\begin{array}{l}\text { Question } 1.3 \text { In the critical care or emergency department environment, does family presence during resuscitation affect: } \\
\text { Family psychological symptoms, caregiver burden, family trust in clinician, family satisfaction with care, family satisfaction } \\
\text { with communication, family or clinician conflict? }\end{array}$} \\
\hline $\begin{array}{l}\text { Quality of } \\
\text { Evidence }\end{array}$ & $\begin{array}{l}\text { Low } \\
\text { Quality }\end{array}$ & & \\
\hline Recommendation & \multicolumn{3}{|c|}{$\begin{array}{l}\text { We suggest that family members of critically ill patients be offered the option of being } \\
\text { present during resuscitation efforts. }\end{array}$} \\
\hline $\begin{array}{l}\text { Strength of } \\
\text { Recommendation }\end{array}$ & \multicolumn{3}{|c|}{ Weak Recommendation } \\
\hline
\end{tabular}




\begin{tabular}{|c|c|c|c|}
\hline Task Force Voting & Agree & Disagree & Abstain \\
\hline Votes & 26 & 1 & \\
\hline Percentages & $96 \%$ & & \\
\hline \multicolumn{4}{|l|}{ Family Support } \\
\hline \multicolumn{4}{|c|}{$\begin{array}{l}\text { Question 2.1 Amongst families of ICU patients, does teaching family members to participate in patient care affect: Family } \\
\text { satisfaction with care, Family self-efficacy, Time to DNR order? }\end{array}$} \\
\hline $\begin{array}{l}\text { Quality of } \\
\text { Evidence }\end{array}$ & Moderate & & \\
\hline Recommendation & \multicolumn{3}{|c|}{$\begin{array}{l}\text { We suggest that family members of critically ill patients be offered the option to be taught } \\
\text { how to assist with the care of their loved ones. }\end{array}$} \\
\hline $\begin{array}{l}\text { Strength of } \\
\text { Recommendation }\end{array}$ & \multicolumn{3}{|c|}{ Weak Recommendation } \\
\hline Task Force Voting & Agree & Disagree & Abstain \\
\hline Votes & 27 & & \\
\hline Percentages & $100 \%$ & & \\
\hline \multicolumn{4}{|c|}{$\begin{array}{l}\text { Question } 2.2 \text { Amongst family members of ICU patients do training/education programs for family members affect: Family } \\
\text { psychological symptoms; Family stress; Family satisfaction; reduce health care costs; Family self-efficacy; and reduce hospital } \\
\text { length of stay and costs? }\end{array}$} \\
\hline $\begin{array}{l}\text { Quality of } \\
\text { Evidence }\end{array}$ & Low & & \\
\hline Recommendation & \multicolumn{3}{|c|}{$\begin{array}{l}\text { We suggest that family education programs be included as part of clinical care as these } \\
\text { programs have demonstrated beneficial effects for family members in the ICU. }\end{array}$} \\
\hline $\begin{array}{l}\text { Strength of } \\
\text { Recommendation }\end{array}$ & \multicolumn{3}{|c|}{ Weak Recommendation } \\
\hline Task Force Voting & Agree & Disagree & Abstain \\
\hline Votes & 24 & 2 & 2 \\
\hline Percentages & $88 \%$ & & \\
\hline \multicolumn{4}{|c|}{$\begin{array}{l}\text { Question 2.3 Amongst family members of ICU patients, does provision of family support such as "Date night" or family respite } \\
\text { or family peer-to-peer support affect: family psychological symptoms like PTSD, family satisfaction with care? }\end{array}$} \\
\hline $\begin{array}{l}\text { Quality of } \\
\text { Evidence }\end{array}$ & Very Low & & \\
\hline Recommendation & \multicolumn{3}{|c|}{$\begin{array}{l}\text { We suggest peer-to-peer support be implemented to improve family satisfaction, parental } \\
\text { stress and depression. It is not known whether peer-to-peer support would be effective in } \\
\text { the adult population }\end{array}$} \\
\hline $\begin{array}{l}\text { Strength of } \\
\text { Recommendation }\end{array}$ & \multicolumn{3}{|c|}{ Weak Recommendation } \\
\hline Task Force Voting & Agree & Disagree & Abstain \\
\hline
\end{tabular}




\begin{tabular}{|c|c|c|c|}
\hline Votes & 27 & & 1 \\
\hline Percentages & $100 \%$ & & \\
\hline \multicolumn{4}{|c|}{$\begin{array}{l}\text { Question 2.4 Do written materials such as pamphlets, education materials, and bereavement materials targeting ICU family } \\
\text { members improve outcomes compared to usual care? }\end{array}$} \\
\hline $\begin{array}{l}\text { Quality of } \\
\text { Evidence }\end{array}$ & $\begin{array}{l}\text { Low } \\
\text { Quality }\end{array}$ & & \\
\hline Recommendation & \multicolumn{3}{|c|}{$\begin{array}{l}\text { We suggest that ICUs provide family with information leaflets that give information about } \\
\text { the ICU setting }\end{array}$} \\
\hline $\begin{array}{l}\text { Strength of } \\
\text { Recommendation }\end{array}$ & \multicolumn{3}{|c|}{ Weak Recommendation } \\
\hline Task Force Voting & Agree & Disagree & Abstain \\
\hline Votes & 25 & & 2 \\
\hline Percentages & $92 \%$ & & \\
\hline \multicolumn{4}{|c|}{$\begin{array}{l}\text { Question } 2.5 \text { Among family members of ICU patients does an ICU diary program improve/affect psychological symptoms } \\
\text { (PTSD, Anxiety, Depression)? }\end{array}$} \\
\hline Quality of Evidence & $\begin{array}{l}\text { Low } \\
\text { Quality }\end{array}$ & & \\
\hline Recommendation & \multicolumn{3}{|c|}{ We suggest that ICU diaries be implemented in ICUs } \\
\hline $\begin{array}{l}\text { Strength of } \\
\text { Recommendation }\end{array}$ & \multicolumn{3}{|c|}{ Weak Recommendation } \\
\hline Task Force Voting & Agree & Disagree & Abstain \\
\hline Votes & 25 & 2 & \\
\hline Percentages & $92 \%$ & & \\
\hline \multicolumn{4}{|c|}{$\begin{array}{l}\text { Question } 2.6 \text { In the ICU environment, do decision support tools for families or shared decision making itself improve/affect } \\
\text { communication, cost or length of stay? }\end{array}$} \\
\hline $\begin{array}{l}\text { Quality of } \\
\text { Evidence }\end{array}$ & Very Low & & \\
\hline Recommendation & \multicolumn{3}{|c|}{$\begin{array}{l}\text { We suggest that validated decision support tools for family members be implemented in the } \\
\text { ICU setting when relevant validated tools exist. }\end{array}$} \\
\hline $\begin{array}{l}\text { Strength of } \\
\text { Recommendation }\end{array}$ & \multicolumn{3}{|c|}{ Weak Recommendation } \\
\hline Task Force Voting & Agree & Disagree & Abstain \\
\hline Votes & 23 & 3 & 1 \\
\hline Percentages & $85 \%$ & & \\
\hline \multicolumn{4}{|c|}{$\begin{array}{l}\text { Question } 2.6 \text { In the ICU environment, do clinician support tools targeting family support or primary palliative care such as } \\
\text { checklists, worksheets, mnemonics improve psychological distress or communication compared to usual care? }\end{array}$} \\
\hline $\begin{array}{l}\text { Quality of } \\
\text { Evidence }\end{array}$ & $\begin{array}{l}\text { Low } \\
\text { Quality }\end{array}$ & & \\
\hline Recommendation & \multicolumn{3}{|c|}{$\begin{array}{l}\text { We suggest that, among surrogates of ICU patients who are deemed by a clinician to have a } \\
\text { poor prognosis, clinicians use clinician support tools, such as the use of the mnemonic VALUE } \\
\text { during family conferences, to facilitate clinician-family communication }\end{array}$} \\
\hline
\end{tabular}




\begin{tabular}{|c|c|c|c|c|}
\hline $\begin{array}{l}\text { Strength of } \\
\text { Recommendation }\end{array}$ & \multicolumn{4}{|c|}{ Weak Recommendation } \\
\hline Task Force Voting & Agree & Disagree & \multicolumn{2}{|l|}{ Abstain } \\
\hline Votes & 25 & 1 & \multicolumn{2}{|l|}{1} \\
\hline Percentages & $92 \%$ & & \\
\hline Task Force Voting & Agree & Disagree & \multicolumn{2}{|l|}{ Abstain } \\
\hline Votes & 23 & 3 & \multicolumn{2}{|l|}{1} \\
\hline Percentages & $85 \%$ & & & \\
\hline \multicolumn{5}{|l|}{ Communication } \\
\hline \multicolumn{5}{|c|}{ Question 2.1.a In the ICU setting, do routine family conferences improve/affect patient ICU length of stay (LOS)? } \\
\hline $\begin{array}{l}\text { Quality of } \\
\text { Evidence }\end{array}$ & $\begin{array}{l}\text { Low } \\
\text { Quality }\end{array}$ & & & \\
\hline Recommendation & \multicolumn{4}{|c|}{$\begin{array}{l}\text { We suggest that routine interdisciplinary family conferences should be used in ICU to reduce length of } \\
\text { stay for patients who die in the ICU. }\end{array}$} \\
\hline $\begin{array}{l}\text { Strength of } \\
\text { Recommendation }\end{array}$ & \multicolumn{4}{|c|}{ Weak Recommendation } \\
\hline Task Force Voting & Agree & Disagree & Abstain & Comments \\
\hline Votes & 20 & 2 & 5 & \\
\hline Percentages & $74 \%$ & & & \\
\hline \multicolumn{5}{|c|}{$\begin{array}{l}\text { Question 2.1.b. In the ICU setting, do routine family conferences improve/affect family satisfaction with communication or } \\
\text { care or quality of communication? }\end{array}$} \\
\hline $\begin{array}{l}\text { Quality of } \\
\text { Evidence }\end{array}$ & $\begin{array}{l}\text { Low } \\
\text { quality }\end{array}$ & & & \\
\hline Recommendation & \multicolumn{4}{|c|}{$\begin{array}{l}\text { We suggest that certain, specific communication patterns (e.g. more family and less clinician } \\
\text { speech, use of empathic statements and assurance/support statements with families) can be } \\
\text { used in communication with family members to improve family satisfaction }\end{array}$} \\
\hline $\begin{array}{l}\text { Strength of } \\
\text { Recommendation }\end{array}$ & \multicolumn{4}{|c|}{ Weak Recommendation } \\
\hline Task Force Voting & Agree & Disagree & \multicolumn{2}{|c|}{ Abstain } \\
\hline Votes & 25 & 2 & & \\
\hline Percentages & $92 \%$ & & & \\
\hline \multicolumn{5}{|c|}{$\begin{array}{l}\text { Question 2.1c In the ICU setting, do routine family conferences improve family trust in clinicians, decrease } \\
\text { family/clinician conflict; or affect intensity of , or time devoted to, care? }\end{array}$} \\
\hline $\begin{array}{l}\text { Quality of } \\
\text { Evidence }\end{array}$ & $\begin{array}{l}\text { Low } \\
\text { Quality }\end{array}$ & & & \\
\hline Recommendation & \multicolumn{4}{|c|}{$\begin{array}{l}\text { We suggest that routine interdisciplinary family conferences should be used in ICU to } \\
\text { improve family trust and reduce conflict between clinicians and family members. }\end{array}$} \\
\hline
\end{tabular}




\begin{tabular}{|c|c|c|c|}
\hline $\begin{array}{l}\text { Strength of } \\
\text { Recommendation }\end{array}$ & \multicolumn{3}{|c|}{ Weak Recommendation } \\
\hline Task Force Voting & Agree & Disagree & Abstain \\
\hline Votes & 26 & & 1 \\
\hline Percentages & $96 \%$ & & \\
\hline \multicolumn{4}{|c|}{$\begin{array}{l}\text { Question 2.2. Amongst healthcare clinicians in the ICU, do specific communication techniques such as active listening, } \\
\text { empathy and empathic statements, provision of hope, bedside caring behaviors including touch, provision of supportive } \\
\text { comments, language translation or cultural mediation affect family psychological symptoms, family satisfaction with care, } \\
\text { communication or decision-making, physician-family conflict, or ICU utilization (length of stay)? }\end{array}$} \\
\hline $\begin{array}{l}\text { Quality of } \\
\text { Evidence }\end{array}$ & $\begin{array}{l}\text { Low } \\
\text { Quality }\end{array}$ & & \\
\hline Recommendation & \multicolumn{3}{|c|}{$\begin{array}{l}\text { We suggest that health care clinicians in the ICU utilize strategies included in the VALUE } \\
\text { mnemonic when engaging in communication with family members, specifically active } \\
\text { listening, expressions of empathy, making supportive statements around non-abandonment } \\
\text { and decision-making. In addition, we suggest that family members of critically ill patients be } \\
\text { offered a written brochure. }\end{array}$} \\
\hline $\begin{array}{l}\text { Strength of } \\
\text { Recommendation }\end{array}$ & \multicolumn{3}{|c|}{ Weak Recommendation } \\
\hline Task Force Voting & Agree & Disagree & Abstain \\
\hline Votes & 25 & 1 & 1 \\
\hline Percentages & $92 \%$ & & \\
\hline \multicolumn{4}{|c|}{$\begin{array}{l}\text { Question 2.3a In the ICU environment, do communication training programs for clinicians such as education or simulation } \\
\text { improve/affect: Family Psychological Symptoms ( } 1 \text { study) Family Quality of Dying ( } 1 \text { study) Quality of Communication (5 } \\
\text { studies) Family Satisfaction with Communication ( } 2 \text { studies) Clinician Self-Efficacy (10 studies)• Clinician Psychological } \\
\text { Symptoms ( } 2 \text { studies)? }\end{array}$} \\
\hline $\begin{array}{l}\text { Quality of } \\
\text { Evidence }\end{array}$ & Very Low & & \\
\hline Recommendation & \multicolumn{3}{|c|}{$\begin{array}{l}\text { Based on existing evidence of patient and family burden associated with poor } \\
\text { communication, as well as improved clinician-reported skills and comfort following } \\
\text { communication training, we suggest that ICU clinicians receive family-centered } \\
\text { communication training as one element of an overall well-rounded critical care training } \\
\text { curriculum and ongoing education. }\end{array}$} \\
\hline $\begin{array}{l}\text { Strength of } \\
\text { Recommendation }\end{array}$ & \multicolumn{3}{|c|}{ Weak Recommendation } \\
\hline Task Force Voting & Agree & Disagree & Abstain \\
\hline Votes & 27 & & 1 \\
\hline Percentages & $100 \%$ & & \\
\hline \multicolumn{4}{|c|}{$\begin{array}{l}\text { Question 2.3b In the ICU environment, do communication training programs for clinicians such as education or simulation } \\
\text { improve/affect: Family Psychological Symptoms ( } 1 \text { study) Family Quality of Dying ( } 1 \text { study) Quality of Communication (5 } \\
\text { studies) Family Satisfaction with Communication ( } 2 \text { studies) Clinician Self-Efficacy (10 studies) Clinician Psychological } \\
\text { Symptoms ( } 2 \text { studies)? }\end{array}$} \\
\hline
\end{tabular}




\begin{tabular}{|c|c|c|c|}
\hline $\begin{array}{l}\text { Quality of } \\
\text { Evidence }\end{array}$ & Very Low & & \\
\hline Recommendation & \multicolumn{3}{|c|}{$\begin{array}{l}\text { No Recommendation can be made to suggest the use of any of the specific communication } \\
\text { training programs that have been evaluated based on existing evidence. }\end{array}$} \\
\hline $\begin{array}{l}\text { Strength of } \\
\text { Recommendation }\end{array}$ & \multicolumn{3}{|c|}{ No Recommendation } \\
\hline Task Force Voting & Agree & Disagree & Abstain \\
\hline Votes & 25 & 2 & 1 \\
\hline Percentages & $92 \%$ & & \\
\hline \multicolumn{4}{|l|}{ Consultations } \\
\hline \multicolumn{4}{|c|}{$\begin{array}{l}\text { Question 1.1 Among family members of ICU patients does a palliative care consultation impact ICU and hospital } \\
\text { utilization? }\end{array}$} \\
\hline $\begin{array}{l}\text { Quality of } \\
\text { Evidence }\end{array}$ & $\begin{array}{l}\text { Low } \\
\text { Quality }\end{array}$ & & \\
\hline Recommendation & \multicolumn{3}{|c|}{$\begin{array}{l}\text { We suggest that proactive palliative care consultation be considered to decrease ICU and } \\
\text { hospital length among selected critically ill patients }\end{array}$} \\
\hline $\begin{array}{l}\text { Strength of } \\
\text { Recommendation }\end{array}$ & \multicolumn{3}{|c|}{ Weak Recommendation } \\
\hline Task Force Voting & Agree & Disagree & Abstain \\
\hline Votes & 26 & 1 & \\
\hline Percentages & $96 \%$ & & \\
\hline \multicolumn{4}{|c|}{$\begin{array}{l}\text { Question 1.2a Among family members of ICU patients does ethics consultation impact family satisfaction, ICU } \\
\text { or hospital length of stay? }\end{array}$} \\
\hline $\begin{array}{l}\text { Quality of } \\
\text { Evidence }\end{array}$ & $\begin{array}{l}\text { Low } \\
\text { Quality }\end{array}$ & & \\
\hline Recommendation & \multicolumn{3}{|c|}{$\begin{array}{l}\text { We suggest that ethics consultation, particularly in reaction to a conflict about goals of care } \\
\text { for an ICU patient, may decrease ICU and hospital length of stay among selected critically ill } \\
\text { patients }\end{array}$} \\
\hline $\begin{array}{l}\text { Strength of } \\
\text { Recommendation }\end{array}$ & \multicolumn{3}{|c|}{ Weak Recommendation } \\
\hline Task Force Voting & Agree & Disagree & Abstain \\
\hline Votes & 24 & 2 & 2 \\
\hline Percentages & $88 \%$ & & \\
\hline \multicolumn{4}{|c|}{$\begin{array}{l}\text { Question } 1.2 b \text { Among family members of ICU patients does ethics consultation impact family satisfaction, ICU } \\
\text { or hospital length of stay? }\end{array}$} \\
\hline $\begin{array}{l}\text { Quality of } \\
\text { Evidence }\end{array}$ & $\begin{array}{l}\text { Low } \\
\text { Quality }\end{array}$ & & \\
\hline Recommendation & \multicolumn{3}{|c|}{$\begin{array}{l}\text { No recommendation can be made about using ethics consultation with the goal of increasing } \\
\text { family satisfaction }\end{array}$} \\
\hline $\begin{array}{l}\text { Strength of } \\
\text { Recommendation }\end{array}$ & \multicolumn{3}{|c|}{ No Recommendation } \\
\hline Task Force Voting & Agree & Disagree & Abstain \\
\hline
\end{tabular}




\begin{tabular}{|c|c|c|c|}
\hline Votes & 26 & 1 & 1 \\
\hline Percentages & $96 \%$ & & \\
\hline \multicolumn{4}{|c|}{ Question 1.3 Among family members of ICU patients does a psychologist improve/affect family outcomes? } \\
\hline $\begin{array}{l}\text { Quality of } \\
\text { Evidence }\end{array}$ & $\begin{array}{l}\text { Low } \\
\text { Quality }\end{array}$ & & \\
\hline Recommendation & \multicolumn{3}{|c|}{$\begin{array}{l}\text { We suggest a psychologist's intervention to specifically incorporate a multimodal CBT-based } \\
\text { approach to improve outcomes in mothers of pre-term babies admitted to the NICU based } \\
\text { on a single center study }\end{array}$} \\
\hline $\begin{array}{l}\text { Strength of } \\
\text { Recommendation }\end{array}$ & \multicolumn{3}{|c|}{ Weak Recommendation } \\
\hline Task Force Voting & Agree & Disagree & Abstain \\
\hline Votes & 26 & & 1 \\
\hline Percentages & $96 \%$ & & \\
\hline $\begin{array}{l}\text { Quality of } \\
\text { Evidence }\end{array}$ & $\begin{array}{l}\text { Low } \\
\text { Quality }\end{array}$ & & \\
\hline \multicolumn{4}{|c|}{ Question 1.3b Among family members of ICU patients does a psychologist improve/ affect family outcomes? } \\
\hline $\begin{array}{l}\text { Quality of } \\
\text { Evidence }\end{array}$ & $\begin{array}{l}\text { Low } \\
\text { Quality }\end{array}$ & & \\
\hline Recommendation & \multicolumn{3}{|c|}{$\begin{array}{l}\text { We suggest that targeted video and reading materials be considered in the context of } \\
\text { psychological support provided to mothers of pre-term babies admitted to the ICU, based on } \\
\text { a single study }\end{array}$} \\
\hline $\begin{array}{l}\text { Strength of } \\
\text { Recommendation }\end{array}$ & \multicolumn{3}{|c|}{ Weak Recommendation } \\
\hline Task Force Voting & Agree & Disagree & Abstain \\
\hline Votes & 25 & 1 & 2 \\
\hline Percentages & $92 \%$ & & \\
\hline \multicolumn{4}{|c|}{ Question 1.4 Among family members of ICU patients does a social work consultation impact family satisfaction? } \\
\hline $\begin{array}{l}\text { Quality of } \\
\text { Evidence }\end{array}$ & Very Low & & \\
\hline Recommendation & \multicolumn{3}{|c|}{$\begin{array}{l}\text { We suggest social workers be included within an interdisciplinary team to participate in } \\
\text { family meetings and improve family satisfaction }\end{array}$} \\
\hline $\begin{array}{l}\text { Strength of } \\
\text { Recommendation }\end{array}$ & \multicolumn{3}{|c|}{ Weak Recommendation } \\
\hline Task Force Voting & Agree & Disagree & Abstain \\
\hline Votes & 26 & 1 & \\
\hline Percentages & $96 \%$ & & \\
\hline
\end{tabular}




\begin{tabular}{|c|c|c|c|c|}
\hline $\begin{array}{l}\text { Quality of } \\
\text { Evidence }\end{array}$ & Very Low & & & \\
\hline Recommendation & \multicolumn{4}{|c|}{$\begin{array}{l}\text { We suggest that patient/family navigators be assigned to families throughout the ICU stay to } \\
\text { improve family satisfaction with communication, family psychological symptoms, and reduce } \\
\text { length of ICU stay and costs of care. }\end{array}$} \\
\hline $\begin{array}{l}\text { Strength of } \\
\text { Recommendation }\end{array}$ & \multicolumn{4}{|c|}{ Weak Recommendation } \\
\hline Task Force Voting & Agree & Disagree & \multicolumn{2}{|l|}{ Abstain } \\
\hline Votes & 25 & 1 & \multicolumn{2}{|l|}{1} \\
\hline Percentages & $92 \%$ & & & \\
\hline \multicolumn{5}{|c|}{ Question 1.5.b Among family members of ICU patients does a patient navigator improve/affect family satisfaction? } \\
\hline $\begin{array}{l}\text { Quality of } \\
\text { Evidence }\end{array}$ & $\begin{array}{l}\text { Low } \\
\text { Quality }\end{array}$ & & & \\
\hline Recommendation & \multicolumn{4}{|c|}{$\begin{array}{l}\text { We suggest that patient/family navigators be considered in the ICU to improve family } \\
\text { satisfaction with physician communication }\end{array}$} \\
\hline $\begin{array}{l}\text { Strength of } \\
\text { Recommendation }\end{array}$ & \multicolumn{4}{|c|}{ Weak Recommendation } \\
\hline Task Force Voting & Agree & Disagree & Abstain & Comments \\
\hline Votes & 22 & 2 & 3 & \\
\hline Percentages & $81 \%$ & & & \\
\hline \multicolumn{5}{|c|}{$\begin{array}{l}\text { Question 1.5.c Among family members of ICU patients does a patient/family navigator improve/affect Family or Clinician } \\
\text { Conflict? }\end{array}$} \\
\hline $\begin{array}{l}\text { Quality of } \\
\text { Evidence }\end{array}$ & Very Low & & & \\
\hline Recommendation & \multicolumn{4}{|c|}{ No recommendation can be made due to lack of sufficient evidence } \\
\hline $\begin{array}{l}\text { Strength of } \\
\text { Recommendation }\end{array}$ & \multicolumn{4}{|c|}{ No Recommendation } \\
\hline Task Force Voting & Agree & Disagree & Abstain & Comments \\
\hline Votes & 26 & & 1 & \\
\hline Percentages & $96 \%$ & & & \\
\hline \multicolumn{5}{|c|}{ Question 1.5.d Among family members of ICU patients does a patient/family navigator improve/affect resource utilization? } \\
\hline $\begin{array}{l}\text { Quality of } \\
\text { Evidence }\end{array}$ & Very Low & & & \\
\hline Recommendation & No recomm & endation ca & be made & to lack of sufficient evidence \\
\hline
\end{tabular}




\begin{tabular}{|c|c|c|c|}
\hline $\begin{array}{l}\text { Strength of } \\
\text { Recommendation }\end{array}$ & \multicolumn{3}{|c|}{ No Recommendation } \\
\hline Task Force Voting & Agree & Disagree & Abstain \\
\hline Votes & 25 & 1 & 1 \\
\hline Percentages & $92 \%$ & & \\
\hline \multicolumn{4}{|c|}{$\begin{array}{l}\text { Question 1.6 Among families of ICU patients, does routine consultation of a spiritual care provider, as compared to usual } \\
\text { care, improve outcomes? }\end{array}$} \\
\hline $\begin{array}{l}\text { Quality of } \\
\text { Evidence }\end{array}$ & Very Low & & \\
\hline Recommendation & \multicolumn{3}{|c|}{$\begin{array}{l}\text { Given the consistency of expression of family values for availability of spiritual care, the } \\
\text { accreditation standards, and the results of the observation study we propose that families be } \\
\text { offered spiritual support with a spiritual advisor or pastor. The best method for provision of } \\
\text { spiritual support has not been studied and warrants further investigation }\end{array}$} \\
\hline $\begin{array}{l}\text { Strength of } \\
\text { Recommendation }\end{array}$ & \multicolumn{3}{|c|}{ Weak Recommendation } \\
\hline Task Force Voting & Agree & Disagree & Abstain \\
\hline Votes & 24 & 3 & \\
\hline Percentages & $88 \%$ & & \\
\hline \multicolumn{4}{|c|}{ Organization/Environment } \\
\hline \multicolumn{4}{|c|}{ Question 5.1+74:80 In the ICU environment, do protocols for withdrawing life support improve outcomes? } \\
\hline $\begin{array}{l}\text { Quality of } \\
\text { Evidence }\end{array}$ & $\begin{array}{l}\text { Low } \\
\text { Quality }\end{array}$ & & \\
\hline Recommendation & \multicolumn{3}{|c|}{$\begin{array}{l}\text { We suggest that protocols be implemented to ensure adequate and standardized use of } \\
\text { sedation and analgesia during withdrawal of life support }\end{array}$} \\
\hline $\begin{array}{l}\text { Strength of } \\
\text { Recommendation }\end{array}$ & \multicolumn{3}{|c|}{ Weak Recommendation } \\
\hline \multicolumn{4}{|c|}{$\begin{array}{l}\text { Deleted Question Do executive (hospital leadership) walk rounds improve family-centered outcomes in the } \\
\text { ICU? }\end{array}$} \\
\hline $\begin{array}{l}\text { Quality of } \\
\text { Evidence }\end{array}$ & Very Low & & \\
\hline Recommendation & \multicolumn{3}{|c|}{ No recommendation can be made due to lack of supporting evidence } \\
\hline $\begin{array}{l}\text { Strength of } \\
\text { Recommendation }\end{array}$ & \multicolumn{3}{|c|}{ No Recommendation } \\
\hline Task Force Voting & Agree & Disagree & Abstain \\
\hline Votes & 27 & & 1 \\
\hline Percentages & $100 \%$ & & \\
\hline
\end{tabular}




\begin{tabular}{|c|c|c|c|}
\hline \multicolumn{4}{|c|}{ Question 5.2a Does the inclusion of nurses in ICU communication improve family-centered outcomes? } \\
\hline $\begin{array}{l}\text { Quality of } \\
\text { Evidence }\end{array}$ & Very Low & & \\
\hline Recommendation & \multicolumn{3}{|c|}{$\begin{array}{l}\text { We suggest that specialized nurses be used as part of an overall program to potentially } \\
\text { decrease ICU and hospital length of stay and to improve patient/family perception of the } \\
\text { quality of communication in the ICU }\end{array}$} \\
\hline $\begin{array}{l}\text { Strength of } \\
\text { Recommendation }\end{array}$ & \multicolumn{3}{|c|}{ Weak Recommendation } \\
\hline Task Force Voting & Agree & Disagree & Abstain \\
\hline Votes & 25 & 1 & 2 \\
\hline Percentages & $92 \%$ & & \\
\hline \multicolumn{4}{|c|}{ Question 5.2.b Does the inclusion of nurses in ICU decision making improve family-centered outcomes? } \\
\hline $\begin{array}{l}\text { Quality of } \\
\text { Evidence }\end{array}$ & Very Low & & \\
\hline Recommendation & \multicolumn{3}{|c|}{$\begin{array}{l}\text { No recommendation can be made regarding decision-making due to lack of supporting } \\
\text { evidence }\end{array}$} \\
\hline $\begin{array}{l}\text { Strength of } \\
\text { Recommendation }\end{array}$ & \multicolumn{3}{|c|}{ No Recommendation } \\
\hline Task Force Voting & Agree & Disagree & Abstain \\
\hline Votes & 26 & & 2 \\
\hline Percentages & $96 \%$ & & \\
\hline \multicolumn{4}{|c|}{ Deleted Question: Does consistency in staffing improve family-centered outcomes during critical illness? } \\
\hline $\begin{array}{l}\text { Quality of } \\
\text { Evidence }\end{array}$ & Low & & \\
\hline Recommendation & \multicolumn{3}{|c|}{ No recommendation can be made due to lack of supporting evidence } \\
\hline $\begin{array}{l}\text { Strength of } \\
\text { Recommendation }\end{array}$ & \multicolumn{3}{|c|}{ No Recommendation } \\
\hline Task Force Voting & Agree & Disagree & Abstain \\
\hline Votes & 26 & 1 & 1 \\
\hline Percentages & $96 \%$ & & \\
\hline \multicolumn{4}{|c|}{$\begin{array}{l}\text { Question } 5.3 \text { Does a comprehensive "family-centered care" approach to ICU care improve family-centered outcomes during } \\
\text { critical illness? }\end{array}$} \\
\hline $\begin{array}{l}\text { Quality of } \\
\text { Evidence }\end{array}$ & Very Low & & \\
\hline Recommendation & \multicolumn{3}{|c|}{$\begin{array}{l}\text { We suggest that hospitals consider implementing policies to promote family-centered care in } \\
\text { the ICU to improve family experience }\end{array}$} \\
\hline
\end{tabular}




\begin{tabular}{|c|c|c|c|}
\hline Strength of & \multicolumn{3}{|c|}{ Weak Recommendation } \\
\hline Task Force Voting & Agree & Disagree & Abstain \\
\hline Votes & 27 & & 1 \\
\hline Percentages & $100 \%$ & & \\
\hline \multicolumn{4}{|c|}{$\begin{array}{l}\text { Question } 5.4 \text { In the critical care environment do noise reduction strategies for family members (beyond single rooms for } \\
\text { patients) affect patient/family satisfaction, staff stress or noise? }\end{array}$} \\
\hline $\begin{array}{l}\text { Quality of } \\
\text { Evidence }\end{array}$ & Very Low & & \\
\hline Recommendation & \multicolumn{3}{|c|}{$\begin{array}{l}\text { In the absence of evidence to support specific strategies other than private rooms for noise } \\
\text { reduction, yet given the evidence of harm from noise, we suggest that ICUs implement noise } \\
\text { reduction and environmental hygiene practices. }\end{array}$} \\
\hline $\begin{array}{l}\text { Strength of } \\
\text { Recommendation }\end{array}$ & \multicolumn{3}{|c|}{ Weak Recommendation } \\
\hline Task Force Voting & Agree & Disagree & Abstain \\
\hline Votes & 27 & & 1 \\
\hline Percentages & $100 \%$ & & \\
\hline \multicolumn{4}{|c|}{$\begin{array}{l}\text { Question } 5.4 \text { In the critical care environment do private rooms or space for family members (beyond single rooms for } \\
\text { patients) affect patient/family satisfaction, staff stress or noise? }\end{array}$} \\
\hline $\begin{array}{l}\text { Quality of } \\
\text { Evidence }\end{array}$ & Very Low & & \\
\hline Recommendation & \multicolumn{3}{|c|}{$\begin{array}{l}\text { We suggest the use of private rooms to improve patient/family satisfaction and noise } \\
\text { reduction while managing staff stress imposed with the change from open-bay to private } \\
\text { rooms in the NICU. }\end{array}$} \\
\hline $\begin{array}{l}\text { Strength of } \\
\text { Recommendation }\end{array}$ & \multicolumn{3}{|c|}{ Weak Recommendation } \\
\hline Task Force Voting & Agree & Disagree & Abstain \\
\hline Votes & 25 & 2 & 1 \\
\hline Percentages & $92 \%$ & & \\
\hline \multicolumn{4}{|c|}{$\begin{array}{l}\text { Question } 5.4 \text { In the critical care environment do noise reduction strategies, private rooms or space for family members } \\
\text { (beyond single rooms for patients) affect patient/family satisfaction, staff stress or noise? }\end{array}$} \\
\hline $\begin{array}{l}\text { Quality of } \\
\text { Evidence }\end{array}$ & Very Low & & \\
\hline Recommendation & \multicolumn{3}{|c|}{$\begin{array}{l}\text { No Recommendation can be made for family space. However, it is noted that the } \\
\text { SCCM/ACCM Guidelines for ICU design recommend designing new ICUs with family space } \\
\text { based upon consensus statement (Thompson-4843) }\end{array}$} \\
\hline $\begin{array}{l}\text { Strength of } \\
\text { Recommendation }\end{array}$ & \multicolumn{3}{|c|}{ No Recommendation } \\
\hline
\end{tabular}




\begin{tabular}{|l|l|l|l|}
\cline { 4 - 4 } Task Force Voting & Agree & Disagree & Abstain \\
\hline Votes & 25 & 1 & 2 \\
\hline Percentages & $92 \%$ & & \\
\hline $\begin{array}{l}\text { Question } 5.5 \text { Among family members of ICU patients, does providing a surface or space for sleep improve family } \\
\text { psychological symptoms, quality of life, satisfaction with care, caregiver burden, family or clinician conflict, satisfaction with } \\
\text { communication, self-efficacy, trust in clinicians, conferences, quality of communication, or time to DNR? }\end{array}$ \\
\hline $\begin{array}{l}\text { Recommendation } \\
\text { in close proximity to the patient in order to improve family outcomes }\end{array}$ \\
\hline $\begin{array}{l}\text { Quality of } \\
\text { Evidence }\end{array}$ & Wery suggest that family members or space \\
\hline $\begin{array}{l}\text { Strength of } \\
\text { Recommendation }\end{array}$ & Weak Recommendation \\
\hline Task Force Voting & Agree & Disagree & Abstain \\
\hline Votes & 25 & 2 & \\
\hline Percentages & $92 \%$ & & \\
\hline
\end{tabular}


Appendix A: Developing Definitions of "family" and "family-centered care"

We identified previous published definitions of "family" and "family-centered care" from published guidelines and key documents on family-centered care. A search strategy and handsearch yielded 24 articles, books, and pamphlets offering definitions of "family", "patientcentered care", and/or "family-centered care"; $(8,13,21,61,261-278)$ these definitions were abstracted by a sub-group composed of five panel members. Definitions were summarized in a single document (Electronic supplement tables 4 and 5), circulated to the five sub-group members along with a panel leader (JRC), and discussed. Through an iterative process, proposed definitions were summarized, discussed, eliminated, and modified. The group decided to exclude "patient-centered care" as beyond the scope of the intended review and guidelines. Two potential definitions of "family" and three potential definitions of "family-centered care" were developed and, along with summaries of the discussion surrounding each proposed definition, presented to the entire advisory panel for further development and discussion (Electronic supplement table 6).

Through an online survey, the entire guidelines writing group panel indicated their preferences for the proposed definitions and were given the opportunity to provide alternative definitions. The proposed definitions were also provided to a group of 7 former ICU patients and family members, including three adult survivors of critical illness and four family members of critically ill adults.

Incorporating alternative definitions and refining wording of the proposed definitions, the final definitions were selected and determined to be acceptable by greater than $70 \%$ of the expert panel. These final selected definitions for "family" and "family-centered care" were unanimously deemed appropriate and acceptable by the participating former ICU patients and family members. We define family as:

Family is defined by the patient or, in the case of minors or those without decision making capacity, by their surrogates. In this context, the family may be related or 
unrelated to the patient. They are individuals who provide support and with whom the patient has a significant relationship.

We define family-centered care as:

Family-centered care is an approach to health care that is respectful of and responsive to individual family's needs and values. 
Electronic Supplement Table 4: Abstracted definitions of terms "family" organized by domain

\begin{tabular}{|c|c|}
\hline \multicolumn{2}{|r|}{ "Family" } \\
\hline Domain & Definitions (examples) \\
\hline \multirow{2}{*}{$\begin{array}{l}\text { Family is defined } \\
\text { by the patient } \\
\text { and family. }\end{array}$} & $\begin{array}{l}\text { "Two or more persons who are related in any way-biologically, legally, or } \\
\text { emotionally. Patients and families define their families." } 24\end{array}$ \\
\hline & $\begin{array}{l}\text { "For the purposes of this document, the definition of family published by the } \\
\text { National Consensus Project for Quality Palliative Care is adopted: "Family is } \\
\text { defined by the patient or in the case of minors or those without decision making } \\
\text { capacity by their surrogates. In this context the family may be related or } \\
\text { unrelated to the patient. They are individuals who provide support and with } \\
\text { whom the patient has a significant relationship" }{ }^{6}\end{array}$ \\
\hline $\begin{array}{l}\text { Family is a social } \\
\text { unit. }\end{array}$ & $\begin{array}{l}\text { "The family is a basic social unit having as its nucleus two or more persons, } \\
\text { irrespective of age, in which each of the following conditions are present: } 1 \text {. the } \\
\text { members are related by blood, or marriage, or adoption, or by a contract which } \\
\text { is either explicit or implied; } 2 \text {. the members communicate with each other in } \\
\text { terms of defined social roles such as mother, father, wife, husband, daughter, } \\
\text { son, brother, sister, grandfather, grandmother, uncle, aunt; and 3. they adopt or } \\
\text { create and maintain common customs and traditions." } 19\end{array}$ \\
\hline $\begin{array}{l}\text { Family defies } \\
\text { definition }\end{array}$ & $\begin{array}{l}\text { "We all come from families. Families are big, small, extended, nuclear, } \\
\text { multigenerational, with one parent, two parents and grandparents. We live } \\
\text { under one roof or many. A family can be as temporary as a few weeks, as } \\
\text { permanent as forever. We become part of a family by birth, adoption, marriage, } \\
\text { or from a desire for mutual support. As family members, we nurture, protect, } \\
\text { and influence one another. Families are dynamic and are cultures unto } \\
\text { themselves, with different values and unique ways of realizing dreams. Together, } \\
\text { our families become the source of our rich cultural heritage and spiritual } \\
\text { diversity. Each family has strengths and qualities that flow from individual } \\
\text { members and from the family as a unit. Our families create neighborhoods, } \\
\text { communities, states, and nations." } 2\end{array}$ \\
\hline
\end{tabular}


Electronic Supplement Table 5: Abstracted definitions of terms "family-centered care" organized by domain

\begin{tabular}{|c|c|}
\hline \multicolumn{2}{|r|}{ "Family-centered care" } \\
\hline Domain & Definitions \\
\hline \multirow[t]{2}{*}{$\begin{array}{l}\text { Definition by } \\
\text { tenets }\end{array}$} & $\begin{array}{l}\text { "There are a number of key principles to family-centered care: (1) including } \\
\text { parents and families being treated with dignity and respect; (2) parents having a } \\
\text { right to know about their infant's care and condition and updated information } \\
\text { should be available to them, health care providers prioritizing open } \\
\text { communication and sharing information with parents and families in ways that } \\
\text { are affirming and useful; (3) information-giving being tailored according to } \\
\text { parents' individual preferences for detail and their changing needs; (4) parents } \\
\text { and families being encouraged to participate in their infant's care with the aim of } \\
\text { them developing a sense of confidence, control and growing independence; and } \\
\text { (5) practical and emotional support being provided continuously, through the } \\
\text { care pathway"2 }\end{array}$ \\
\hline & $\begin{array}{l}\text { "In the Institute of Medicine's patient-centered model, a) patients and families } \\
\text { are kept informed and actively involved in medical decisionmaking and self- } \\
\text { management; b) patient care is coordinated and integrated across groups of } \\
\text { healthcare providers; c) healthcare delivery systems provide for the physical } \\
\text { comfort and emotional support of patients and family members; d) healthcare } \\
\text { providers have a clear understanding of patients' concepts of illness and their } \\
\text { cultural beliefs; and e) healthcare providers understand and apply principles of } \\
\text { disease prevention and behavioral change appropriate for diverse populations." }\end{array}$ \\
\hline $\begin{array}{l}\text { Care involving } \\
\text { patient } \\
\text { preferences and } \\
\text { goals }\end{array}$ & $\begin{array}{l}\text { "Family centered-care, which sees patients as embedded within a social } \\
\text { structure and web of relationships, is emerging as a comprehensive ideal for } \\
\text { end-of-life care in the ICU." "Family centered care is based on the values, goals, } \\
\text { and needs of the patient and family, including their understanding of the illness, } \\
\text { prognosis, and treatment options and their expectations and preferences for } \\
\text { treatment and decision making." } 23\end{array}$ \\
\hline $\begin{array}{l}\text { Care involving } \\
\text { compassion, } \\
\text { empathy, and the } \\
\text { whole patient }\end{array}$ & $\begin{array}{l}\text { "Patient-centered encompasses qualities of compassion, empathy, and } \\
\text { responsiveness to the needs, values, and expressed preferences of the individual } \\
\text { patient." } 25\end{array}$ \\
\hline $\begin{array}{l}\text { Care respectful of } \\
\text { cultural } \\
\text { competence }\end{array}$ & $\begin{array}{l}\text { "Patient-centered care responds precisely to each patient's wants, needs, and } \\
\text { preferences. It gives patients abundant opportunities to be informed and } \\
\text { involved in medical decision making, and guides and supports those providing } \\
\text { care in attending to their patients' physical and emotional needs, and } \\
\text { maintaining or improving their quality of life to the extent possible. Patient- } \\
\text { centered care is highly customized and incorporates cultural competence." }{ }^{25}\end{array}$ \\
\hline \multirow[t]{2}{*}{$\begin{array}{l}\text { Care that treats } \\
\text { the patient and } \\
\text { family }\end{array}$} & $\begin{array}{l}\text { "A way of caring for children and their families within health services which } \\
\text { ensures that care is planned around the whole family, not just the individual } \\
\text { child/person, and in which all the family members are recognised as care } \\
\text { recipients"19 }\end{array}$ \\
\hline & $\begin{array}{l}\text { "[Family centered care] is an approach to health care in which: providers } \\
\text { recognize the importance that families paly in ensuring the health and well-being }\end{array}$ \\
\hline
\end{tabular}




\begin{tabular}{|l|l|}
\hline & $\begin{array}{l}\text { of their children; providers support families in their care-giving and decision } \\
\text { making roles; providers have an awareness of the importance of meeting the } \\
\text { psychosocial and developmental needs of children and the role of families in } \\
\text { promoting the health and well -being of their children; 'the philosophies, } \\
\text { principles and practices that put the family at the heart or center of services;"' } 1\end{array}$ \\
\hline $\begin{array}{l}\text { Care that is a } \\
\text { partnership } \\
\text { between } \\
\text { providers and } \\
\text { patients and } \\
\text { families }\end{array}$ & $\begin{array}{l}\text { Collaboration between providers and families and patients in the planning, } \\
\text { delivery and evaluation of care- family-centered care and patient andfamily- } \\
\text { centered care "more explicitly capture the importance of engaging the family } \\
\text { and the patient in a developmentally supportive manner as essential members of } \\
\text { the health care team." }\end{array}$ \\
\hline
\end{tabular}


Electronic Supplement Table 6: Definitions of terms "family" and "family-centered care" presented to entire group

\begin{tabular}{|c|c|}
\hline Term & Definitions \\
\hline \multirow[b]{2}{*}{ Family } & $\begin{array}{l}\text { "Two or more persons who are related in any way-biologically, legally, or } \\
\text { emotionally. Patients and families define their families." } 24\end{array}$ \\
\hline & $\begin{array}{l}\text { "Family is defined by the patient or in the case of minors or those without } \\
\text { decision making capacity by their surrogates. In this context the family may be } \\
\text { related or unrelated to the patient. They are individuals who provide support } \\
\text { and with whom the patient has a significant relationship" } 6\end{array}$ \\
\hline \multirow{3}{*}{$\begin{array}{c}\text { Family-centered } \\
\text { care }\end{array}$} & $\begin{array}{l}\text { Family-centered care is an approach to health care that is respectful of and } \\
\text { responsive to individual family's needs and values. } \\
\text { Derived from }{ }^{25}\end{array}$ \\
\hline & $\begin{array}{l}\text { Family-centered care recognizes that patients are embedded within a social } \\
\text { structure and web of relationships. It is an approach to health care that is } \\
\text { respectful of and responsive to individual family's needs and values. } \\
\text { Derived from }{ }^{23}\end{array}$ \\
\hline & $\begin{array}{l}\text { Family-centered care recognizes that patients are embedded within a social } \\
\text { structure and web of relationships. It is an approach to health care that is } \\
\text { planned around the individual patient as the primary focus, but also views the } \\
\text { patient as part of a family whose members are recognized as care recipients. } \\
\text { Derived from }{ }^{23}\end{array}$ \\
\hline
\end{tabular}


Appendix B: Standard GRADE methodology used

The Refworks $\circledast$ account of the master literature review was searched and citations relevant to each PICO question were loaded into a folder from which to conduct the evidence analysis. The evidence analysis worksheet was completed by each member of the dyad independently as a quality control measure. Using a structured PICO summary template, dyads compared their findings and produced a consensus assessment for each question and its relevant outcomes, an aggregate scoring of the strength of the evidence for that question and recommendations based upon the available evidence. Multiple outcomes could inform a recommendation, but their relative contribution to the GRADE assigned was weighed by their ranking in importance. Members were encouraged to be as explicit about judgments as possible. 


\section{Appendix C. Search Strategies for systematic review}

All searches were narrowed to English language and from 1994 -

In PubMed on December 8, 2014:

((family-centered[Text Word] OR family-centred[Text Word] OR "Family/psychology"[Mesh:noexp] OR Professional-Family[TW] OR ("PatientCentered Care"[Mesh] AND ("Family"[Mesh] OR Family[TW] OR spouse[TW] OR significant-other[TW] OR parent[TW] OR child[TW] OR children[TW] OR sibling[TW] OR friend[TW]))) AND ("Intensive Care"[Mesh] OR "Intensive Care, Neonatal"[Mesh] OR "Intensive Care Units"[Mesh] OR "Critical Care Nursing"[Mesh] OR "Critical Care"[Mesh] OR critical care[TW] OR intensive care[TW] OR Burn Unit[TW] OR Coronary Care[TW] OR Respiratory Care[TW] OR "ICU"[TW] OR "PICU"[TW] OR "NICU"[TW] NOT "Recovery Room"[Mesh])) AND English[lang]

In CINAHL on December 16, 2014

( MH "Critical Care Family Needs Inventory" OR IN "Critical Care Family Needs Inventory" ) OR ( (family-centered OR family-centred OR developmental care OR "family presence" OR (Family AND Communication) OR MH "Visitors to Patients" OR MH "Family/PF" OR MH "ProfessionalFamily Relations" OR MH "Family Attitudes" ) AND ("intensive care" OR "critical care") ) NOT PT Editorial 
In EMBASE on December 18, 2014

See next 2 pages with history printout

EMBASE search

\begin{tabular}{|c|c|c|}
\hline 1 & family centered care/ & 954 \\
\hline 2 & family attitude/ & 1903 \\
\hline 3 & exp family attitude/ & 7888 \\
\hline 4 & family coping & 600 \\
\hline 5 & $\begin{array}{l}\text { Health visitor.mp. or health } \\
\text { visitor/ }\end{array}$ & 2010 \\
\hline 6 & $\begin{array}{l}\text { social worker.mp. or social } \\
\text { worker/ }\end{array}$ & 7102 \\
\hline 7 & $\begin{array}{l}\text { Decision Support system.mp. } \\
\text { or decision support system/ }\end{array}$ & 14614 \\
\hline 8 & exp family/ & 339299 \\
\hline 9 & satisfaction/ & 29918 \\
\hline 10 & Presence.mp. & 1394139 \\
\hline 11 & $\begin{array}{l}\text { Decision making.mp. or } \\
\text { decision making/ }\end{array}$ & 269888 \\
\hline 12 & $\begin{array}{l}\text { interpersonal } \\
\text { communication.mp. or } \\
\text { interpersonal } \\
\text { communication/ }\end{array}$ & 119807 \\
\hline 13 & $\begin{array}{l}\text { Medical decision making.mp. } \\
\text { or medical decision making/ }\end{array}$ & 70263 \\
\hline 14 & $\begin{array}{l}\text { Patient care.mp. or patient } \\
\text { care/ }\end{array}$ & 252668 \\
\hline 15 & $\begin{array}{l}\text { Nurse attitude.mp. or nurse } \\
\text { attitude/ }\end{array}$ & 33623 \\
\hline 16 & $\begin{array}{l}\text { Physician attitude.mp. or } \\
\text { physician attitude/ }\end{array}$ & 42352 \\
\hline 17 & $\begin{array}{l}\text { Consultation.mp. or } \\
\text { consultation/ }\end{array}$ & 93158 \\
\hline 18 & Noise.mp. & 109246 \\
\hline 19 & $\begin{array}{l}\text { resuscitation/ or } \\
\text { resuscitation.mp. }\end{array}$ & 91232 \\
\hline 20 & Developmental care.mp. & 347 \\
\hline 21 & Family-cent?red.mp. & 3489 \\
\hline 22 & $\begin{array}{l}\text { critical care family needs } \\
\text { inventory.mp. or Critical Care } \\
\text { Family Needs Inventory/ }\end{array}$ & 78 \\
\hline 23 & $\begin{array}{l}1 \text { or } 3 \text { or } 4 \text { or } 5 \text { or } 6 \text { or } 7 \text { or } \\
22\end{array}$ & 32888 \\
\hline 24 & $\begin{array}{l}9 \text { or } 10 \text { or } 11 \text { or } 12 \text { or } 13 \text { or } \\
14 \text { or } 15 \text { or } 16 \text { or } 17 \text { or } 18\end{array}$ & 2231469 \\
\hline
\end{tabular}




\begin{tabular}{|c|c|c|}
\hline 25 & 8 AND 24 & 54583 \\
\hline 26 & 23 or 25 & 85714 \\
\hline 27 & intensive care.mp. & 218161 \\
\hline 28 & $\begin{array}{l}\text { Pediatric advanced life } \\
\text { support.mp. or pediatric } \\
\text { advanced life support/ }\end{array}$ & 580 \\
\hline 29 & critical care.mp. & 32442 \\
\hline 30 & $\begin{array}{l}\text { Resuscitation/ or } \\
\text { resuscitation.mp. }\end{array}$ & 91232 \\
\hline 31 & $\begin{array}{l}\text { (NICU or PICU or ICU).mp. } \\
\text { [mp=title, abstract, subject } \\
\text { headings, heading word, } \\
\text { drug trade name, original } \\
\text { title, device manufacturer, } \\
\text { drug manufacturer, device } \\
\text { trade name, keyword] }\end{array}$ & 71081 \\
\hline 32 & 27 or 28 or 29 or 30 or 31 & 318081 \\
\hline 33 & 26 and 32 & \\
\hline 34 & $\begin{array}{l}1 \text { or } 3 \text { or } 4 \text { or } 5 \text { or } 6 \text { or } 7 \text { or } \\
20 \text { or } 21 \text { or } 22\end{array}$ & 35621 \\
\hline 35 & 25 or 34 & 87804 \\
\hline 36 & 32 and 35 & 5282 \\
\hline 37 & (32 and 35) not Editorial.pt. & 5053 \\
\hline 38 & (32 and 35) not Editorial.pt. & 5053 \\
\hline 39 & $\begin{array}{l}\text { limit } 38 \text { to (english language } \\
\text { and } y r=* 1994-2015^{*} \text { ) }\end{array}$ & 4313 \\
\hline
\end{tabular}


Appendix D: Recommendations for future research [Print in Text Box]

\section{Family Presence in the ICU (see also Family Communication)}

- Measure long-term effects of witnessed resuscitation.

- Measure outcomes in support personnel caring for family members who choose to witness resuscitation.

- Measure outcomes related to date-night or respite.

\section{Family Support}

- Measure the value of peer-to-peer support in all age groups to improve family-centered outcomes.

- Measure the relative benefits of different approaches to providing written materials to family members.

- Further assess and measure the effect of a diary on family outcomes.

- Measure the best method of implementing a patient/family diary program.

- Assess outcomes of a decision aid on patients' surrogate decision makers' wellbeing.

\section{Communication with Family Members}

- Determine if and how communication training programs can be implemented to improve family-centered outcomes. Specifically, we recommend the following for future research: 1) validation of a quality of communication measurement tool for consistency of reported outcomes; 2) cautious use of self-assessment tools as these appear to be imprecise measures of true quality of communication; 3 ) increase the use of family-reported outcomes as the standard for quality of family communication; 4) collect data on cost of implementing communication training programs; 5 ) more long-term follow up studies since skills may dissipate over time; 6) comparison of different communication teaching modalities such as didactic training vs role-plays vs simulation; and 7) further investigation of possible risks to patients such as increased depression.

- Determine the best ways to implement open flexible visitation and fully understand the impact on family member outcomes and conflict in the ICU.

- Determine the best method of including family members in medical rounds and the impact of this on family outcomes.

- Measure the benefits and limitations of family participation in care for the adult patient population.

- Measure the impact of educational/training programs on family member outcomes and ICU LOS and costs. In addition much of the work to date has been done in pediatric populations and research should be extended to adolescents and adults of all ages.

\section{Use of Specific Consultations and ICU Team Members}

- Measure the impact of ethics consultation on family satisfaction and other familyreported outcomes.

- Measure the effectiveness and cost-effective components of ethics consultations and identify the patients for whom ethics consultation is most beneficial.

- Measure the impact of psychological support in adult and pediatric populations, with larger studies in the pre-term neonatal population needed.

- Measure the effective and cost-effective components of a palliative care consultation and identify the patients for whom palliative care consultation is most beneficial.

- Measure the impact of ethics consultation on family satisfaction and other familyreported outcomes. Further research is needed to better understand the effectiveness 
and cost-effective components of ethics consultations and to identify the patients for whom ethics consultation is most beneficial.

- Measure outcomes and return on investment from family support coordinators.

- Measure outcomes and return on investment of a patient/family navigator on family psychological symptoms, conflict and resource utilization. Determine the best navigator model, which families to target, and evaluation of any negative outcomes associated with navigators interacting with families.

- Measure the impact of a spiritual care provider on patient and family outcomes.

- Test the best method for provision of spiritual support.

- Measure outcomes from social worker intervention.

\section{Operational and Environmental Issues}

- Identify the important components of decision aids for improving outcomes.

- Measure the effect of protocols for withdrawing life support on patient- and familycentered outcomes.

- Measure the impact of executive walk rounds on family-reported outcomes.

- Measure family outcomes associated with consistency of staffing.

- Measure family outcomes associated with family inclusion in decision-making.

- Measure outcomes associated with nursing involvement in ICU decision making and the impact on family outcomes.

- Measure the effect of interventions to reduce noise or improve environmental hygiene in the ICU on outcomes important to families.

- Measure the impact of private rooms on patient and family outcomes.

- Test outcomes associated with architectural design including dedicated family space.

- Test outcomes associated with family sleep, sleep space, and napping in the ICU. 
Appendix E: Worktools

ACCM Family-Centered Care Guidelines Supplement: Work Tools for Guideline Implementation All links last accessed on 07.15.2016 
The following collaborators participated in this guidelines update, specifically with regards to creating this electronic supplement containing tools for guideline implementation.

\section{David Y. Hwang, MD (Task Force Leader)}

Assistant Professor of Neurology

Division of Neurocritical Care and Emergency Neurology

Yale School of Medicine

New Haven, CT

david.hwang@yale.edu

Judy E. Davidson, DNP RN FCCM FAAN (Guidelines Writing Group Liaison) EBP/Research Nurse Liaison

UC San Diego Health

La Jolla, CA

\section{Sarah Kraus, MPH (SCCM Staff)}

SCCM Quality and Guidelines Specialist

Mount Prospect, IL

\section{Heather M. Bullard, PharmD BCCCP}

Clinical Pharmacy Specialist, Cardiothoracic Surgery

Department of Pharmacy

The University of Chicago Medicine

Chicago, IL

\section{LeeAnn Christie, MSN RN}

Critical Care Research Scientist

Dell Children's Medical Center of Central Texas

Austin, TX

\section{Linda S. Franck, RN PhD FRCPCH FAAN}

Jack and Elaine Koehn Endowed Chair in Pediatric Nursing

Professor, Department of Family Health Care Nursing

Co-PI, UCSF Preterm Birth Initiative

University of California

San Francisco, CA

\section{Meg Frizzola, DO}

Interim Chief, Division of Critical Care Medicine

Medical Director, Pediatric Intensive Care Unit

Assistant Professor of Pediatrics

Sidney Kimmel Medical College, Thomas Jefferson University

Philadelphia, PA

\section{Serena A. Harris, PharmD BCPS BCCCP}

Clinical Pharmacy Specialist, Trauma and Surgical Critical Care

Department of Pharmacy

Eskenazi Health

Indianapolis, IN

\section{Ramona O. Hopkins, PhD}

Director, Neuroscience Center

Professor, Psychology and Neuroscience

Brigham Young University,

Provo, Utah

\section{Matthew E. Lissauer, MD}

Associate Professor of Surgery

Medical Director, Surgical Critical Care

Program Director, Surgical Critical Care Fellowship

Rutgers-Robert Wood Johnson Medical School

New Brunswick, NJ

\section{Elizabeth Scruth PhD MPH RN CCNS CCRN FCCM}

Clinical Practice Consultant, Quality and Regulatory Services

Kaiser Permanente

Oakland, CA 


\section{Category 1: Family Presence}

- Given the value family members place on presence, dissatisfaction associated with restricted presence and benefit of engagement associated with presence, that family members of critically ill patients be offered open flexible visiting hours that meets their needs while providing staff support and positive reinforcement to work in partnership with families.

\begin{tabular}{|l|l|l|l|}
\hline $\begin{array}{l}\text { American Association of Critical } \\
\text { Care Nurses (AACN) Practice Alert }\end{array}$ & $\begin{array}{l}\text { Delineates expected practice of appropriate policies to facilitate family } \\
\text { visitation, supporting evidence for open visitation in the ICU, and literature } \\
\text { available outside of AACN supporting open visitation and family presence. }\end{array}$ & Free & $\begin{array}{l}\text { http://www.aacn.org/wd/practice/content/p } \\
\text { racticealerts/family-visitation-icu-practice- } \\
\text { alert.pcms?menu=practice }\end{array}$ \\
\hline $\begin{array}{l}\text { Better Together: Partnering with } \\
\text { Families }\end{array}$ & $\begin{array}{l}\text { This North American campaign, launched by the Institute for Patient-and- } \\
\text { Family-Centered Care and supported by a coalition of Canadian } \\
\text { organizations, encourages hospitals to adopt family presence policies. } \\
\text { Website includes "change package" of free resources to facilitate 24/7 } \\
\text { family presence. }\end{array}$ & $\begin{array}{l}\text { Free } \\
\text { fogether/resources }\end{array}$ & \begin{tabular}{l} 
togs.ch/WhatWeDo/better- \\
\hline
\end{tabular} \\
\hline
\end{tabular}

- Family members of critically ill patients be offered the option of participating in interdisciplinary team rounds to improve satisfaction with communication and family engagement.

- Family members of critically ill patients be offered the option of being present during resuscitation efforts, with a staff member assigned to support the family.

\begin{tabular}{|l|l|l|l|}
\hline $\begin{array}{l}\text { American Association of Critical } \\
\text { Care Nurses Practice Alert and } \\
\text { Tools }\end{array}$ & $\begin{array}{l}\text { Provides review of current evidence supporting family presence during } \\
\text { resuscitation. Provides basic framework for nursing staff to initiate action } \\
\text { plan aimed at including families in resuscitative efforts. }\end{array}$ & Free & $\begin{array}{l}\text { http://www.aacn.org/wd/practice/content/fa } \\
\text { mily-presence-practice- } \\
\text { alert.pcms?menu=practice }\end{array}$ \\
\hline
\end{tabular}

\section{Category 2: Family Support}

- Family members of critically ill patients be offered the option to be taught how to assist with the care of their loved ones.

Apps for Communication with Mechanically Ventilated Patients

SCCM Patient Communicator App $\quad$ Contains a scale that allows patients to identify where on the body they for iPad

feel sensations of pain and itching to express the intensity of these sensations. More than 30 phrases in 19 languages that allow patients to express various needs related to their care and wellbeing.

\begin{tabular}{l|l}
$\$ 12.99$ & $\frac{\text { http://www.sccm.org/Education- }}{\text { Center/Clinical-Resources/Pages/Patient-and- }}$ \\
\hline Family.aspx
\end{tabular}




\begin{tabular}{|c|c|c|c|}
\hline $\begin{array}{l}\text { SmallTalk Intensive Care for } \\
\text { iPhone and iPad; Lingraphicare, } \\
\text { Inc }\end{array}$ & $\begin{array}{l}\text { Provides a picture-based vocabulary of phrases that patients can use to } \\
\text { communicate their needs and feelings, such as "I have chest pain" and "I } \\
\text { want a doctor." Designed for anyone who has difficulty speaking or is } \\
\text { unable to speak, due to having a language impairment, not being a native } \\
\text { speaker, being intubated, or having a tracheotomy tube. }\end{array}$ & Free & Available on iTunes \\
\hline $\begin{array}{l}\text { YoDoc for iPhone and iPad; } \\
\text { Docapps LLC }\end{array}$ & $\begin{array}{l}\text { English and Spanish language app for people on ventilators and/or people } \\
\text { with speech impairment due to critical medical condition. Patients can } \\
\text { write phrases to be spoken out, draw with their finger to show pain areas } \\
\text { and speak out pain level and intensity. }\end{array}$ & Free & Available on iTunes \\
\hline \multicolumn{4}{|c|}{ Instructional Booklet on Infant Comfort } \\
\hline $\begin{array}{l}\text { Comforting Your Baby in Intensive } \\
\text { Care }\end{array}$ & $\begin{array}{l}\text { Booklet written for parents to help them learn about infant pain and the } \\
\text { role parents can play to keep their child comfortable in the NICU, in } \\
\text { partnership with the health care team. }\end{array}$ & Free & $\begin{array}{l}\text { http://familynursing.ucsf.edu/resources- } \\
\text { parents }\end{array}$ \\
\hline
\end{tabular}

- Family education programs be included as part of clinical care as these programs have demonstrated beneficial effects for family members in the ICU.

\begin{tabular}{|c|c|c|c|}
\hline COPE for Hope & $\begin{array}{l}\text { Organization that provides on-site training for neonatal ICU staff on how to } \\
\text { provide support for NICU parents. Teaches parents how to interact with } \\
\text { their preterm infant both in the NICU and after discharge in ways that } \\
\text { enhances infant growth and development. Helps parents understand the } \\
\text { workings of the NICU unit and encourages their active engagement with } \\
\text { the NICU staff. }\end{array}$ & $\begin{array}{l}\text { Cost } \\
\text { available } \\
\text { upon } \\
\text { request }\end{array}$ & http://www.copeforhope.com/index.php \\
\hline Facilitated Sensemaking & $\begin{array}{l}\text { Article that describes a theoretical framework for providing one-on-one } \\
\text { personalized instruction to families of patients regarding the ICU } \\
\text { environment, interactions with staff, hospital services, participation in } \\
\text { patient care, and self-reflection. A list of suggested items for a "family } \\
\text { visiting kit" to supplement the instruction is provided as well. }\end{array}$ & $\begin{array}{l}\text { Free with } \\
\text { journal } \\
\text { access }\end{array}$ & $\begin{array}{l}\text { http://www.ncbi.nlm.nih.gov/pubmed/2 } \\
\underline{0234207}\end{array}$ \\
\hline
\end{tabular}

- Peer-to-peer support be implemented to improve family satisfaction, parental stress, and depression in neonatal ICUs. It is not known whether peer-to-peer support would be effective in the adult population, but work tools are provided below.

\begin{tabular}{|l|l|l|l|}
\hline Share Your Story, March of Dimes & $\begin{array}{l}\text { An online community for parents to share their experiences with } \\
\text { prematurity, birth defects, or perinatal loss }\end{array}$ & Free & http://share.marchofdimes.org/ \\
\hline SCCM THRIVE & $\begin{array}{l}\text { Contains resources to improve patient and family support after critical } \\
\text { illness, including connecting with other ICU survivors and/or family } \\
\text { members of ICU patients, finding in-person support groups, and providing } \\
\text { information on what to expect after discharge. }\end{array}$ & Free & $\underline{\text { http://www.myicucare.org/thrive/Pages/defa }}$ \\
ult.aspx \\
\hline
\end{tabular}




\begin{tabular}{|l|l|l|l|}
\hline $\begin{array}{l}\text { Intensive Care Unit Support } \\
\text { Teams for Ex-Patients (ICUsteps) }\end{array}$ & $\begin{array}{l}\text { UK resource to improve the care and support available to patients } \\
\text { recovering from critical illness and their families, including support groups. }\end{array}$ & Free & http://icusteps.org \\
\hline
\end{tabular}

- ICUs provide family with information leaflets that give information about the ICU setting.

\begin{tabular}{|l|l|l|l|}
\hline $\begin{array}{l}\text { SCCM Patient and Family Support } \\
\text { Page }\end{array}$ & $\begin{array}{l}\text { SCCM support page for ICU patients and families. Provides education on a } \\
\text { variety of ICU topics along with additional outside resource } \\
\text { recommendations. Brochures available for .pdf download. }\end{array}$ & Free & http://www.myicucare.org \\
\hline $\begin{array}{l}\text { Neurocritical Care Society Family } \\
\text { and Patient Resources }\end{array}$ & $\begin{array}{l}\text { Resource guide that provides overview of neurocritical care unit to families, } \\
\text { including members of the team, description of common diseases, and links } \\
\text { to patient and family resources. }\end{array}$ & $\begin{array}{l}\text { Free for } \\
\text { printing; } \\
\$ 40 \text { for } 25 \\
\text { brochures }\end{array}$ & $\begin{array}{l}\text { https://www.pathlms.com/ncs- } \\
\text { ondemand/categories/413/courses }\end{array}$ \\
\hline $\begin{array}{l}\text { AHRQ Guide to Patient and } \\
\text { Family Engagement in Hospital } \\
\text { Quality and Safety }\end{array}$ & $\begin{array}{l}\text { Provides written material to improve communication between patients, } \\
\text { families, and clinicians. Downloadable brochures and handouts for patients } \\
\text { and families, as well as informational poster to put in ICU. }\end{array}$ & Free & $\underline{\text { http://www.ahrq.gov }}$ \\
\hline $\begin{array}{l}\text { UCSF Critical Care Innovations } \\
\text { Group }\end{array}$ & $\begin{array}{l}\text { Extensive website designed specifically to provide information for families } \\
\text { of ICU patients, including details about ICU arrival, the ICU care team, and } \\
\text { what to expect after ICU discharge. }\end{array}$ & Free & $\underline{\text { http://ccig.ucsf.edu }}$ \\
\hline
\end{tabular}

- ICU diaries be implemented in ICUs.

\begin{tabular}{|c|c|c|c|}
\hline ICU Diary Network & $\begin{array}{l}\text { Network for healthcare providers interested in ICU diaries. Resources } \\
\text { include diary overview, literature, implementation assistance, and methods } \\
\text { for connecting with institutions who utilize an ICU diary. }\end{array}$ & Free & www.icu-diary.org \\
\hline $\begin{array}{l}\text { Josie King Foundation Care } \\
\text { Journal and App }\end{array}$ & $\begin{array}{l}\text { Journal and downloadable iPhone app to help patients and families } \\
\text { manage healthcare information. Prompts user on information to remember } \\
\text { and questions to ask healthcare team. }\end{array}$ & $\begin{array}{l}\text { App is } \\
\text { free; fee } \\
\text { for journal } \\
\text { bulk order }\end{array}$ & http://www.josieking.org \\
\hline $\begin{array}{l}\text { Graham's Foundation MyPreemie } \\
\text { App }\end{array}$ & $\begin{array}{l}\text { Downloadable iPhone, iPad, iPod, and Android application for families of } \\
\text { premature babies. Includes definitions, education, suggested questions, } \\
\text { growth charts, diary, and task list. }\end{array}$ & Free & $\begin{array}{l}\text { http://grahamsfoundation.org/resources/the } \\
\text {-mypreemie-app/ }\end{array}$ \\
\hline
\end{tabular}

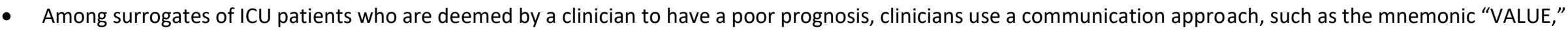
during family conferences to facilitate clinician-family communication and that validated decision support tools for family members be implemented in the ICU setting when relevant validated tools exist. 


\begin{tabular}{|c|c|c|c|}
\hline \multicolumn{4}{|l|}{ "VALUE" Mnemonic } \\
\hline "VALUE" Pocket Card & $\begin{array}{l}\text { Pocket card available for download that lists "VALUE" mnemonic for ICU } \\
\text { communication with families: value family statements, acknowledge family } \\
\text { emotions, listen to family, understand the patient as a person, elicit family } \\
\text { questions. }\end{array}$ & Free & $\begin{array}{l}\text { http://depts.washington.edu/eolcare/produc } \\
\underline{\text { ts/communication-tools/ }}\end{array}$ \\
\hline \multicolumn{4}{|l|}{ Decision Support Tools } \\
\hline Prolonged Mechanical Ventilation & $\begin{array}{l}\text { Appendix for this article contains a pilot-tested decision aid for families of } \\
\text { patients who have been mechanically ventilated for longer than } 10 \text { days } \\
\text { and who are re-evaluating patient goals-of-care. Associated with improved } \\
\text { communication, decreased cost, and positive physician reviews. Currently } \\
\text { being developed in web format. }\end{array}$ & $\begin{array}{l}\text { Free with } \\
\text { journal } \\
\text { access }\end{array}$ & $\begin{array}{l}\text { http://www.ncbi.nlm.nih.gov/pubmed/22635 } \\
\underline{048}\end{array}$ \\
\hline $\begin{array}{l}\text { Ottawa Patient Decision Aid } \\
\text { Research Group Life Support } \\
\text { Decision Aid }\end{array}$ & $\begin{array}{l}\text { Field-tested paper-based decision aid for families of ICU patients who are } \\
\text { making decisions regarding life support versus comfort care. }\end{array}$ & Free & $\begin{array}{l}\text { https://decisionaid.ohri.ca/docs/das/Critically } \\
\text { III Decision Support.pdf }\end{array}$ \\
\hline $\begin{array}{l}\text { CARENET Code Status Decision } \\
\text { Aid }\end{array}$ & $\begin{array}{l}\text { Paper-based decision aid for deciding on code status, developed by the } \\
\text { Canadian Researchers at the End of Life Network. }\end{array}$ & Free & $\begin{array}{l}\text { http://thecarenet.ca/docs/CPR\%20Decision\% } \\
\text { 20Aid\%20revised\%20to\%20PDF\%20brochure } \\
\text { \%20Nov\%203\%202009.pdf }\end{array}$ \\
\hline
\end{tabular}

\section{Category 3: Family Communication}

- Healthcare clinicians in the ICU use structured approaches to communication such as that included in the "VALUE" mnemonic when engaging in communication with family members, specifically active listening, expressions of empathy, and making supportive statements around non-abandonment and decision-making. In addition, we suggest that family members of critically ill patients be offered a written brochure.

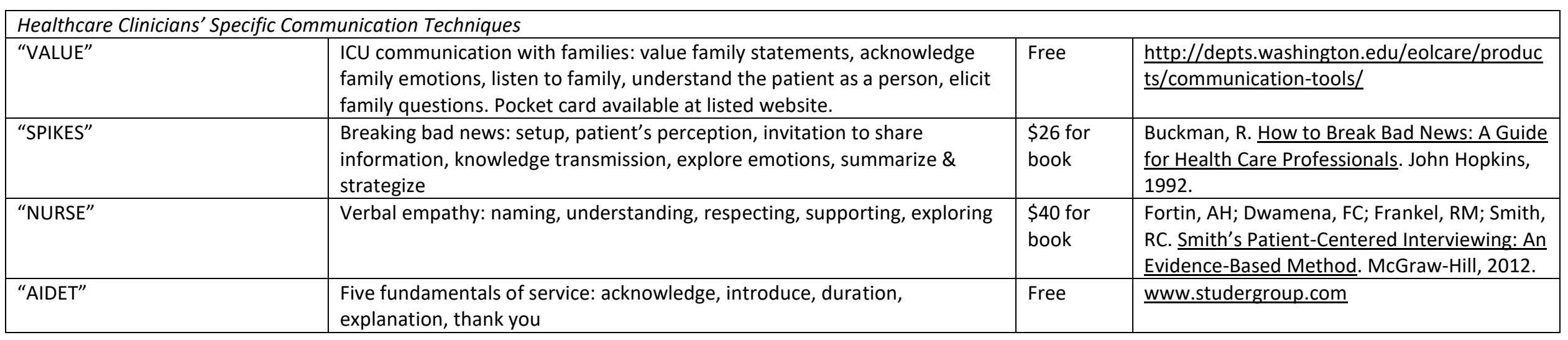




\begin{tabular}{|c|c|c|c|}
\hline \multicolumn{4}{|c|}{ Structured Approaches to Family Communication Improvement } \\
\hline $\begin{array}{l}\text { Rhode Island ICU Collaborative } \\
\text { Communication Process } \\
\text { Measures }\end{array}$ & $\begin{array}{l}\text { Description of "day } 1 \text { " and "day } 3 \text { " goals for family communication that a } \\
\text { statewide coalition of ICUs targeted for a coordinated ICU communication } \\
\text { quality improvement project. }\end{array}$ & $\begin{array}{l}\text { Free with } \\
\text { journal } \\
\text { access }\end{array}$ & $\begin{array}{l}\text { http://www.ncbi.nlm.nih.gov/pubmed/24060 } \\
\underline{769}\end{array}$ \\
\hline $\begin{array}{l}\text { Robert Wood Johnson } \\
\text { Foundation Palliative Care Quality } \\
\text { Measures }\end{array}$ & $\begin{array}{l}\text { Describes a proposed set of quality measures for ICUs to target with } \\
\text { respect to palliative care, divided by domains; the majority of the domains } \\
\text { relate to communication with and support of the family, with structured } \\
\text { goals that should be achieved during an ICU admission. }\end{array}$ & $\begin{array}{l}\text { Free with } \\
\text { journal } \\
\text { access }\end{array}$ & $\begin{array}{l}\text { http://www.ncbi.nlm.nih.gov/pubmed/17057 } \\
\text { 606 } \\
\text { http://www.ncbi.nlm.nih.gov/pubmed/14501 } \\
\underline{954}\end{array}$ \\
\hline $\begin{array}{l}\text { VHA Care and Communication } \\
\text { "Bundle" }\end{array}$ & $\begin{array}{l}\text { Describes "bundle of care" that includes identification of surrogate decision } \\
\text { makers and patient preferences, communication timelines, social work, } \\
\text { and spiritual support; with performance measurement and feedback. }\end{array}$ & $\begin{array}{l}\text { Free with } \\
\text { journal } \\
\text { access }\end{array}$ & $\begin{array}{l}\text { http://www.ncbi.nlm.nih.gov/pubmed/16885 } \\
\underline{251}\end{array}$ \\
\hline $\begin{array}{l}\text { University of Maryland Family } \\
\text { Meeting Algorithm }\end{array}$ & $\begin{array}{l}\text { Description of algorithm (e.g., introduction of clinical team, identification of } \\
\text { surrogate decision makers and advance directives, palliative care } \\
\text { involvement) designed to improve ICU communication, including time- } \\
\text { based checklist (i.e., benchmarks at } 24,72 \text {, and } 96 \text { hours), as well as } \\
\text { triggers to escalate the family communication algorithm. }\end{array}$ & $\begin{array}{l}\text { Free with } \\
\text { journal } \\
\text { access }\end{array}$ & $\begin{array}{l}\text { http://www.ncbi.nlm.nih.gov/pubmed/24085 } \\
\underline{828}\end{array}$ \\
\hline \multicolumn{4}{|l|}{ Bereavement Brochure } \\
\hline $\begin{array}{l}\text { Bereavement Support Leaflet, } \\
\text { Saint-Louis Teaching Hospital } \\
\text { (France) }\end{array}$ & $\begin{array}{l}\text { This appendix to a randomized trial examining the positive effects of a } \\
\text { proactive end-of-life conference strategy on long-term adverse } \\
\text { psychological outcomes among families contains an extensive brochure } \\
\text { that was used in the study to counsel families on bereavement. }\end{array}$ & $\begin{array}{l}\text { Free with } \\
\text { journal } \\
\text { access }\end{array}$ & $\begin{array}{l}\text { http://www.ncbi.nlm.nih.gov/pubmed/17267 } \\
\underline{907}\end{array}$ \\
\hline
\end{tabular}

- Based on existing evidence of patient and family burdens associated with poor communication, as well as improved clinician-reported skills and comfort following communication training, we suggest that ICU clinicians receive family-centered communication training as one element of an overall well-rounded critical care training curriculum and ongoing education.

\begin{tabular}{|l|l|l|l|}
\hline Communication Training Programs for Clinicians \\
\hline $\begin{array}{l}\text { AHRQ Communication Training } \\
\text { Slides }\end{array}$ & $\begin{array}{l}\text { Provides definitions of patient and family engagement and overview of } \\
\text { communication skills; includes practice exercises in the form of } \\
\text { patient/provider scenarios. }\end{array}$ & Free & http://www.ahrq.gov \\
\hline Breaking Bad News Foundation & $\begin{array}{l}\text { Communication skills training program developed by a neonatologist where } \\
\text { physicians and healthcare workers participate in improvisational role- } \\
\text { playing sessions with professional actors. Sessions are video-taped and } \\
\text { watched remotely by certified instructors. Foundation will work with } \\
\text { institutions to fit their local needs. }\end{array}$ & $\begin{array}{l}\text { Costs } \\
\text { available } \\
\text { upon } \\
\text { request }\end{array}$ & \begin{tabular}{l} 
http://www.bbnfoundation.org/ \\
\hline
\end{tabular} \\
\hline
\end{tabular}




\begin{tabular}{|c|c|c|c|}
\hline $\begin{array}{l}\text { Center to Advance Palliative Care } \\
\text { (CAPC) }\end{array}$ & $\begin{array}{l}\text { Formal membership to CAPC includes access to numerous CME/CEU } \\
\text { courses. }\end{array}$ & $\begin{array}{l}\text { Range } \\
\text { from } \$ 600 \\
\text { for critical } \\
\text { access to } \\
\$ 7500 \text { for } \\
\text { institution }\end{array}$ & https://www.capc.org/capc-central/courses/ \\
\hline DocCom & $\begin{array}{l}\text { Comprehensive online program with }>400 \text { video modules demonstrating } \\
\text { communication skills. Includes evidence-based recommendations, skills } \\
\text { checklists, and assessment questions. }\end{array}$ & $\begin{array}{l}\text { \$95/yr for } \\
\text { individual; } \\
\$ 40+\text { per } \\
\text { person } \\
\text { group } \\
\text { pricing; } \\
\$ 25+\text { per } \\
\text { person } \\
\text { library } \\
\text { pricing }\end{array}$ & $\begin{array}{l}\text { http://www.aachonline.org/dnn/DocCom.asp } \\
\underline{x}\end{array}$ \\
\hline IMPACT-ICU & $\begin{array}{l}\text { Quality improvement program at the University of California system } \\
\text { designed to integrate palliative care into the ICU by training and supporting } \\
\text { bedside nurses. Internet link is to training manual outlining the } \\
\text { implementation of communication skills training workshops for nurses. }\end{array}$ & Free & https://ucsf.box.com/ImpactICUProjectGuide \\
\hline $\begin{array}{l}\text { Institute for Healthcare } \\
\text { Communication }\end{array}$ & $\begin{array}{l}\text { Offers variety of workshops on communication, including disclosing } \\
\text { unanticipated medical outcomes, end-of-life conversations, and challenging } \\
\text { clinician-patient relationships. }\end{array}$ & $\begin{array}{l}\text { Costs } \\
\text { available } \\
\text { upon } \\
\text { request }\end{array}$ & http://healthcarecomm.org/training/ \\
\hline Vital Talk & $\begin{array}{l}\text { Online communication skills courses available for clinicians who work with } \\
\text { patients with serious illness and end-of-life care, focused on balancing } \\
\text { honesty with empathy. }\end{array}$ & $\begin{array}{l}\text { Costs } \\
\text { available } \\
\text { upon } \\
\text { request }\end{array}$ & www.vitaltalk.org \\
\hline
\end{tabular}

\section{Category 4: Consultations}

- Proactive palliative care consultation be provided to decrease ICU and hospital length of stay among selected critically ill patients. 


\begin{tabular}{|c|c|c|c|}
\hline $\begin{array}{l}\text { Center to Advance Palliative Care } \\
\text { - Improving Palliative Care in the } \\
\text { ICU Project (CAPC / IPAL) }\end{array}$ & $\begin{array}{l}\text { Free resource available on website regarding the implementation of } \\
\text { screening criteria for palliative care in ICUs. }\end{array}$ & Free & $\begin{array}{l}\text { https://media.capc.org/filer_public/80/be/80 } \\
\text { be3587-6ca1-4eb8-93f0- } \\
\text { 7fa0e30cd153/76 66 ipal-icu-implementing- } \\
\text { icu-screening-criteria-for-unmet-palliative- } \\
\text { care-needs.pdf }\end{array}$ \\
\hline $\begin{array}{l}\text { National Consensus Project for } \\
\text { Quality Palliative Care Clinical } \\
\text { Practice Guidelines }\end{array}$ & $\begin{array}{l}\text { Guidelines providing a "blueprint" upon which to build optimal palliative } \\
\text { care. The document comments on the issue of primary vs. consultative } \\
\text { palliative care. }\end{array}$ & Free & $\begin{array}{l}\text { http://www.nationalconsensusproject.org/gu } \\
\text { ideline.pdf }\end{array}$ \\
\hline
\end{tabular}

- Ethics consultation, particularly in reaction to a conflict about goals of care for an ICU patient, be provided to decrease ICU and hospital length of stay among selected critically ill patients.

Joint Professional Societies

Statement on Responding to

Requests for Potentially

Inappropriate Treatments in ICUs
Provides a protocol for early consultation with experts in communication and conflict negotiation, namely ethics or palliative care. It is recommended that these practitioners become involved early when conflict is arising, and that they facilitate frequent, effective

communication between healthcare providers and the patients and/or their surrogates.

\begin{tabular}{l|l|}
$\begin{array}{l}\text { Free with } \\
\text { journal } \\
\text { access }\end{array}$ & $\underline{\text { http://www.ncbi.nlm.nih.gov/pubmed/25978 }}$ \\
& \\
\hline
\end{tabular}

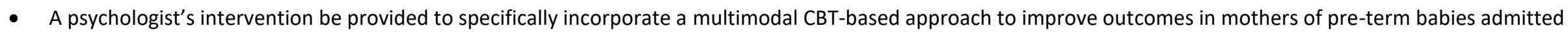
to the NICU.

\begin{tabular}{|c|c|c|c|}
\hline $\begin{array}{l}\text { Behavioral Interventions for } \\
\text { Mothers of Pre-Term Babies }\end{array}$ & $\begin{array}{l}\text { A manualized 6-session treatment based on traum-focused cognitive- } \\
\text { behavioral therapy that includes: (1) psychoeducation to educate mothers } \\
\text { about PTSD and common feelings and thoughts of NICU parents; (2) } \\
\text { cognitive restructuring to help mothers recognize and challenge erroneous } \\
\text { and maladaptive cognitions; ( } 3 \text { ) progressive muscle relaxation to reduce } \\
\text { anxiety; and (4) development and processing of the mother's trauma } \\
\text { narrative. The intervention has been shown in a single center study to be } \\
\text { effective in reducing symptoms of parental trauma, anxiety, and depression } \\
\text { in the NICU and at 6-month follow-up. }\end{array}$ & $\begin{array}{l}\text { Free with } \\
\text { journal } \\
\text { access }\end{array}$ & $\begin{array}{l}\text { http://www.ncbi.nlm.nih.gov/pubmed/23909 } \\
\underline{669}\end{array}$ \\
\hline
\end{tabular}

- Targeted video and reading materials be provided in the context of psychological support provided to mothers of pre-term babies admitted to the ICU.

- Social workers be included within an interdisciplinary team to participate in family meetings and improve family satisfaction. 
- Family navigators assigned to families throughout ICU stay be considered in the ICU to improve family satisfaction with physician communication, psychological symptoms, and reduce length of ICU or hospital stay and costs of care.

\begin{tabular}{|c|c|c|c|}
\hline $\begin{array}{l}\text { Published Training Protocol for } \\
\text { ICU "Facilitators" }\end{array}$ & $\begin{array}{l}\text { Describes a protocol for a trained nurse or social worker "communication } \\
\text { facilitator" designed to improve psychological distress among family } \\
\text { members of critically ill patients, patient length of stay in the intensive care } \\
\text { unit and hospital, and costs associated with care and the intervention. }\end{array}$ & $\begin{array}{l}\text { Free with } \\
\text { journal } \\
\text { access }\end{array}$ & $\begin{array}{l}\text { http://www.ncbi.nlm.nih.gov/pubmed/22772 } \\
\underline{089}\end{array}$ \\
\hline
\end{tabular}

- Given the consistency of expression of family values for availability of spiritual care, the accreditation standard requirements, and the results of the observation study families should be offered spiritual support with a spiritual advisor or chaplain.

\section{Category 5: Operational and Environmental Issues}

- Protocols be implemented to ensure adequate and standardized use of sedation and analgesia during withdrawal of life support.

University of Washington Withdrawal of Life Support

Orders for Adults

\begin{abstract}
Provides guidance for use of analgesics and sedatives in addition to discontinuation of other therapies not required when adult patients are transitioned to a goal of comfort care. Also describes a set of principles to assist transitioning goals-of-care to promote comfort instead of focusing on disease processes.
\end{abstract}

\begin{tabular}{|l|l|} 
Free & $\frac{\text { http://depts.washington.edu/eolcare/pubs/w }}{\text { p-content/uploads/2011/08/wls-orders2.pdf }}$ \\
& \\
\end{tabular}

- Specialized nurse be used as part of an overall program to potentially decrease ICU and hospital length of stay and to possibly improve quality of communication in the ICU. Hospitals implement policies to promote family-centered care in the ICU to improve family experience.

\begin{tabular}{|c|c|c|c|}
\hline $\begin{array}{l}\text { Institute for Patient- and Family- } \\
\text { Centered Care (work tools for } \\
\text { hospital-wide leaders) }\end{array}$ & $\begin{array}{l}\text { Contains links to multiple well-established tools for executives; including } \\
\text { "How to Get Started," which details a step-wise plan for hospitals to build } \\
\text { partnerships with patients and families; and 'Strategies for Leadership," } \\
\text { which includes a video, resource guides, and a hospital self-assessment } \\
\text { inventory. }\end{array}$ & Free & $\begin{array}{l}\text { http://www.ipfcc.org/tools/downloads- } \\
\text { tools.html }\end{array}$ \\
\hline $\begin{array}{l}\text { Institute for Patient- and Family- } \\
\text { Centered Care (work tools for ICU } \\
\text { leaders) }\end{array}$ & $\begin{array}{l}\text { Assessment tools that provide teams with a structure for assessing their } \\
\text { unit and hospital for the four principles of patient andfamily-centered care } \\
\text { in ten areas. }\end{array}$ & $\$ 10$ each & $\begin{array}{l}\text { http://www.ipfcc.org/resources/other/index. } \\
\underline{\text { html }}\end{array}$ \\
\hline
\end{tabular}

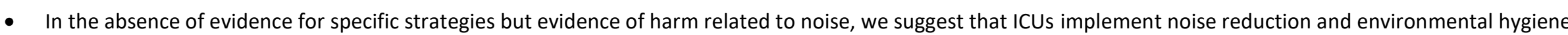
practices and the use of private rooms to improve patient/family satisfaction and noise reduction while managing staff stress imposed with the change from openbay to private rooms in the neonatal ICU. 
ACCM Guidelines for Intensive

Care Unit Design

Recommended Standards for

Newborn ICU Design
Expert opinion on optimal ICU design, including extensive recommendations for designing a "family support zone."

Expert opinion on optimal neonatal ICU design, including extensive recommendations for family support space.
Free

Free 137

https://www3.nd.edu/ nicudes/Recommend edNICUStandardsFinal8Aug15.pdf 
Electronic Supplement Table 6: Recommendations rank ordered by importance of outcome

\section{OUTCOME AND \\ AVERAGE CLINICIAN RATING \\ (10 = highest rating)}

Family Psychological Symptoms 9.62

\section{ICU FAMILY-CENTERED CARE RECOMMENDATIONS}

- A psychologist-delivered trauma-focused multimodal cognitive behavioral therapy (CBT) or targeted video and reading materials providing psychological support be provided to mothers of pre-term babies admitted to the Neonatal Intensive Care Unit (NICU). (Rec 1.3)

- Family navigators be assigned to families throughout the ICU stay. (Rec 1.5)

- Healthcare clinicians in the ICU use structured approaches to communication with family members, and family members of critically ill patients undergoing withdrawal of life support be offered a written bereavement brochure. (Rec 2.2)

- Family members of critically ill patients be offered the option of being present during resuscitation efforts, with a staff member assigned to support the family. (Rec 3.3)

- Family members of critically ill children be offered the option to be taught how to assist with the care of their critically ill child. (Rec 4.1)

- Family education programs be included as part of clinical care. (Rec 4.2)

- Peer-to-peer support be implemented in pediatric ICUs. (Rec 4.3)

- ICUs provide family with leaflets that give information about the ICU setting. (Rec 4.4)

- ICU diaries be implemented in ICUs. (Rec 4.5)

Quality of Communication

7.67
- ICU clinicians receive family-centered communication training as one element of a critical care training program. (Rec 2.3)

- Validated decision support tools for family members be implemented in the ICU setting when relevant validated tools exist. (Rec 4.6)

- Among surrogates of ICU patients who are deemed by a clinician to have a poor prognosis, clinicians use a communication approach during family conferences and validated decision support tools for family members be implemented in the ICU setting. (Rec 4.7)

- Nurses be trained to provide support for family members. (Rec 5.2) 
Family Trust in Clinicians

7.66

Family Satisfaction with Care 7.33

- Routine interdisciplinary family conferences be used in the ICU. (Rec 2.1)

- Medical social workers be included within an interdisciplinary team to participate in family meetings. (Rec 1.4$)$

- Family members of critically ill patients be offered open flexible visiting that meets their needs while providing support for staff and positive reinforcement to work in partnership with families. (Rec 3.1)

- Family education programs be included as part of clinical care. (Rec 4.2)

- Peer-to-peer support be implemented in pediatric ICUs. (Rec 4.3)

- Hospitals implement policies to promote family-centered care in the ICU. (Rec 5.3)

- ICUs implement noise reduction and environmental hygiene practices and use private rooms. (Rec 5.4)

- Family sleep be considered and families are provided a sleep surface to reduce the effects of sleep deprivation. (Rec 5.7)

Family Satisfaction with Communication

7.33

- Spiritual support from a spiritual advisor or chaplain be offered to families of ICU patients to meet their expressed desire for spiritual care. (Rec 1.1)

- Family navigators be assigned to families throughout the ICU stay. (Rec 1.5)

- Routine interdisciplinary family conferences be used in the ICU. (Rec 2.1)

- Healthcare clinicians in the ICU use structured approaches to communication with family members, and family members of critically ill patients undergoing withdrawal of life support be offered a written bereavement brochure. (Rec 2.2)

- ICU clinicians receive family-centered communication training as one element of a critical care training program. (Rec 2.3)

- Family members of critically ill patients be offered the option of participating in interdisciplinary team rounds. (Rec 3.2)

- Validated decision support tools for family members be implemented in the ICU setting when relevant validated tools exist. (Rec 4.6)

Family Self-Efficacy 6.93
- Family members of critically ill children be offered the option to be taught how to assist with the care of their critically ill child. (Rec 4.1)

Family or Clinician Conflict

- Routine interdisciplinary family conferences be used in the ICU. (Rec 2.1) 
- Validated decision support tools for family members be implemented in the ICU setting when relevant validated tools exist. (Rec 4.6)

Clinician Rated Quality of Dying 6.30 - Protocols be implemented to ensure adequate and standardized use of sedation and analgesia during withdrawal
of life support. ( $\operatorname{Rec} 5.1)$

6.22

ICU and Hospital Utilization

- Proactive palliative care consultation be provided among selected critically ill patients. (Rec 1.1)

- Ethics consultation be provided among critically ill patients for whom there is a conflict about goals of care. (Rec 1.2)

- Family navigators be assigned to families throughout the ICU stay. (Rec 1.5)

- Routine interdisciplinary family conferences be used in the ICU. (Rec 2.1)

- Nurses be trained to provide support for family members. (Rec 5.2)

Clinician Self-Efficacy 5.59
- ICU clinicians receive family-centered communication training as one element of a critical care training program. (Rec 2.3) 
Figure 1. Citation analysis of family-centered care publication from Thomson Web of Science

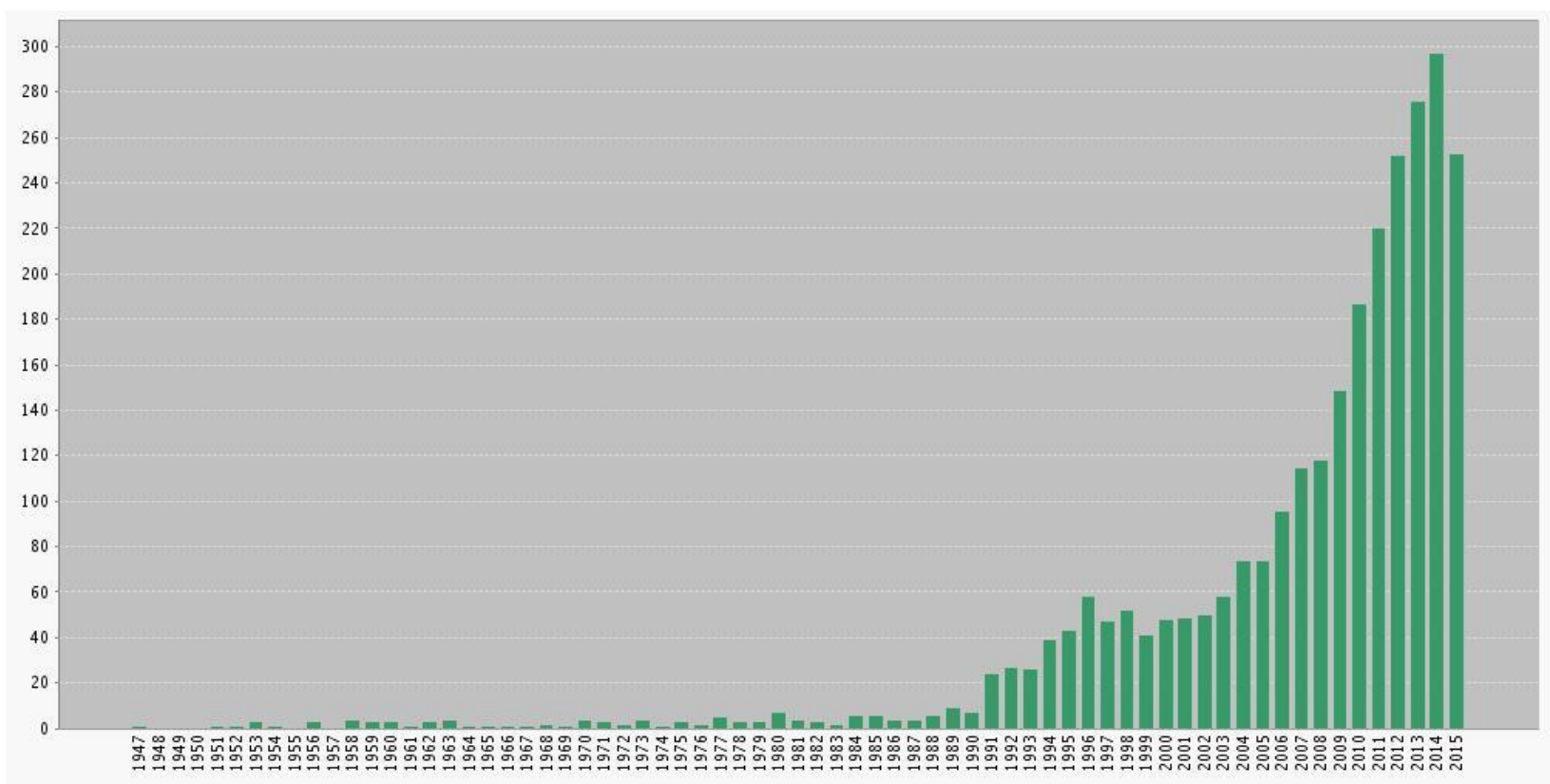


Figure 2: Prisma Flow Diagram for Systematic Review

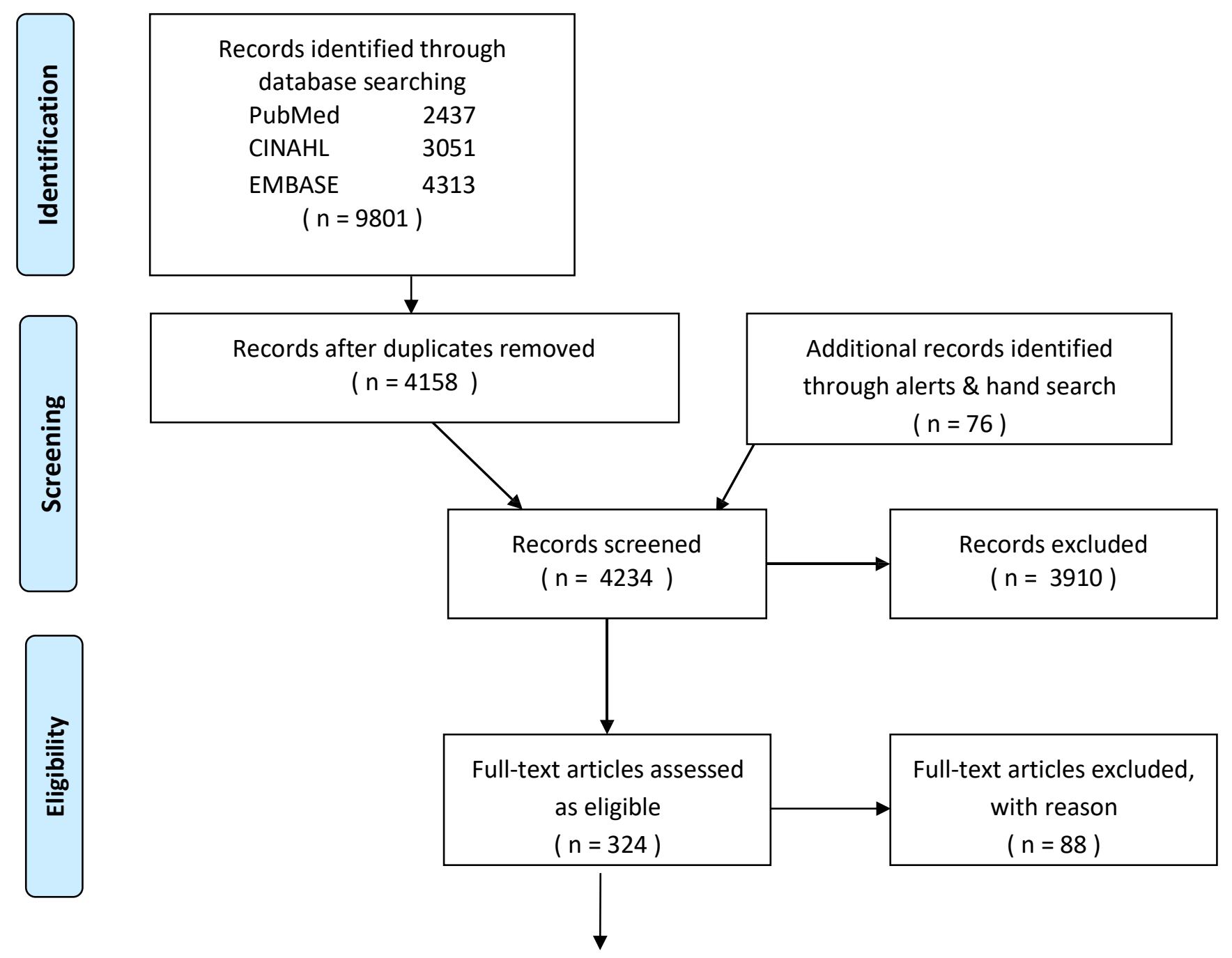




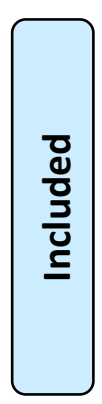

Studies included

$(n=236)$

Adapted from: Moher D, Liberati A, Tetzlaff J, Altman DG, The PRISMA Group (2009). Preferred Reporting Items for Systematic Reviews and Meta- Analyses: The PRISMA Statement. PLoS Med 6(6): e1000097. doi:10.1371/journal.pmed1000097 


\section{References}

1. Prendergast TJ: Resolving conflicts surrounding end-of-life care. New Horiz 1997;5:62-71

2. Desai SV, Law TJ, Needham DM: Long-term complications of critical care. Crit Care Med 2011;39:371-379

3. Needham DM, Davidson J, Cohen H, et al: Improving long-term outcomes after discharge from intensive care unit: Report from a stakeholders' conference. Crit Care Med 2012;40:502-509

4. Lautrette A, Darmon M, Megarbane B, et al: A communication strategy and brochure for relatives of patients dying in the ICU. $N$ Engl J Med 2007;356:469-478

5. Davidson JE, Jones C, Bienvenu OJ: Family response to critical illness: Postintensive care syndrome-family. Crit Care Med 2012;40:618-624

6. Adelman RD, Tmanova LL, Delgado D, et al: Caregiver burden: A clinical review. JAMA 2014;311:1052-1060

7. Lynn J: Strategies to ease the burden of family caregivers. JAMA 2014;311:1021-1022

8. Davidson JE, Powers K, Hedayat KM, et al: Clinical practice guidelines for support of the family in the patient-centered intensive care unit: American college of critical care medicine task force 2004-2005. Crit Care Med 2007;35:605-622 
9. Council of Medical Specialty Societies: Principles for the development of specialty society clinical guidelines. Chicago, CMSS, 2012

10. McMaster University: Health information research unit (HIRU) search filters for MEDLINE.

http://hiru.mcmaster.ca/hiru/HIRU Hedges MEDLINE Strategies.aspx\#Qualitative Edition. 2003

11. Wilczynski NL, Marks S, Haynes RB: Search strategies for identifying qualitative studies in CINAHL. Qual Health Res $2007 ; 17: 705-710$

12. McAlvin SS, Carew-Lyons A: Family presence during resuscitation and invasive procedures in pediatric critical care: A systematic review. Am J Crit Care 2014;23:477-485

13. Staniszewska S, Brett J, Redshaw M, et al: The POPPY study: Developing a model of family-centred care for neonatal units. Worldviews on Evidence-Based Nursing 2012;9:243-255

14. Visser M, Deliens L, Houttekier D: Physician-related barriers to communication and patient and family-centred decision making towards the end of life in intensive care: A systematic review. Crit Care 2014;18:604

15. Ciufo D, Hader R, Holly C: A comprehensive systematic review of visitation models in adult critical care units within the context of patient- and family-centred care. Int J Evid Based Healthc 2011;9:362-387 
16. Boudreaux ED, Francis JL, Loyacano T: Family presence during invasive procedures and resuscitations in the emergency department: A critical review and suggestions for future research. Ann Emerg Med 2002;40:193-205

17. Power N, Franck L: Parent participation in the care of hospitalized children: A systematic review. J Adv Nurs 2008;62:622-641

18. Clarke E, Curtis J, Luce J, et al: Quality indicators for end-of-life care in the intensive care unit. Crit Care Med 2003;31:2255-2262

19. Cleveland LM: Parenting in the neonatal intensive care unit. J Obstet Gynecol Neonatal Nurs 2008;37:666-691

20. Halm MA: Family presence during resuscitation: A critical review of the literature. Am J Crit Care 2005;14:494-511

21. Shields L, Zhou H, Pratt J, et al: Family-centred care for hospitalised children aged 0-12 years. Cochrane Database of Systematic Reviews 2012;DOI: 10.1002/14651858.CD004811.pub3.

22. Cypress BS: Family presence on rounds: A systematic review of literature. Dimens Crit Care Nurs 2012;31:53-64

23. Fawole OA, Dy SM, Wilson RF, et al: A systematic review of communication quality improvement interventions for patients with advanced and serious illness. J Gen Intern Med 2013;28:570-577

24. Aslakson R, Cheng J, Vollenweider D, et al: Evidence-based palliative care in the intensive care unit: A systematic review of interventions. Journal of Palliative Medicine 2014;17:219-235 
25. Guyatt GH, Oxman AD, Kunz R, et al: Going from evidence to recommendations. BMJ 2008;336:1049-1051

26. Al-Mutair A, Plummer V, Clerehan R, et al: Needs and experiences of intensive care patients' families: A saudi qualitative study. Nurs Crit Care 2014;19:135-144

27. Boyles C, Langley R: The transfer of parents from picu to the ward. Pediatric Critical Care Medicine 2011;1):A64-A65

28. Jaeschke R, Guyatt GH, Dellinger P, et al: Use of GRADE grid to reach decisions on clinical practice guidelines when consensus is elusive. BMJ 2008;337:a744

29. Schunemann HJ, Jaeschke R, Cook DJ, et al: An official ATS statement: Grading the quality of evidence and strength of recommendations in ATS guidelines and recommendations. Am J Respir Crit Care Med 2006;174:605-614

30. Kynoch K, Chang A, Coyer F, et al: The effectiveness of interventions to meet family needs of critically ill patients in an adult intensive care unit: A systematic review protocol update. JBI Database of Systematic Reviews and Implementation Reports $2014 ; 12: 14-26$

31. Karlsson C, Tisell A, Engstrom A, et al: Family members' satisfaction with critical care: A pilot study. Nurs Crit Care 2011;16:1118 
32. Macdonald ME, Liben S, Carnevale FA, et al: An office or a bedroom? challenges for family-centered care in the pediatric intensive care unit. J Child Health Care 2012;16:237-249

33. Soury-Lavergne A, Hauchard I, Dray S, et al: Survey of caregiver opinions on the practicalities of family-centred care in intensive care units. J Clin Nurs 2012;21:1060-1067

34. Raz S, Goren Y, Artzman P, et al: Initial experience with a family support group in the ICU. Intensive Care Medicine 2010;36:S156

35. Plakas S, Taket A, Cant B, et al: The meaning and importance of vigilant attendance for the relatives of intensive care unit patients. Nurs Crit Care 2014;19:243-254

36. Burr G: Contextualizing critical care family needs through triangulation: An australian study. Intensive Crit Care Nurs $1998 ; 14: 161-169$

37. Davidson JE, Daly BJ, Agan D, et al: Facilitated sensemaking: Testing of a mid-range theory of family support. Commun Nurs Res 2009;42:353-353

38. Olsen KD, Dysvik E, Hansen BS: The meaning of family members' presence during intensive care stay: A qualitative study. Intensive Crit Care Nurs 2009;25:190-198 
39. Giannini A, Miccinesi G, Prandi E, et al: Partial liberalization of visiting policies and ICU staff: A before-and-after study. Intensive Care Med 2013;39:2180-2187

40. Melnyk BM, Alpert-Gillis L, Feinstein NF, et al: Creating opportunities for parent empowerment: Program effects on the mental health/coping outcomes of critically ill young children and their mothers. Pediatrics 2004;113:e597-607

41. Melnyk BM, Crean HF, Feinstein NF, et al: Testing the theoretical framework of the COPE program for mothers of critically ill children: An integrative model of young children's post-hospital adjustment behaviors. Journal of Pediatric Psychology 2007;32:463474

42. Melnyk BM, Feinstein NF, Alpert-Gillis L, et al: Reducing premature infants' length of stay and improving parents' mental health outcomes with the creating opportunities for parent empowerment (COPE) neonatal intensive care unit program: A randomized, controlled trial. Pediatrics 2006;118:e1414-27

43. Baharoon S, Al Yafi W, Al Qurashi A, et al: Family satisfaction in critical care units: Does an open visiting hours policy have an impact? J Patient Saf 2014;

44. Henneman EA, McKenzie JB, Dewa CS: An evaluation of interventions for meeting the information needs of families of critically ill patients. Am J Crit Care 1992;1:85-93 
45. Chapman DK, Collingridge DS, Mitchell LA, et al: Satisfaction with elimination of all visitation restrictions in a mixed-profile intensive care unit. Am J Crit Care 2016;25:46-50

46. Mangram AJ, Mccauley T, Villarreal D, et al: Families' perception of the value of timed daily "family rounds" in a trauma ICU. Am Surg 2005;71:886-891

47. Schiller WR, Anderson BF: Family as a member of the trauma rounds: A strategy for maximized communication. J Trauma Nurs 2003;10:93-101

48. Tripathi S, Arteaga G, Rohlik G, et al: Implementation of patient-centered bedside rounds in the pediatric intensive care unit. $J$ Nurs Care Qual 2015;30:160-166

49. Stickney CA, Ziniel SI, Brett MS, et al: Family participation during intensive care unit rounds: Attitudes and experiences of parents and healthcare providers in a tertiary pediatric intensive care unit. J Pediatr 2014;164:402-6.e1-4

50. Drago MJ, Aronson PL, Madrigal V, et al: Are family characteristics associated with attendance at family centered rounds in the PICU? Pediatr Crit Care Med 2013;14:e93-7

51. Bramwell R, Weindling M: Families' views on ward rounds in neonatal units. Archives of Disease in Childhood: Fetal and Neonatal Edition 2005;90:F429-F431 
52. Grzyb MJ, Coo H, Ruhland L, et al: Views of parents and health-care providers regarding parental presence at bedside rounds in a neonatal intensive care unit. J Perinatol 2014;34:143-148

53. Ladak LA, Premji SS, Amanullah MM, et al: Family-centered rounds in pakistani pediatric intensive care settings: Nonrandomized pre- and post-study design. Int J Nurs Stud 2013;50:717-726

54. Voos KC, Ross G, Ward MJ, et al: Effects of implementing family-centered rounds (FCRs) in a neonatal intensive care unit (NICU). J Matern Fetal Neonatal Med 2011;24:1403-1406

55. Jacobowski NL, Girard TD, Mulder JA, et al: Families in critical care. communication in critical care: Family rounds in the intensive care unit. Am J Crit Care 2010;19:421-430

56. Cameron MA, Schleien CL, Morris MC: Parental presence on pediatric intensive care unit rounds. J Pediatr 2009;155:522-528

57. Aronson PL, Yau J, Helfaer MA, et al: Impact of family presence during pediatric intensive care unit rounds on the family and medical team. Pediatrics 2009;124:1119-1125

58. Egging D, Crowley M, Arruda T, et al: Emergency nursing resource: Family presence during invasive procedures and resuscitation in the emergency department. J Emerg Nurs 2011;37:469-473 
59. ECC Committee, Subcommittees and Task Forces of the American Heart Association: 2005 american heart association guidelines for cardiopulmonary resuscitation and emergency cardiovascular care. Circulation 2005;112:IV1-203

60. Family presence during CPR and invasive procedures. AACN NEWS 2004;21:4-4

61. American Academy of Pediatrics Committee on Pediatric Emergency Medicine, American College of Emergency Physicians Pediatric Emergency Medicine Committee, O'Malley P, et al: Patient- and family-centered care and the role of the emergency physician providing care to a child in the emergency department. Pediatrics 2006;118:2242-2244

62. Fulbrook P, Latour J, Albarran J, et al: The presence of family members during cardiopulmonary resuscitation: European federation of critical care nursing associations, european society of paediatric and neonatal intensive care and european society of cardiology council on cardiovascular nursing and allied professions joint position statement. Eur J Cardiovasc Nurs 2007;6:255-258

63. Moons P, Norekval TM: European nursing organizations stand up for family presence during cardiopulmonary resuscitation: A joint position statement. Progress in cardiovascular nursing 2008;23:136-139

64. Meyers TA, Eichhorn DJ, Guzzetta CE: Do families want to be present during CPR? A retrospective survey. Journal of emergency nursing: JEN : official publication of the Emergency Department Nurses Association 1998;24:400-405

65. Mazer MA, Cox LA, Capon JA: The public's attitude and perception concerning witnessed cardiopulmonary resuscitation. Critical Care Medicine 2006;34:2925-2928 
66. Duran CR, Oman KS, Abel JJ, et al: Attitudes toward and beliefs about family presence: A survey of healthcare providers, patients' families, and patients. American Journal of Critical Care 2007;16:270-280

67. Ong MEH, Chung WL, Mei JSE: Comparing attitudes of the public and medical staff towards witnessed resuscitation in an asian population. Resuscitation 2007;73:103-108

68. Tinsley C, Hill JB, Shah J, et al: Experience of families during cardiopulmonary resuscitation in a pediatric intensive care unit. Pediatrics 2008;122:e799-804

69. Zakaria M, Siddique M: Presence of family members during cardio-pulmonary resuscitation after necessary amendments. Journal of the Pakistan Medical Association 2008;58:632-635

70. Ersoy G, Yanturali S, Suner S, et al: Turkish patient relatives' attitudes towards family-witnessed resuscitation and affecting sociodemographic factors. European Journal of Emergency Medicine 2009;16:188-193

71. Mortelmans LJM, Van Broeckhoven V, Van Boxstael S, et al: Patients'and relatives'view on witnessed resuscitation in the emergency department: A prospective study. European Journal of Emergency Medicine 2010;17:203-207

72. Hung MSY, Pang SMC: Family presence preference when patients are receiving resuscitation in an accident and emergency department. J Adv Nurs 2011;67:56-67 
73. Leung NY, Chow SK: Attitudes of healthcare staff and patients' family members towards family presence during resuscitation in adult critical care units. J Clin Nurs 2012;21:2083-2093

74. Chew KS, Ghani ZA: Attitudes and perceptions of the general malaysian public regarding family presence during resuscitation. Singapore Medical Journal 2014;55:439-442

75. Maxton FJ: Parental presence during resuscitation in the PICU: The parents' experience. sharing and surviving the resuscitation: A phenomenological study. J Clin Nurs 2008;17:3168-3176

76. Leske JS, McAndrew NS, Brasel KJ: Experiences of families when present during resuscitation in the emergency department after trauma. J Trauma Nurs 2013;20:77-85

77. MacLean SL, Guzzetta CE, White C, et al: Family presence during cardiopulmonary resuscitation and invasive procedures: Practices of critical care and emergency nurses. Am J Crit Care 2003;12:246-257

78. Fallis WM, McClement S, Pereira A: Family presence during resuscitation: A survey of canadian critical care nurses' practices and perceptions. Dynamics 2008;19:22-28

79. Goldberger ZD, Nallamothu BK, Nichol G, et al: Policies allowing family presence during resuscitation and patterns of care during in-hospital cardiac arrest. Circ Cardiovasc Qual Outcomes 2015;8:226-234 
80. Meyers TA, Eichhorn DJ, Guzzetta CE, et al: Family presence during invasive procedures and resuscitation. The American journal of nursing 2000;100:32-42; quiz 43

81. Redley B, Hood K: Staff attitudes towards family presence during resuscitation. Accident and emergency nursing 1996;4:145-151

82. McClenathan BM, Torrington KG, Uyehara CF: Family member presence during cardiopulmonary resuscitation: A survey of US and international critical care professionals. Chest 2002;122:2204-2211

83. Wacht O, Dopelt K, Snir Y, et al: Attitudes of emergency department staff toward family presence during resuscitation. Israel Medical Association Journal 2010;12:366-370

84. Al Mutair A, Plummer V, Paul O'Brien A, et al: Attitudes of healthcare providers towards family involvement and presence in adult critical care units in saudi arabia: A quantitative study. J Clin Nurs 2014;23:744-755

85. Sacchetti A, Carraccio C, Leva E, et al: Acceptance of family member presence during pediatric resuscitations in the emergency department: Effects of personal experience. Pediatric Emergency Care 2000;16:85-87

86. Feagan LM, Fisher NJ: The impact of education on provider attitudes toward family-witnessed resuscitation. J Emerg Nurs $2011 ; 37: 231-239$ 
87. Jarvis AS: Parental presence during resuscitation: Attitudes of staff on a paediatric intensive care unit. Intensive Crit Care Nurs $1998 ; 14: 3-7$

88. Grice AS, Picton P, Deakin CD: Study examining attitudes of staff, patients and relatives to witnessed resuscitation in adult intensive care units. Br J Anaesth 2003;91:820-824

89. Ong MEH, Chan YH, Srither DE, et al: Asian medical staff attitudes towards witnessed resuscitation. Resuscitation 2004;60:4550

90. Knott A, Kee CC: Nurses' beliefs about family presence during resuscitation. Applied Nursing Research 2005;18:192-198

91. Yanturali S, Ersoy G, Yuruktumen A, et al: A national survey of turkish emergency physicians perspectives regarding family witnessed cardiopulmonary resuscitation. International Journal of Clinical Practice 2005;59:441-446

92. Macy C, Lampe E, O'Neil B, et al: The relationship between the hospital setting and perceptions of family-witnessed resuscitation in the emergency department. Resuscitation 2006;70:74-79

93. Badir A, Sepit D: Family presence during CPR: A study of the experiences and opinions of turkish critical care nurses. Int $J$ Nurs Stud 2007;44:83-92 
94. Kirchhoff C, Stegmaier J, Buhmann S, et al: Trauma surgeons' attitude towards family presence during trauma resuscitation: A nationwide survey. Resuscitation 2007;75:267-275

95. Lam DSY, Wong SN, Hui H, et al: Attitudes of doctors and nurses to family presence during paediatric cardiopulmonary resuscitation. Hong Kong Journal of Paediatrics 2007;12:253-259

96. Gunes UY, Zaybak A: A study of turkish critical care nurses' perspectives regarding family-witnessed resuscitation. J Clin Nurs 2009;18:2907-2915

97. Koberich S, Kaltwasser A, Rothaug O, et al: Family witnessed resuscitation - experience and attitudes of german intensive care nurses. Nurs Crit Care 2010;15:241-250

98. Kianmehr N, Mofidi M, Rahmani H, et al: The attitudes of team members towards family presence during hospital-based CPR: A study based in the muslim setting of four iranian teaching hospitals. Journal of the Royal College of Physicians of Edinburgh $2010 ; 40: 4-8$

99. Gordon ED, Kramer E, Couper I, et al: Family-witnessed resuscitation in emergency departments: Doctors' attitudes and practices. South African Medical Journal 2011;101:765-767

100. Kosowan S, Jensen L: Family presence during cardiopulmonary resuscitation: Cardiac health care professionals' perspectives. Canadian journal of cardiovascular nursing = Journal canadien en soins infirmiers cardio-vasculaires 2011;21:23-29 
101. Al-Mutair AS, Plummer V, Copnell B: Family presence during resuscitation: A descriptive study of nurses' attitudes from two saudi hospitals. Nurs Crit Care 2012;17:90-98

102. Mahabir D, Sammy I: Attitudes of ED staff to the presence of family during cardiopulmonary resuscitation: A trinidad and tobago perspective. Emergency Medicine Journal 2012;29:817-820

103. Tripon C, Defossez G, Ragot S, et al: Parental presence during cardiopulmonary resuscitation of children: The experience, opinions and moral positions of emergency teams in france. Archives of Disease in Childhood 2014;99:310-315

104. Salamonson Y, van Heere B, Everett B, et al: Voices from the floor: Nurses' perceptions of the medical emergency team. Intensive Crit Care Nurs 2006;22:138-143

105. Kingsnorth J, O'Connell K, Guzzetta CE, et al: Family presence during trauma activations and medical resuscitations in a pediatric emergency department: An evidence-based practice project. Journal of Emergency Nursing 2010;36:115-121

106. Robinson SM, Mackenzie-Ross S, Campbell Hewson GL, et al: Psychological effect of witnessed resuscitation on bereaved relatives. Lancet 1998;352:614-17

107. Holzhauser K, Finucane J, De Vries SM: Family presence during resuscitation: A randomised controlled trial of the impact of family presence. Australasian Emergency Nursing Journal 2006;8:139-147 
108. Jabre P, Belpomme V, Azoulay E, et al: Family presence during cardiopulmonary resuscitation. New England Journal of Medicine 2013;368:1008-1018

109. Jabre P, Tazarourte K, Azoulay E, et al: Offering the opportunity for family to be present during cardiopulmonary resuscitation: 1-year assessment. Intensive Care Medicine 2014;40:981-987

110. Pasquale MA, Pasquale MD, Baga $L$, et al: Family presence during trauma resuscitation: Ready for primetime? $J$ Trauma 2010;69:1092-9; discussion 1099-100

111. Ebrahim S, Singh S, Parshuram CS: Parental satisfaction, involvement, and presence after pediatric intensive care unit admission. J Crit Care 2013;28:40-45

112. Oman KS, Duran CR: Health care providers' evaluations of family presence during resuscitation. Journal of Emergency Nursing 2010;36:524-533

113. Mian P, Warchal S, Whitney S, et al: Impact of a multifaceted intervention on nurses' and physicians' attitudes and behaviors toward family presence during resuscitation. Critical Care Nurse 2007;27:52-61

114. Mangurten J, Scott SH, Guzzetta CE, et al: Effects of family presence during resuscitation and invasive procedures in a pediatric emergency department. Journal of Emergency Nursing 2006;32:225-233 
115. Franck LS, Oulton K, Nderitu S, et al: Parent involvement in pain management for NICU infants: A randomized controlled trial. Pediatrics 2011;128:510-518

116. Bastani F, Abadi TA, Haghani $\mathrm{H}$ : Effect of family-centered care on improving parental satisfaction and reducing readmission among premature infants: A randomized controlled trial. J Clin Diagn Res 2015;9:SC04-8

117. van der Pal SM, Maguire CM, le Cessie S, et al: Parental experiences during the first period at the neonatal unit after two developmental care interventions. Acta Paediatr 2007;96:1611-1616

118. Azoulay E, Pochard F: Communication with family members of patients dying in the intensive care unit. Curr Opin Crit Care $2003 ; 9: 545-550$

119. Cooper LG, Gooding JS, Gallagher J, et al: Impact of a family-centered care initiative on NICU care, staff and families. J Perinatol 2007;27 Suppl 2:S32-7

120. Chien W, Chiu YL, Lam L, et al: Effects of a needs-based education programme for family carers with a relative in an intensive care unit: A quasi-experimental study. Int J Nurs Stud 2006;43:39-50

121. Chiu YL, Chien WT, Lam LW: Effectiveness of a needs-based education programme for families with a critically ill relative in an intensive care unit. J Clin Nurs 2004;13:655-656 
122. Oswalt KL, McClain DB, Melnyk B: Reducing anxiety among children born preterm and their young mothers. MCN Am J Matern Child Nurs 2013;38:144-149

123. Melnyk BM, Crean HF, Feinstein NF, et al: Maternal anxiety and depression after a premature infant's discharge from the neonatal intensive care unit: Explanatory effects of the creating opportunities for parent empowerment program. Nurs Res $2008 ; 57: 383-394$

124. Ahn YM, Kim NH: Parental perception of neonates, parental stress and education for NICU parents. Asian Nurs Res (Korean Soc Nurs Sci) $2007 ; 1: 199-210$

125. Chien W, Ip W, Lee I: Psychometric properties of a chinese version of the critical care family needs inventory. Res Nurs Health $2005 ; 28: 474-487$

126. McQueen D, Lakes K, Rich J, et al: Feasibility of a caregiver-assisted exercise program for preterm infants. J PERINAT NEONAT NURS 2013;27:184-192

127. Pearson J, Andersen K: Evaluation of a program to promote positive parenting in the neonatal intensive care unit. NEONAT NETW $2001 ; 20: 43-48$

128. Tearl DK, Hertzog JH: Home discharge of technology-dependent children: Evaluation of a respiratory-therapist driven family education program. Respiratory Care 2007;52:171-176 
129. Huning BM, Reimann M, Beerenberg U, et al: Establishment of a family-centred care programme with follow-up home visits: Implications for clinical care and economic characteristics. Klin Padiatr 2012;224:431-436

130. Melnyk BM, Feinstein NF: Reducing hospital expenditures with the COPE (creating opportunities for parent empowerment) program for parents and premature infants: An analysis of direct healthcare neonatal intensive care unit costs and savings. Nurs Adm Q 2009;33:32-37

131. Rossman B, Engstrom JL, Meier PP, et al: "They've walked in my shoes": Mothers of very low birth weight infants and their experiences with breastfeeding peer counselors in the neonatal intensive care unit. J Hum Lact 2011;27:14-24

132. Rossman B, Engstrom JL, Meier PP: Healthcare providers' perceptions of breastfeeding peer counselors in the neonatal intensive care unit. Res Nurs Health 2012;35:460-474

133. Rossman B, Greene MM, Meier PP: The role of peer support in the development of maternal identity for "NICU moms". J Obstet Gynecol Neonatal Nurs 2015;44:3-16

134. Hurst I: One size does not fit all: Parents' evaluations of a support program in a newborn intensive care nursery. $J$ Perinat Neonatal Nurs 2006;20:252-261

135. Preyde M, Ardal F: Effectiveness of a parent "buddy" program for mothers of very preterm infants in a neonatal intensive care unit. CMAJ : Canadian Medical Association journal = journal de l'Association medicale canadienne 2003;168:969-973 
136. Pochard F, Darmon M, Fassier T, et al: Symptoms of anxiety and depression in family members of intensive care unit patients before discharge or death. A prospective multicenter study. J Crit Care 2005;20:90-96

137. Azoulay E, Pochard F, Kentish-Barnes N, et al: Risk of post-traumatic stress symptoms in family members of intensive care unit patients. Am J Respir Crit Care Med 2005;171:987-994

138. Kentish-Barnes N, Lemiale V, Chaize M, et al: Assessing burden in families of critical care patients. Crit Care Med 2009;37:S448-56

139. Azoulay E, Pochard F, Chevret S, et al: Impact of a family information leaflet on effectiveness of information provided to family members of intensive care unit patients: A multicenter, prospective, randomized, controlled trial. Am J Respir Crit Care Med $2002 ; 165: 438-442$

140. Azoulay E, Chevret S, Leleu G, et al: Half the families of intensive care unit patients experience inadequate communication with physicians. Crit Care Med 2000;28:3044-3049

141. Peigne V, Chaize M, Falissard B, et al: Important questions asked by family members of intensive care unit patients. Crit Care Med $2011 ; 39: 1365-1371$

142. Jones C, Backman C, Griffiths RD: Intensive care diaries and relatives' symptoms of posttraumatic stress disorder after critical illness: A pilot study. Am J Crit Care 2012;21:172-176 
143. Garrouste-Orgeas M, Coquet I, Perier A, et al: Impact of an intensive care unit diary on psychological distress in patients and relatives* ${ }^{*}$ Crit Care Med 2012;40:2033-2040

144. Garrouste-Orgeas M, Perier A, Mouricou P, et al: Writing in and reading ICU diaries: Qualitative study of families' experience in the ICU. PLoS One 2014;9:e110146

145. Bergbom I, Svensson C, Berggren E, et al: Patients' and relatives' opinions and feelings about diaries kept by nurses in an intensive care unit: Pilot study. Intensive Crit Care Nurs 1999;15:185-191

146. Backman CG, Walther SM: Use of a personal diary written on the ICU during critical illness. Intensive Care Med 2001;27:426429

147. Cox CE, Lewis CL, Hanson LC, et al: Development and pilot testing of a decision aid for surrogates of patients with prolonged mechanical ventilation. Critical Care Medicine 2012;40:2327-2334

148. Kodali S, Stametz R, Clarke D, et al: Implementing family communication pathway in neurosurgical patients in an intensive care unit. Palliat Support Care 2014;1-7

149. Holzapfel L, Demingeon G, Piralla B, Biot L, Nallet B: A four-step protocol for limitation of treatment in terminal care. an observatinal study in 475 intensive care unit pateints. Intensive Care Med2002;28:1309-1315 
150. Hwang DY, Yagoda D, Perrey $\mathrm{H}$, et al: Assessment of satisfaction with care among family members of survivors in a neuroscience intensive care unit. Neurocritical Care 2012;17:S261

151. Selph RB, Shiang J, Engelberg R, et al: Empathy and life support decisions in intensive care units. J Gen Intern Med 2008;23:1311-1317

152. Stapleton RD, Engelberg RA, Wenrich MD, et al: Clinician statements and family satisfaction with family conferences in the intensive care unit. Crit Care Med 2006;34:1679-1685

153. McDonagh JR, Elliott TB, Engelberg RA, et al: Family satisfaction with family conferences about end-of-life care in the intensive care unit: Increased proportion of family speech is associated with increased satisfaction. Crit Care Med 2004;32:1484-1488

154. White DB, Braddock $\mathrm{CH}, 3$ rd, Bereknyei S, et al: Toward shared decision making at the end of life in intensive care units: Opportunities for improvement. Arch Intern Med 2007;167:461-467

155. Pham K, Thornton JD, Engelberg RA, et al: Alterations during medical interpretation of ICU family conferences that interfere with or enhance communication. Chest 2008;134:109-116

156. Van Cleave A,C., Roosen-Runge M, Miller A, B., et al: Quality of communication in interpreted versus noninterpreted PICU family meetings*. Crit Care Med 2014;42:1507-1517 
157. Thornton JD, Pham K, Engelberg RA, et al: Families with limited english proficiency receive less information and support in interpreted intensive care unit family conferences. Crit Care Med 2009;37:89-95

158. Lilly CM, De Meo DL, Sonna LA, et al: An intensive communication intervention for the critically ill. American Journal of Medicine 2000;109:469-475

159. Daly BJ, Douglas SL, O'Toole E, et al: Effectiveness trial of an intensive communication structure for families of long-stay ICU patients. Chest 2010;138:1340-1348

160. Lilly CM, Sonna LA, Haley KJ, et al: Intensive communication: Four-year follow-up from a clinical practice study. Crit Care Med 2003;31:S394-9

161. Michelson KN, Clayman ML, Haber-Barker N, et al: The use of family conferences in the pediatric intensive care unit. J Palliat Med 2013;16:1595-1601

162. Wall RJ, Curtis JR, Cooke CR, et al: Family satisfaction in the ICU: Differences between families of survivors and nonsurvivors. Chest 2007;132:1425-1433

163. Abbott KH, Sago JG, Breen CM, et al: Families looking back: One year after discussion of withdrawal or withholding of lifesustaining support. Crit Care Med 2001;29:197-201 
164. Hunziker S, McHugh W, Sarnoff-Lee B, et al: Predictors and correlates of dissatisfaction with intensive care. Crit Care Med $2012 ; 40: 1554-1561$

165. Quenot JP, Rigaud JP, Chahraoui K, et al: Is the level of satisfaction of families of patients in critical care improved by the quality of the information they receive? Intensive Care Medicine 2010;36:S323

166. Belayachi J, Himmich S, Madani N, et al: Psychological burden in inpatient relatives: The forgotten side of medical management. QJM 2014;107:115-122

167. Hartog CS, Schwarzkopf D, Riedemann NC, et al: End-of-life care in the intensive care unit: A patient-based questionnaire of intensive care unit staff perception and relatives' psychological response. Palliat Med 2015;29:336-345

168. Baile WF, Buckman R, Lenzi R, et al: SPIKES-A six-step protocol for delivering bad news: Application to the patient with cancer. Oncologist 2000;5:302-311

169. Shaw D, J., Davidson J, E., Smilde R, I., et al: Multidisciplinary team training to enhance family communication in the ICU*. Crit Care Med 2014;42:265-271

170. Fenwick J, Barclay L, Schmied V: Interactions in neonatal nurseries: Women's perceptions of nurses and nursing. J NEONAT NURS 2000;6:197-203 
171. Shudy M, de Almeida ML, Ly S, et al: Impact of pediatric critical illness and injury on families: A systematic literature review. Pediatrics 2006;118 Suppl 3:S203-18

172. Kentish-Barnes N, Chaize M, Seegers V, et al: Complicated grief after death of a relative in the intensive care unit. Eur Respir $J$ $2015 ; 45: 1341-1352$

173. McCallister JW, Gustin JL, Wells-Di Gregorio S, et al: Communication skills training curriculum for pulmonary and critical care fellows. Ann Am Thorac Soc 2015;12:520-525

174. Krimshtein NS, Luhrs CA, Puntillo KA, et al: Training nurses for interdisciplinary communication with families in the intensive care unit: An intervention. J Palliat Med 2011;14:1325-1332

175. Boss RD, Urban A, Barnett MD, et al: Neonatal critical care communication (NC3): Training NICU physicians and nurse practitioners. J Perinatol 2013;33:642-646

176. Downar J, Knickle K, Granton JT, et al: Using standardized family members to teach communication skills and ethical principles to critical care trainees. Crit Care Med 2012;40:1814-1819

177. Hales BM, Hawryluck L: An interactive educational workshop to improve end of life communication skills. J Contin Educ Health Prof 2008;28:241-8 
178. Hope AA, Hsieh SJ, Howes JM, et al: Let's talk critical: Development and evaluation of a communication skills training program for critical care fellows. Ann Am Thorac Soc 2015;12:505-511

179. Meyer EC, Sellers DE, Browning DM, et al: Difficult conversations: Improving communication skills and relational abilities in health care. PEDIATR CRIT CARE MED 2009;10:352-359

180. Meyer EC, Brodsky D, Hansen AR, et al: An interdisciplinary, family-focused approach to relational learning in neonatal intensive care. Journal of Perinatology 2011;31:212-219

181. Pye S, Kane J, Jones A: Parental presence during pediatric resuscitation: The use of simulation training for cardiac intensive care nurses. J SPEC PEDIATR NURS 2010;15:172-175

182. Bays AM, Engelberg RA, Back AL, et al: Interprofessional communication skills training for serious illness: Evaluation of a smallgroup, simulated patient intervention. J Palliat Med 2014;17:159-166

183. Garcia de Lucio L, Garcia Lopez FJ, Marin Lopez MT, et al: Training programme in techniques of self-control and communication skills to improve nurses' relationships with relatives of seriously ill patients: A randomized controlled study. $J$ Adv Nurs 2000;32:425-431

184. Morton J, Blok GA, Reid C, et al: The european donor hospital education programme (EDHEP): Enhancing communication skills with bereaved relatives. Anaesth Intensive Care 2000;28:184-190 
185. Curtis JR, Back AL, Ford DW, et al: Effect of communication skills training for residents and nurse practitioners on quality of communication with patients with serious illness: A randomized trial. JAMA 2013;310:2271-2281

186. Campbell ML, Guzman JA: Impact of a proactive approach to improve end-of-life care in a medical ICU. Chest 2003;123:266271

187. Campbell ML, Guzman JA: A proactive approach to improve end-of-life care in a medical intensive care unit for patients with terminal dementia. Crit Care Med 2004;32:1839-1843

188. Norton SA, Hogan LA, Holloway RG, et al: Proactive palliative care in the medical intensive care unit: Effects on length of stay for selected high-risk patients. Crit Care Med 2007;35:1530-1535

189. Lamba S, Murphy P, McVicker S, et al: Changing end-of-life care practice for liver transplant service patients: Structured palliative care intervention in the surgical intensive care unit. J Pain Symptom Manage 2012;44:508-519

190. Mosenthal AC, Murphy PA, Barker LK, et al: Changing the culture around end-of-life care in the trauma intensive care unit. $J$ Trauma 2008;64:1587-1593

191. Khandelwal N, Kross EK, Engelberg RA, et al: Estimating the effect of palliative care interventions and advance care planning on ICU utilization: A systematic review. Crit Care Med 2015;43:1102-1111 
192. Andereck WS, McGaughey JW, Schneiderman LJ, et al: Seeking to reduce nonbeneficial treatment in the ICU: An exploratory trial of proactive ethics intervention. Critical Care Medicine 2014;42:824-830

193. Schneiderman LJ, Gilmer T, Teetzel HD: Impact of ethics consultations in the intensive care setting: A randomized, controlled trial. Crit Care Med 2000;28:3920-3924

194. Schneiderman LJ, Gilmer T, Teetzel HD, et al: Effect of ethics consultations on nonbeneficial life-sustaining treatments in the intensive care setting: A randomized controlled trial. JAMA 2003;290:1166-1172

195. Dowdy MD, Robertson C, Bander JA: A study of proactive ethics consultation for critically and terminally ill patients with extended lengths of stay. Crit Care Med 1998;26:252-259

196. Alam J, Ahlund S, Thalange N, et al: Psychological support for parents in UK tertiary-level neonatal units - A postcode lottery. Archives of Disease in Childhood 2010;95:A104

197. Tunick RA, Gavin JA, DeMaso DR, et al: Pediatric psychology critical care consultation: An emerging subspecialty. Clinical Practice in Pediatric Psychology 2013;1:42-54

198. Conniff H, Pierce C, Brierley J, et al: Communication on picu: Reflections on consultant conversations with families. Pediatric Critical Care Medicine 2011;1):A71 
199. Carvalho AE, Linhares MB, Padovani FH, et al: Anxiety and depression in mothers of preterm infants and psychological intervention during hospitalization in neonatal ICU. Span J Psychol 2009;12:161-170

200. Shaw RJ, St John N, Lilo EA, et al: Prevention of traumatic stress in mothers with preterm infants: A randomized controlled trial. Pediatrics 2013;132:e886-94

201. Shaw RJ, St John N, Lilo E, et al: Prevention of traumatic stress in mothers of preterms: 6-month outcomes. Pediatrics $2014 ; 134: e 481-8$

202. Peris A, Bonizzoli M, lozzelli D, et al: Early intra-intensive care unit psychological intervention promotes recovery from post traumatic stress disorders, anxiety and depression symptoms in critically ill patients. Crit Care 2011;15:R41

203. Burns JP, Mello MM, Studdert DM, et al: Results of a clinical trial on care improvement for the critically ill. Crit Care Med $2003 ; 31: 2107-2117$

204. Sundararajan K, Sullivan TR, Chapman M: Determinants of family satisfaction in the intensive care unit. Anaesth Intensive Care $2012 ; 40: 159-165$

205. Curtis JR, Treece PD, Nielsen EL, et al: Randomized trial of communication facilitators to reduce family distress and intensity of end-of-life care. Am J Respir Crit Care Med 2015;193:154-162 
206. Chaboyer W, Thalib L, Alcorn K, et al: The effect of an ICU liaison nurse on patients and family's anxiety prior to transfer to the ward: An intervention study. Intensive Crit Care Nurs 2007;23:362-369

207. Shelton W, Moore CD, Socaris S, et al: The effect of a family support intervention on family satisfaction, length-of-stay, and cost of care in the intensive care unit. Crit Care Med 2010;38:1315-1320

208. Moore CD, Bernardini GL, Hinerman R, et al: The effect of a family support intervention on physician, nurse, and family perceptions of care in the surgical, neurological, and medical intensive care units. Crit Care Nurs Q 2012;35:378-387

209. Al-Mutair A, Plummer V, Clerehan R, et al: Families' needs of critical care muslim patients in saudi arabia: A quantitative study. Nurs Crit Care 2014;19:185-195

210. Puggina A, da Silva M, de Araújo M: Messages from relatives of patients in coma: Hope as common element. Acta Paul Enfermagem 2008;21:249-255

211. Anderson W, Cimino J, Ungar A, et al: Keys to communicating about prognosis in the ICU: A multicenter study of family, provider, and expert perspectives (FR424-C). Journal of Pain and Symptom Management 2013;45 (2):382

212. Gries CJ, Engelberg RA, Kross EK, et al: Predictors of symptoms of posttraumatic stress and depression in family members after patient death in the ICU. Chest 2010;137:280-287 
213. Robinson MR, Thiel MM, Backus MM, et al: Matters of spirituality at the end of life in the pediatric intensive care unit. Pediatrics 2006;118:e719-e729

214. The Joint Commission: Specifications manual for joint commission national quality core measures. 2015A1 Edition. Oak Brook, IL, Joint Commission Resources, 2015

215. Johnson JR, Engelberg RA, Nielsen EL, et al: The association of spiritual care providers' activities with family members' satisfaction with care after a death in the ICU*. Crit Care Med 2014;42:1991-2000

216. Halm MA, Myers RN, Bennetts P: Providing spiritual care to cardiac patients: Assessment and implications for practice. Crit Care Nurse 2000;20:54

217. Wall RJ, Engelberg RA, Gries CJ, et al: Spiritual care of families in the intensive care unit. Crit Care Med 2007;35:1084-1090

218. Campbell ML, Field BE: Management of the patient with do not resuscitate status: Compassion and cost containment. Heart Lung 1991;20:345-348

219. Sedillot N, Holzapfel L, Jacquet-Francillon T, et al: A five-step protocol for withholding and withdrawing of life support in an emergency department: An observational study. European Journal of Emergency Medicine 2008;15:145-149 
220. Sjokvist $P$, Sundin $P$, Berggren L: Limiting life support

experiences with a special protocol. Acta Anaesthesiologica Scandinavica 1998;42:232-237

221. Treece PD, Engelberg RA, Crowley L, et al: Evaluation of a standardized order form for the withdrawal of life support in the intensive care unit. Crit Care Med 2004;32:1141-1148

222. Licata J, Aneja R, K., Kyper C, et al: In our unit. A foundation for patient safety: Phase I implementation of interdisciplinary bedside rounds in the pediatric intensive care unit. Crit Care Nurse 2013;33:89-91

223. Penticuff $\mathrm{JH}$, Arheart KL: Effectiveness of an intervention to improve parent-professional collaboration in neonatal intensive care. J Perinat Neonatal Nurs 2005;19:187-202

224. Paganini MC, Bousso RS: Nurses' autonomy in end-of-life situations in intensive care units. Nurs Ethics 2015;22:803-814

225. Ahrens T, Yancey V, Kollef M: Improving family communications at the end of life: Implications for length of stay in the intensive care unit and resource use. Am J Crit Care 2003;12:317-23; discussion 324

226. White DB, Cua SM, Walk R, et al: Nurse-led intervention to improve surrogate decision making for patients with advanced critical illness. Am J Crit Care 2012;21:396-409 
227. Nelson DP, Polst G: An interdisciplinary team approach to evidence-based improvement in family-centered care. Crit Care Nurs Q 2008;31:110-118

228. Perrault C, Coates A, Collinge J, et al: Family support system in newborn medicine: Does it work? follow-up study of infants at risk. J Pediatr 1986;108:1025-1030

229. Kinsala E: Focus on. the VERY IMPORTANT PARTNER program: Integrating family and friends into the health care experience. Prog Cardiovasc Nurs 1999;14:103-110

230. Zeschke J: Diminishingtheexperienceof anxietyforparents of children admitted into the pediatric intensive care unit. Pediatric Critical Care Medicine 2011;1):A166-A167

231. Trochelman K, Albert N, Spence J, et al: Patients and their families weigh in on evidence-based hospital design. Crit Care Nurse 2012;32:e1-e10

232. Shahheidari M, Homer C: Impact of the design of neonatal intensive care units on neonates, staff, and families: A systematic literature review. J Perinat Neonatal Nurs 2012;26:260-6; quiz 267-8

233. Meaburn A, Boylan A, Ford D, et al: Improving patient care during transitions from the ICU to the general care floor via staff and family collaboration and a novel patient/familycentered transfer brochure. Critical Care Medicine. Conference: 42nd Critical Care Congress of the Society of Critical Care Medicine, SCCM 2013;40: 
234. Heinemann A, Hellstrom-Westas L, Nyqvist KH: Factors affecting parents' presence with their extremely preterm infants in a neonatal intensive care room. Acta Paediatrica 2013;102:695-702

235. Riera MA, Gallart E, Afonso E, et al: Stressful perceptions of tracheostomized patients in the intensive care unit. Intensive Care Medicine. Conference: 27th Annual Congress of the European Society of Intensive Care Medicine, ESICM 2014;40:S275-S275

236. Byers JF, Yovaish W, Lowman LB, et al: Co-bedding versus single-bedding premature multiple-gestation infants in incubators. JOGNN 2003;32:340-347

237. Domanico R, Davis DK, Coleman F, et al: Documenting the NICU design dilemma: Parent and staff perceptions of open ward versus single family room units. Journal of Perinatology 2010;30:343-351

238. Day A, Haj-Bakri S, Lubchansky S, et al: Sleep, anxiety and fatigue in family members of patients admitted to the intensive care unit: A questionnaire study. Critical Care 2013;17:1-7

239. Verceles A, Corwin D, Afshar M, et al: Half of the family members of critically ill patients experience excessive daytime sleepiness.. Intensive Care Med 2014;40:1124

240. Beck SA, Weis J, Greisen G, et al: Room for family-centered care - a qualitative evaluation of a neonatal intensive care unit remodeling project. Journal of Neonatal Nursing 2009;15:88-99 
241. Watson J, DeLand M, Gibbins S, et al: Improvements in staff quality of work life and family satisfaction following the move to single-family room NICU design. Adv Neonatal Care 2014;14:129-136

242. Thompson DR, Hamilton DK, Cadenhead CD, et al: Guidelines for intensive care unit design. Crit Care Med 2012;40:1586-1600

243. Singer S, Tucker A: The evolving literature on safety WalkRounds: Emerging themes and practical messages. BMJ QUAL SAF $2014 ; 789$

244. Thomas E, Sexton J, Neilands T, et al: The effect of executive walk rounds on nurse safety climate attitudes: A randomized trial of clinical units. BMC Health Services Research 2005;5:28-

245. Saladino L, Pickett L, Frush K, et al: Evaluation of a nurse-led safety program in a critical care unit. J Nurs Care Qual 2013;28:139

246. Sexton J, Sharek P, Thomas E: Exposure to leadership WalkRounds in neonatal intensive care units is associated with a better patient safety culture and less caregiver burnout. BMJ Qual Saf 2014;814

247. Siow E, Wypij D, Berry P, et al: The effect of continuity in nursing care on patient outcomes in the pediatric intensive care unit. $J$ Nurs Adm 2013;43:394-402 
248. Bowman K: Trepidation: A family member's response to shift change in the intensive care unit. Dimens Crit Care Nurs 2010;29:288-292

249. Garcia X, Sachdeva R, Swearingen CJ, et al: A novel paradigm for providing improved care to chronic patients in cardiac intensive care unit. Congenit Heart Dis 2012;7:403-409

250. Kerlin MP, Small DS, Cooney E, et al: A randomized trial of nighttime physician staffing in an intensive care unit. $N$ Engl $J$ Med 2013;368:2201-2209

251. McAdam JL, Dracup KA, White DB, et al: Symptom experiences of family members of intensive care unit patients at high risk for dying. Crit Care Med 2010;38:1078-1085

252. Stremler R, Dhukai Z, Wong L, et al: Factors influencing sleep for parents of critically ill hospitalised children: A qualitative analysis. Intensive Crit Care Nurs 2011;27:37-45

253. Halm MA, Titler MG, Kleiber C, et al: Behavioral responses of family members during critical illness. Clin Nurs Res 1993;2:414437

254. Van Horn E, Tesh A: The effect of critical care hospitalization on family members: Stress and responses. Dimens Crit Care Nurs 2000;19:40-49 
255. Lee SY, Kimble LP: Impaired sleep and well-being in mothers with low-birth-weight infants. JOGNN - Journal of Obstetric, Gynecologic, and Neonatal Nursing 2009;38:676-685

256. Busse M, Stromgren K, Thorngate L, et al: Parents' responses to stress in the neonatal intensive care unit. Crit Care Nurse $2013 ; 33: 52-60$

257. Stremler R, Dhukai Z, Pullenayegum E, et al: Sleep, sleepiness, and fatigue outcomes for parents of critically ill children. Pediatr Crit Care Med 2014;15:e56-65

258. Arora V, Dunphy C, Chang VY, et al: The effects of on-duty napping on intern sleep time and fatigue. Ann Intern Med $2006 ; 144: 792-798$

259. Philip P, Taillard J, Moore N, et al: The effects of coffee and napping on nighttime highway driving: A randomized trial. Ann Intern Med 2006;144:785-791

260. Faraut B, Leger D, Medkour T, et al: Napping reverses increased pain sensitivity due to sleep restriction. PLoS One 2015;10:e0117425

261. COMMITTEE ON HOSPITAL CARE and INSTITUTE FOR PATIENT- AND FAMILY-CENTERED CARE: Patient- and familycentered care and the pediatrician's role. Pediatrics 2012;129:394-404 
262. Committee on Hospital Care. American Academy of Pediatrics: Family-centered care and the pediatrician's role. Pediatrics 2003;112:691-697

263. Beryl Institute.: The state of patient experience in american hospitals 2013: Positive trends and opportunities for the future. Washington DC, The Beryl Institute, 2013

264. Bliss: The bliss baby charter standards, 2009. http://www.bliss.org.uk/order-publications/\#hp Edition. London, Bliss Publications, 2009

265. Bliss: Bliss baby charter audit tool, 2012. http://www.bliss.org.uk/order-publications/\#hp Edition. London, Bliss Publications, 2012

266. Dunn MS, Reilly MC, Johnston AM, et al: Development and dissemination of potentially better practices for the provision of family-centered care in neonatology: The family-centered care map. Pediatrics 2006;118:95-107-95-107

267. Kuo DZ, Houtrow AJ, Arango P, et al: Family-centered care: Current applications and future directions in pediatric health care. Maternal and Child Health Journal, Matern Child Health J 2011;16:297-305

268. Ludwig S, Day S: New standards for resident professionalism: Discussion and justification. Philibert I and Amis S, editors.

Chicago, Accreditation Council for Graduate Medical Education, 2011 
269. Mann KJ, Hoffman A, Miller DJ, et al: The effect of a patient- and family-centered care curriculum on pediatrics residents' patient-centeredness. J Grad Med Educ 2013;5:36-40

270. Ortenstrand A, Westrup B, Brostrom EB, et al: The stockholm neonatal family centered care study: Effects on length of stay and infant morbidity. Pediatrics 2010;125:e278-85

271. Johnson B, Abraham M, Conway J, et al: Partnering with patients and families to design a patient-and family-centered health care system. Bethesda MD: Institute for Family-Centered Care 2008;

272. Earp JAL, French EA, Gilkey MB: Patient advocacy for health care quality: Strategies for achieving patient-centered care. Sudbury, Mass., Jones and Bartlett Pub., 2008

273. Philibert I, Patow C, Cichon J: Incorporating patient- and family-centered care into resident education: Approaches, benefits, and challenges. J Grad Med Educ 2011;3:272-278

274. Schappin R, Wijnroks L, Uniken Venema MM, et al: Rethinking stress in parents of preterm infants: A meta-analysis. PLoS One 2013;8:e54992

275. Stewart M: Towards a global definition of patient centred care. BMJ 2001;322:444-445 
276. Truog RD, Campbell ML, Curtis JR, et al: Recommendations for end-of-life care in the intensive care unit: A consensus statement by the american college [corrected] of critical care medicine. Crit Care Med 2008;36:953-963

277. Institute of Medicine (U.S.). Committee on Quality of Health Care in America: Crossing the quality chasm. Washington, D.C., National Academy Press, 2001

278. Institute for Patient- and Family-Centered Care: What is meant by the word "family"? http://www.ipfcc.org/faq.html Edition. 2014 